\title{
HIGHEST WEIGHT VECTORS OF MIXED TENSOR PRODUCTS OF GENERAL LINEAR LIE SUPERALGEBRAS
}

\author{
HEBING RUI AND YUCAI SU
}

\begin{abstract}
In this paper, a notion of cyclotomic (or level $k$ ) walled Brauer algebras $\mathscr{B}_{k, r, t}$ is introduced for arbitrary positive integer $k$. It is proven that $\mathscr{B}_{k, r, t}$ is free over a commutative ring with rank $k^{r+t}(r+t)$ ! if and only if it is admissible. Using super Schur-Weyl duality between general linear Lie superalgebras $\mathfrak{g l}_{m \mid n}$ and $\mathscr{B}_{2, r, t}$, we give a classification of highest weight vectors of $\mathfrak{g l}_{m \mid n}$-modules $M_{p q}^{r t}$, the tensor products of Kac-modules with mixed tensor products of the natural module and its dual. This enables us to establish an explicit relationship between $\mathfrak{g l}_{m \mid n}$-Kac-modules and right cell (or standard) $\mathscr{B}_{2, r, t}$-modules over $\mathbb{C}$. Further, we find an explicit relationship between indecomposable tilting $\mathfrak{g l}_{m \mid n}$-modules appearing in $M_{p q}^{r t}$, and principal indecomposable right $\mathscr{B}_{2, r, t}$-modules via the notion of Kleshchev bipartitions. As an application, decomposition numbers of $\mathscr{B}_{2, r, t}$ arising from super Schur-Weyl duality are determined.
\end{abstract}

\section{INTRODUCTION}

Motivated by Brundan-Stroppel's work on higher super Schur-Weyl duality in [5], we introduced affine walled Brauer algebras $\mathscr{B}_{r, t}^{\text {aff }}$ in [19] so as to establish higher super Schur-Weyl duality on the tensor product $M_{p q}^{r t}$ of a Kac-module with a mixed tensor product of the natural module and its dual for general linear Lie superalgebra $\mathfrak{g l}_{m \mid n}$ over $\mathbb{C}$ under the assumption $r+t \leq \min \{m, n\} 11$ One of purposes of this paper is to generalize super Schur-Weyl duality to the case $r+t>\min \{m, n\}$. For this aim, we need to establish a bijective map from a level two walled Brauer algebra $\mathscr{B}_{2, r, t}$ appearing in [19] to a level two degenerate Hecke algebra $\mathscr{H}_{2, r+t}$. This can be done by showing that the dimension of $\mathscr{B}_{2, r, t}$ is $2^{r+t}(r+t)$ ! over $\mathbb{C}$. We consider this problem in a general setting by introducing a cyclotomic (or level $k$ ) walled Brauer algebra $\mathscr{B}_{k, r, t}$ for arbitrary $k \in \mathbb{Z}^{>0}$. By employing a totally new method, which is independent of seminormal forms of $\mathscr{B}_{k, r, t}$, we prove that $\mathscr{B}_{k, r, t}$ is free over a commutative ring $R$ with rank $k^{r+t}(r+t)$ ! if and only if it is admissible in the sense of Definition 2.3. It is expected that $\mathscr{B}_{k, r, t}$ can be used to study the problem on a classification of finite dimensional simple $\mathscr{B}_{r, t}^{\text {aff }}$-modules over an algebraically closed field. Details will be given elsewhere.

The establishment of the higher super Schur-Weyl duality [19] enables us to use the representation theory of $\mathscr{B}_{2, r, t}$ to classify highest weight vectors of $M_{p q}^{r t}$ (at this point, we would like to mention that purely on the Lie superalgebra side, it seems to be hard to construct highest weight vectors of a given module, which is an interesting problem on its own right). On the other hand, a classification of highest weight vectors of $M_{p q}^{r t}$ also enables us to relate the category of finite dimensional $\mathfrak{g l}_{m \mid n}$-modules with that of $\mathscr{B}_{2, r, t}$, which in turn gives us

Supported by NSFC (grant no. 11025104, 11371278), Shanghai Municipal Science and Technology Commission 11XD1402200, 12XD1405000, and Fundamental Research Funds for the Central Universities of China.

${ }^{1}$ After we finished [19], Professor Stroppel informed us that Sartori defined affine walled algebras via affine walled Brauer category, independently in [20]. 
an efficient way to calculate decomposition numbers of $\mathscr{B}_{2, r, t}$ (cf. [18] for quantum walled Brauer algebras). This is the main motivation of this paper. We explain some details below.

It is proven in [19] that $\operatorname{End}_{U\left(\mathfrak{g l}_{m \mid n}\right)}\left(M_{p q}^{r t}\right) \cong \mathscr{B}_{2, r, t}$ if $r+t \leq \min \{m, n\}$. Since there is a bijection between the dominant weights of $M_{p q}^{r t}$ and the poset $\Lambda_{2, r, t}$ in (3.12), and since $\mathscr{B}_{2, r, t}$ is a weakly cellular algebra over $\Lambda_{2, r, t}$ in the sense of [11], it is very natural to ask the following problem: whether a $\mathbb{C}$-space of $\mathfrak{g l}_{m \mid n}$-highest weight vectors of $M_{p q}^{r t}$ with a fixed highest weight is isomorphic to a cell (or standard) module of $\mathscr{B}_{2, r, t}$.

We give an affirmative answer to the problem. In sharp contrast to the Lie algebra case, due to the existence of the parity of $\mathfrak{g l}_{m \mid n}$, the known weakly cellular basis of $\mathscr{B}_{2, r, t}$ in [19] can

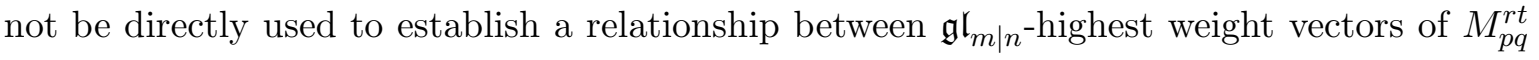
and right cell modules of $\mathscr{B}_{2, r, t}$. One has to find new cellular bases of level two Hecke algebra $\mathscr{H}_{2, r}$ which are different from that in [3]. These new cellular bases of $\mathscr{H}_{2, r}$, which relate both trivial and signed representations of symmetric groups, are used to construct a new weakly cellular basis of $\mathscr{B}_{2, r, t}$. Motivated by explicit descriptions of bases of right cell modules for $\mathscr{B}_{2, r, t}$, we construct and classify $\mathfrak{g l}_{m \mid n}$-highest weight vectors of $M_{p q}^{r t}$. This leads to a $\mathscr{B}_{2, r, t^{-}}$ module isomorphism between each $\mathbb{C}$-space of $\mathfrak{g l}_{m \mid n}$-highest weight vectors of $M_{p q}^{r t}$ with a fixed highest weight and the corresponding cell module of $\mathscr{B}_{2, r, t}$. Based on the above, we are able to construct a suitable exact functor sending $\mathfrak{g l}_{m \mid n}$-Kac-modules to right cell modules of $\mathscr{B}_{2, r, t}$. This functor also sends an indecomposable tilting module appearing in $M_{p q}^{r t}$ to a principal indecomposable right $\mathscr{B}_{2, r, t}$-module indexed by a pair of so-called Kleshchev bipartitions in the sense of (3.15). It gives us an efficient way to calculate decomposition numbers of $\mathscr{B}_{2, r, t}$ via Brundan-Stroppel's result [5] on the multiplicity of a Kac-module in an indecomposable tilting module appearing in $M_{p q}^{r t}$.

We organize the paper as follows. In section 2 , after recalling the definition of $\mathscr{B}_{r, t}^{\text {aff }}$ over a commutative ring $R$, we introduce cyclotomic walled Brauer algebras $\mathscr{B}_{k, r, t}:=\mathscr{B}_{r, t}^{\text {aff }} / I$ for arbitrary $k \in \mathbb{Z}^{>0}$, where $I$ is the two-sided ideal of $\mathscr{B}_{r, t}^{\text {aff }}$ generated by two cyclotomic polynomials $\boldsymbol{f}\left(x_{1}\right)$ and $\boldsymbol{g}\left(\bar{x}_{1}\right)$ of degree $k$, which satisfy (2.5)-(2.7). When $\mathscr{B}_{r, t}^{\text {aff }}$ is admissible in the sense of Definition 2.3, we describe explicitly an $R$-basis of $I$. This enables us to prove that $\mathscr{B}_{k, r, t}$ is free over $R$ with rank $k^{r+t}(r+t)$ ! if and only if it is admissible. In section 3 , we construct cellular bases of $\mathscr{H}_{2, r}$ and use them to construct a weakly cellular

basis of $\mathscr{B}_{2, r, t}$. In section 4, higher super Schur-Weyl dualities in [19] are generalized to the case $r+t>\min \{m, n\}$. In sections $5-6$, we classify highest weight vectors of $M_{p q}^{r 0}$ and $M_{p q}^{r t}$. Based on this, we establish an explicit relationship between indecomposable tilting (resp. Kac) modules for $\mathfrak{g l}_{m \mid n}$ and principal indecomposable (resp. cell) right $\mathscr{B}_{2, r, t}$-modules via a suitable exact functor. This gives us an efficient way to calculate decomposition numbers of $\mathscr{B}_{2, r, t}$ arising from the super Schur-Weyl duality in [19].

\section{Affine walled Brauer algebras and its Cyclotomic quotients}

Throughout, we assume that $R$ is a commutative ring containing $\Omega=\left\{\omega_{a} \mid a \in \mathbb{N}\right\}$ and identity 1 . In this section, we introduce a level $k$ walled Brauer algebra $\mathscr{B}_{k, r, t}$ and prove that 
$\mathscr{B}_{k, r, t}$ is free over $R$ with rank $k^{r+t}(r+t)$ ! if and only if $\mathscr{B}_{k, r, t}$ is admissible in the sense of Definition 2.3. First, we briefly recall the definition of walled Brauer algebras.

Fix $r, t \in \mathbb{Z}^{>0}$. A walled ( $r, t$ )-Brauer diagram (or simply, a walled Brauer diagram) is a diagram with $(r+t)$ vertices on top and bottom rows, and vertices on both rows are labeled from left to right by $r, \ldots, 2,1, \overline{1}, \overline{2}, \ldots, \bar{t}$, such that every $i \in\{r, \ldots, 2,1\}$ (resp., $\bar{i} \in\{\overline{1}, \overline{2}, \ldots, \bar{t}\}$ ) on each row is connected to a unique $\bar{j}$ (resp., $j$ ) on the same row or a unique $j$ (resp., $\bar{j}$ ) on the other row. Thus there are four types of pairs $[i, j],[i, \bar{j}],[\bar{i}, j]$ and $[\bar{i}, \bar{j}]$. The pairs $[i, j]$ and $[\bar{i}, \bar{j}]$ are vertical edges, and $[\bar{i}, j]$ and $[i, \bar{j}]$ are horizontal edges.

The product of two walled Brauer diagrams $D_{1}$ and $D_{2}$ can be defined via concatenation. Putting $D_{1}$ above $D_{2}$ and connecting each vertex on the bottom row of $D_{1}$ to the corresponding vertex on the top row of $D_{2}$ yields a diagram $D_{1} \circ D_{2}$, called the concatenation of $D_{1}$ and $D_{2}$. Removing all circles of $D_{1} \circ D_{2}$ yields a unique walled Brauer diagram, denoted $D_{3}$. Let $n$ be the number of circles appearing in $D_{1} \circ D_{2}$. Then the product $D_{1} D_{2}$ is defined to be $\omega_{0}^{n} D_{3}$, where $\omega_{0}$ is a fixed element in $R$. The walled Brauer algebra [16, 23, 17] $\mathscr{B}_{r, t}:=\mathscr{B}_{r, t}\left(\omega_{0}\right)$ with defining parameter $\omega_{0}$ is the associative $R$-algebra spanned by all walled Brauer diagrams with product defined in this way.

Let $\mathfrak{S}_{r}\left(\right.$ resp. $\left.\overline{\mathfrak{S}}_{t}\right)$ be the symmetric group in $r$ (resp. $t$ ) letters $r, \ldots, 2,1$ (resp. $\overline{1}, \overline{2}, \ldots, \bar{t}$ ). It is known that $\mathscr{B}_{r, t}$ contains two subalgebras which are isomorphic to the group algebras of $\mathfrak{S}_{r}$ and $\overline{\mathfrak{S}}_{t}$, respectively. More explicitly, the walled Brauer diagram $s_{i}$ whose edges are of forms $[k, k]$ and $[\bar{k}, \bar{k}]$ except two vertical edges $[i, i+1]$ and $[i+1, i]$ can be identified with the basic transposition $(i, i+1) \in \mathfrak{S}_{r}$, which switches $i$ and $i+1$ and fixes others. Similarly, there is a walled Brauer diagram $\bar{s}_{j}$ corresponding to $(\bar{j}, \overline{j+1}) \in \overline{\mathfrak{S}}_{t}$. Let $e_{1}$ be the walled Brauer diagram whose edges are of forms $[k, k]$ and $[\bar{k}, \bar{k}]$ except two horizontal edges $[1, \overline{1}]$ on the top and bottom rows. Then $\mathscr{B}_{r, t}$ is the $R$-algebra [17] generated by $e_{1}, s_{i}, \bar{s}_{j}$ for $1 \leq i \leq r-1,1 \leq j \leq t-1$ such that $s_{i}$ 's, $\bar{s}_{j}$ 's are distinguished generators of $\mathfrak{S}_{r} \times \overline{\mathfrak{S}}_{t}$ and

$$
\begin{array}{ll}
e_{1}^{2}=\omega_{0} e_{1}, \quad e_{1} s_{1} e_{1}=e_{1}=e_{1} \bar{s}_{1} e_{1}, & s_{i} e_{1}=e_{1} s_{i}, \bar{s}_{j} e_{1}=e_{1} \bar{s}_{j} \quad(i, j \neq 1), \\
e_{1} s_{1} \bar{s}_{1} e_{1} s_{1}=e_{1} s_{1} \bar{s}_{1} e_{1} \bar{s}_{1}, & s_{1} e_{1} s_{1} \bar{s}_{1} e_{1}=\bar{s}_{1} e_{1} s_{1} \bar{s}_{1} e_{1} .
\end{array}
$$

Let $\mathscr{H}_{n}^{\text {aff }}$ be the degenerate affine Hecke algebra [10]. As a free R-module, it is the tensor product $R\left[y_{1}, y_{2}, \cdots, y_{n}\right] \otimes R \mathfrak{S}_{n}$ of a polynomial algebra with the group algebra of $\mathfrak{S}_{n}$. The multiplication is defined so that $R\left[y_{1}, y_{2}, \cdots, y_{n}\right] \equiv R\left[y_{1}, y_{2}, \cdots, y_{n}\right] \otimes 1$ and $R \mathfrak{S}_{n} \equiv 1 \otimes R \mathfrak{S}_{n}$ are subalgebras and $s_{i} y_{j}=y_{j} s_{i}$ if $j \neq i, i+1$ and $s_{i} y_{i}=y_{i+1} s_{i}-1,1 \leq i \leq n-1$.

Recall that $R$ contains 1 and $\boldsymbol{\Omega}=\left\{\omega_{a} \in R \mid a \in \mathbb{N}\right\}$. The affine walled Brauer algebra $\mathscr{B}_{r, t}^{\text {aff }}(\boldsymbol{\Omega})$ (which is $\widehat{\mathscr{B}_{r, t}}$ in $[19, \S 4]$ ) with respect to the defining parameters $\omega_{a}$ 's have been defined via generators and 26 defining relations [19, Definition 2.7]. It follows from [19, Theorem 4.15] that $\mathscr{B}_{r, t}^{\text {aff }}(\boldsymbol{\Omega})$ can be also defined in a simpler way as follows: it is an associative $R$-algebra generated by $e_{1}, x_{1}, \bar{x}_{1}, s_{i}, \bar{s}_{j}$ for $1 \leq i \leq r-1,1 \leq j \leq t-1$, such that $e_{1}, s_{i}$ 's, $\bar{s}_{j}$ 's are generators of $\mathscr{B}_{r, t}$ with defining parameter $\omega_{0}$, and as a free $R$-module,

$$
\mathscr{B}_{r, t}^{\mathrm{aff}}(\boldsymbol{\Omega})=R\left[\mathbf{x}_{r}\right] \otimes \mathscr{B}_{r, t} \otimes R\left[\overline{\mathbf{x}}_{t}\right],
$$

the tensor product of the walled Brauer algebra $\mathscr{B}_{r, t}$ with two polynomial algebras

$$
R\left[\mathbf{x}_{r}\right]:=R\left[x_{1}, x_{2}, \cdots, x_{r}\right], \text { and } R\left[\overline{\mathbf{x}}_{t}\right]:=R\left[\bar{x}_{1}, \bar{x}_{2}, \cdots, \bar{x}_{t}\right] .
$$


Multiplication is defined as $R\left[\mathbf{x}_{r}\right] \equiv R\left[\mathbf{x}_{r}\right] \otimes 1 \otimes 1, R\left[\overline{\mathbf{x}}_{r}\right] \equiv 1 \otimes 1 \otimes R\left[\overline{\mathbf{x}}_{r}\right]$, and $\mathscr{B}_{r, t} \equiv 1 \otimes \mathscr{B}_{r, t} \otimes 1$, and $R\left[\mathbf{x}_{r}\right] \otimes R \mathfrak{S}_{r} \otimes 1 \cong \mathscr{H}_{r}^{\text {aff }} \otimes 1,1 \otimes R \overline{\mathfrak{S}}_{t} \otimes R\left[\overline{\mathbf{x}}_{r}\right] \cong 1 \otimes \mathscr{H}_{t}^{\text {aff }}$ and

$$
\begin{aligned}
& e_{1}\left(x_{1}+\bar{x}_{1}\right)=\left(x_{1}+\bar{x}_{1}\right) e_{1}=0, \quad s_{1} e_{1} s_{1} x_{1}=x_{1} s_{1} e_{1} s_{1}, \quad \bar{s}_{1} e_{1} \bar{s}_{1} \bar{x}_{1}=\bar{x}_{1} \bar{s}_{1} e_{1} \bar{s}_{1}, \\
& s_{i} \bar{x}_{1}=\bar{x}_{1} s_{i}, \quad \bar{s}_{i} x_{1}=x_{1} \bar{s}_{i}, \quad x_{1}\left(e_{1}+\bar{x}_{1}\right)=\left(e_{1}+\bar{x}_{1}\right) x_{1}, \\
& e_{1} x_{1}^{k} e_{1}=\omega_{k} e_{1}, \quad e_{1} \bar{x}_{1}^{k} e_{1}=\bar{\omega}_{k} e_{1}, \quad \forall k \in \mathbb{Z}^{\geq 0},
\end{aligned}
$$

where $\bar{\omega}_{a}$ 's are determined by [19, Corollary 4.3]. If $\bar{\omega}_{a}$ 's do not satisfy [19, Corollary 4.3], then $e_{1}=0$ and $\mathscr{B}_{r, t}^{\text {aff }}(\boldsymbol{\Omega})$ turns out to be $\mathscr{H}_{r}^{\text {aff }} \otimes \mathscr{H}_{t}^{\text {aff }}$ if $R$ is a field.

We remark that the isomorphism $R\left[\mathbf{x}_{r}\right] \otimes R \mathfrak{S}_{r} \otimes 1 \cong \mathscr{H}_{r}^{\text {aff }} \otimes 1$ sends $s_{i}$ 's (resp. $x_{1}$ ) to $s_{i}$ 's (resp. $-y_{1}$ ), and the isomorphism $1 \otimes R \overline{\mathfrak{S}}_{t} \otimes R\left[\overline{\mathbf{x}}_{r}\right] \cong 1 \otimes \mathscr{H}_{t}^{\text {aff }}$ sends $\bar{s}_{j}$ 's (resp. $\bar{x}_{1}$ ) to $s_{j}$ 's $\left(\right.$ resp. $\left.-y_{1}\right)$. So, $x_{i+1}=s_{i} x_{i} s_{i}-s_{i}$ and $\bar{x}_{j+1}=\bar{s}_{j} \bar{x}_{j} \bar{s}_{j}-\bar{s}_{j}$ and $y_{i+1}=s_{i} y_{i} s_{i}+s_{i}$ if all of them make sense.

For the simplification of notation, we denote $\mathscr{B}_{r, t}^{\text {aff }}(\boldsymbol{\Omega})$ by $\mathscr{B}_{r, t}^{\text {aff }}$. Fix $u_{1}, u_{2}, \cdots, u_{k} \in R$ for some $k \in \mathbb{Z}^{>0}$. Let $\boldsymbol{f}\left(x_{1}\right) \in \mathscr{B}_{r, t}^{\text {aff }}$ be such that

$$
\boldsymbol{f}\left(x_{1}\right)=\prod_{i=1}^{k}\left(x_{1}-u_{i}\right) .
$$

By [19, Lemma 4.2] (or using (2.2) $-(2.3)$ ), there is a monic polynomial $\boldsymbol{g}\left(\bar{x}_{1}\right) \in R\left[\bar{x}_{1}\right]$ with degree $k$ such that

$$
e_{1} \boldsymbol{f}\left(x_{1}\right)=(-1)^{k} e_{1} \boldsymbol{g}\left(\bar{x}_{1}\right)
$$

If $R$ is an algebraically closed field, then there are $\bar{u}_{1}, \bar{u}_{2}, \cdots, \bar{u}_{k} \in R$ such that

$$
\boldsymbol{g}\left(\bar{x}_{1}\right)=\prod_{i=1}^{k}\left(\bar{x}_{1}-\bar{u}_{i}\right)
$$

Definition 2.1. Let $R$ be a commutative ring containing $1, \Omega=\left\{\omega_{a} \in R \mid a \in \mathbb{N}\right\}$, and $u_{i}, \bar{u}_{i}, 1 \leq i \leq k$. The cyclotomic (or level $k$ ) walled Brauer algebra $\mathscr{B}_{k, r, t}$ is the quotient algebra $\mathscr{B}_{r, t}^{\text {aff }} / I$, where $I$ is the two-sided ideal of $\mathscr{B}_{r, t}^{\text {aff }}$ generated by $\boldsymbol{f}\left(x_{1}\right)$ and $\boldsymbol{g}\left(\bar{x}_{1}\right)$ satisfying (2.5)-(2.7).

If $k=1$, then $\mathscr{B}_{k, r, t}$ is $\mathscr{B}_{r, t}$ with defining parameter $\omega_{0}$. For some special $u_{i}, \bar{u}_{i}, i=1,2$, $\mathscr{B}_{2, r, t}$ is the level two walled Brauer algebras arising from super Schur-Weyl duality in [19].

Lemma 2.2. Let $\boldsymbol{f}\left(x_{1}\right)$ be given in (2.5). Write $\boldsymbol{f}\left(x_{1}\right)=x_{1}^{k}+\sum_{i=1}^{k} a_{i} x_{1}^{k-i}$. Then $e_{1}$ is an $R$-torsion element of $\mathscr{B}_{k, r, t}$ unless

$$
\omega_{\ell}=-\left(a_{1} \omega_{\ell-1}+\cdots a_{k} \omega_{\ell-k}\right) \text { for all } \ell \geq k .
$$

Proof. Let $b_{\ell}=\omega_{\ell}+a_{1} \omega_{\ell-1}+\cdots+a_{k} \omega_{\ell-k} \in R$. By (2.4),$b_{\ell} e_{1}=e_{1} f\left(x_{1}\right) x_{1}^{\ell-k} e_{1}$ in $\mathscr{B}_{r, t}^{\text {aff }}$ and $b_{\ell} e_{1}=0$ in $\mathscr{B}_{k, r, t}$. Thus, $e_{1}$ is an $R$-torsion element if $b_{\ell} \neq 0$ for some $\ell \geq k$.

Definition 2.3. The algebras $\mathscr{B}_{r, t}^{\text {aff }}$ and $\mathscr{B}_{k, r, t}$ are called admissible if (2.8) holds.

Lemma 2.4. Assume $\boldsymbol{f}\left(x_{1}\right), \boldsymbol{g}\left(\bar{x}_{1}\right) \in \mathscr{B}_{r, t}^{\mathrm{aff}}$ satisfying (2.5)-(2.7) . If $\mathscr{B}_{r, t}^{\mathrm{aff}}$ is admissible, then

(1) $e_{1} f\left(x_{1}\right) x_{1}^{a} e_{1}=0$ for all $a \in \mathbb{N}$.

(2) $e_{1} \boldsymbol{g}\left(\bar{x}_{1}\right) \bar{x}_{1}^{a} e_{1}=0$ for all $a \in \mathbb{N}$. 
Proof. (1) trivial since $\mathscr{B}_{r, t}^{\text {aff }}$ is admissible. It is proven in [19] that there is an $R$-linear antiinvolution $\sigma$ on $\mathscr{B}_{r, t}^{\text {aff }}$, which fixes all generators of $\mathscr{B}_{r, t}^{\text {aff }}$. Applying $\sigma$ on [19, Lemma 4.2] yields

$$
\bar{x}_{1}^{k} e_{1}=\sum_{i=0}^{k} a_{k, i} x_{1}^{i} e_{1}, \text { for some } a_{k, i} \in R .
$$

So, (2) follows from (2.6) and (1), immediately.

Denote $s_{i, j}=s_{i} s_{i+1} \cdots s_{j-1}$ if $i<j$, and 1 if $i=j$, and $s_{i-1} s_{i-2} \cdots s_{j}$ if $i>j$. Denote $\bar{s}_{i, j} \in \overline{\mathfrak{S}}_{t}$ similarly. Let $e_{i, j}$ be the walled Brauer diagram such that each vertical edge of $e_{i, j}$ is of form $[k, k]$ or $[\bar{k}, \bar{k}]$ and the horizontal edges on the top and bottom rows of $e_{i, j}$ are $[i, \bar{j}]$. Then

$$
e_{i, j}=\bar{s}_{j, 1} s_{i, 1} e_{1} s_{1, i} \bar{s}_{1, j} \text { for } i, j \text { with } 1 \leq i \leq r \text { and } 1 \leq j \leq t .
$$

For each nonnegative integer $f \leq \min \{r, t\}$, let

$$
e^{f}=e_{1} e_{2} \cdots e_{f} \text { for } f>0 \text { and } e^{0}=1 \text {, where } e_{i}=e_{i, i} .
$$

Set

$$
\mathscr{D}_{r, t}^{f}=\left\{s_{f, i_{f}} \bar{s}_{f, j_{f}} \cdots s_{1, i_{1}} \bar{s}_{1, j_{1}} \mid 1 \leq i_{1}<\cdots<i_{f} \leq r, k \leq j_{k}\right\} .
$$

Definition 2.5. For $\alpha=\left(\alpha_{1}, \cdots, \alpha_{r}\right) \in \mathbb{N}^{r}$ and $\beta=\left(\beta_{1}, \cdots, \beta_{t}\right) \in \mathbb{N}^{t}$, let $x^{\alpha}=\prod_{i=1}^{r} x_{i}^{\alpha_{i}}$, $\bar{x}^{\beta}=\prod_{j=1}^{t} \bar{x}_{j}^{\beta_{j}}$. Let $\mathcal{M}$ be a subset of $\mathscr{B}_{r, t}^{\text {aff }}$ given by

$$
\mathcal{M}=\bigcup_{f=0}^{\min \{m, n\}}\left\{x^{\alpha} c^{-1} e^{f} w d \bar{x}^{\beta} \mid(\alpha, \beta) \in \mathbb{N}^{r} \times \mathbb{N}^{t}, c, d \in \mathscr{D}_{r, t}^{f}, w \in \mathfrak{S}_{r-f} \times \overline{\mathfrak{S}}_{t-f}\right\} .
$$

Elements of $\mathcal{M}$ are called regular monomials of $\mathscr{B}_{r, t}^{\text {aff }}$.

Theorem 2.6. [19, Theorem 4.15] The affine walled Brauer algebra $\mathscr{B}_{r, t}^{\mathrm{aff}}$ is free over $R$ with $\mathcal{M}$ as its $R$-basis.

We consider $\mathscr{B}_{r, t}^{\text {aff }}$ as a filtrated $R$-algebra as follows. Let

$$
\operatorname{deg} s_{i}=\operatorname{deg} \bar{s}_{j}=\operatorname{deg} e_{1}=0 \text { and } \operatorname{deg} x_{k}=\operatorname{deg} \bar{x}_{\ell}=1
$$

for all possible $i, j, k, \ell$ 's. Let $\left(\mathscr{B}_{r, t}^{\text {aff }}\right)^{(k)}$ be the $R$-submodule spanned by regular monomials with degrees less than or equal to $k$ for $k \in \mathbb{Z}^{\geq 0}$. Then we have the following filtration

$$
\mathscr{B}_{r, t}^{\text {aff }} \supset \cdots \supset\left(\mathscr{B}_{r, t}^{\text {aff }}\right)^{(1)} \supset\left(\mathscr{B}_{r, t}^{\text {aff }}\right)^{(0)} \supset\left(\mathscr{B}_{r, t}^{\text {aff }}\right)^{(-1)}=0 .
$$

Let $\operatorname{gr}\left(\mathscr{B}_{r, t}^{\text {aff }}\right)=\oplus_{i \geq 0}\left(\mathscr{B}_{r, t}^{\text {aff }}\right)^{[i]}$, where $\left(\mathscr{B}_{r, t}^{\text {aff }}\right)^{[i]}=\left(\mathscr{B}_{r, t}^{\text {aff }}\right)^{(i)} /\left(\mathscr{B}_{r, t}^{\text {aff }}\right)^{(i-1)}$. Then $\operatorname{gr}\left(\mathscr{B}_{r, t}^{\text {aff }}\right)$ is an associated $\mathbb{Z}$-graded algebra. We will use the same symbols to denote elements in $\operatorname{gr}\left(\mathscr{B}_{r, t}^{\text {aff }}\right)$.

Lemma 2.7. Let $x_{i}^{\prime}=s_{i-1} x_{i-1}^{\prime} s_{i-1}$, and $\bar{x}_{j}^{\prime}=\bar{s}_{j-1} \bar{x}_{j-1} \bar{s}_{j-1}$ for $i, j \in \mathbb{Z} \geq 2$ with $i \leq r$ and $j \leq t$, where $x_{1}^{\prime}=x_{1}$, and $\bar{x}_{1}^{\prime}=\bar{x}_{1}$.

(1) $x_{i}=x_{i}^{\prime}-L_{i}$, where $L_{i}=\sum_{1 \leq j<i}(j, i)$ and $(j, i)$ is the transposition in $\mathfrak{S}_{r}$ which switches $j, i$ and fixes others.

(2) $\bar{x}_{i}=\bar{x}_{i}^{\prime}-\bar{L}_{i}$, where $\bar{L}_{i}=\sum_{\overline{1} \leq \bar{j}<\bar{i}}(\bar{j}, \bar{i})$ and $(\bar{j}, \bar{i})$ is the transposition in $\overline{\mathfrak{S}}_{t}$ which switches $\bar{j}, \bar{i}$ and fixes others.

(3) Any symmetric polynomial of $L_{1}, L_{2}, \cdots, L_{r}$ (resp. $\left.\bar{L}_{1}, \bar{L}_{2}, \cdots, \bar{L}_{t}\right)$ is a central element of $R \mathfrak{S}_{r}\left(\right.$ resp. $\left.R \overline{\mathfrak{S}}_{\mathfrak{t}}\right)$. 
Proof. (1)-(2) are trivial and (3) is a well-known result.

The elements $L_{i}$ 's (resp. $\bar{L}_{j}$ 's) are known as Jucys-Murphy elements of $R \mathfrak{S}_{r}$ (resp. $R \overline{\mathfrak{S}}_{\mathfrak{t}}$ ). Note that $x_{i} x_{j}=x_{j} x_{i}$ and $\bar{x}_{i} \bar{x}_{j}=\bar{x}_{j} \bar{x}_{i}$ for all possible $i, j$. However, $x_{i}^{\prime}$ and $x_{j}^{\prime}$ (resp. $\bar{x}_{i}^{\prime}$ and $\bar{x}_{j}^{\prime}$ ) do not commute each other.

Suppose $0<f \leq \min \{m, n\}$. Denote

$$
\vec{i}=\left(i_{1}, . ., i_{f}\right), \quad \vec{j}=\left(j_{1}, \ldots, j_{f}\right), \quad e_{\vec{i}, \vec{j}}=e_{i_{1}, j_{1}} e_{i_{2}, j_{2}} \cdots e_{i_{f}, j_{f}},
$$

where $i_{1}, i_{2}, \cdots, i_{f}$ are distinct numbers in $\{1,2, \cdots, r\}$, and $j_{1}, j_{2}, \cdots, j_{f}$ are distinct numbers in $\{\overline{1}, \overline{2}, \cdots, \bar{t}\}$. Then $e_{i_{k}, j_{k}}$ 's commute each other. If $f=0$, we set $\vec{i}=\vec{j}=\emptyset$ and $e_{\vec{i}, \vec{j}}=1$.

We always assume that $\mathfrak{S}_{r}\left(\operatorname{resp} . \overline{\mathfrak{S}}_{t}\right)$ acts on the right of $\{r, \ldots, 2,1\}$ (resp. $\left.\{\overline{1}, \overline{2}, \ldots, \bar{t}\}\right)$.

Lemma 2.8. Suppose $a \in \mathbb{Z}^{>0}, 1 \leq i, \ell \leq r$ and $1 \leq j \leq t$.

(1) If $w \in \mathfrak{S}_{r}$, then $w \boldsymbol{f}\left(x_{i}^{\prime}\right) w^{-1}=\boldsymbol{f}\left(x_{(i) w^{-1}}^{\prime}\right)$.

(2) If $w \in \overline{\mathfrak{S}}_{t}$, then $\boldsymbol{w} \boldsymbol{g}\left(\bar{x}_{\bar{j}}^{\prime}\right) w^{-1}=\boldsymbol{g}\left(\bar{x}_{(\bar{j}) w^{-1}}^{\prime}\right)$.

(3) $x_{i}^{\prime a} \boldsymbol{f}\left(x_{\ell}^{\prime}\right)=\boldsymbol{f}\left(x_{\ell}^{\prime}\right) x_{i}^{\prime a}+v$, where $v \in \sum_{b<a} \sum_{h, h_{1}=1}^{\max \{i, \ell\}} \boldsymbol{f}\left(x_{h}^{\prime}\right) x_{h_{1}}^{\prime b} R \mathfrak{S}_{r}$.

(4) $\bar{x}_{j}^{\prime a} \boldsymbol{f}\left(x_{i}^{\prime}\right)=\boldsymbol{f}\left(x_{i}^{\prime}\right) \bar{x}_{j}^{\prime a}+v$, where $v \in \sum_{b_{1}+b_{2}<a, c_{1}+c_{2} \leq 1} \epsilon \bar{x}_{j}^{\prime b_{1}} e_{i j}^{c_{1}} \boldsymbol{f}\left(x_{i}^{\prime}\right) e_{i j}^{c_{2}} \bar{x}_{j}^{\prime b_{2}}$ for some nonnegative integers $b_{1}, b_{2}, c_{1}, c_{2}$ and $\epsilon= \pm 1$.

Proof. (1)-(2) are trivial. Since $x_{2}=x_{2}^{\prime}-s_{1}$ and $x_{2} x_{1}=x_{1} x_{2}$,

$$
x_{2}^{\prime} \boldsymbol{f}\left(x_{1}\right)=\boldsymbol{f}\left(x_{1}\right)\left(x_{2}^{\prime}-s_{1}\right)+\boldsymbol{f}\left(x_{2}^{\prime}\right) s_{1} .
$$

Applying the conjugate of $s_{i, 2}$ on (2.15) yields (3) for $a=1$ and $\ell=1$. If $\ell>1$, then $x_{i}^{\prime} f\left(x_{\ell}^{\prime}\right)=x_{i}^{\prime} s_{\ell-1} f\left(x_{\ell-1}^{\prime}\right) s_{\ell-1}=s_{\ell-1} x_{(i) s_{\ell-1}}^{\prime} f\left(x_{\ell-1}^{\prime}\right) s_{\ell-1}$. Thus, (3) follows from inductive assumption on $\ell-1$ and (1) under the assumption $a=1$. The case $a>1$ follows by using the previous result on $a=1$, repeatedly. Finally, (4) can be checked similarly by induction. We leave the details to the readers.

Proposition 2.9. Let $J_{L}=\sum_{i=1}^{t} \mathscr{B}_{r, t}^{\text {aff }} \boldsymbol{g}\left(\bar{x}_{j}^{\prime}\right)$ and $J_{R}=\sum_{i=1}^{r} \boldsymbol{f}\left(x_{i}^{\prime}\right) \mathscr{B}_{r, t}^{\text {aff }}$. Then

(1) $J_{L}$ is a right $R \mathfrak{S}_{r} \otimes \mathscr{H}_{t}^{\text {aff }}-$ module;

(2) $J_{R}$ is a left $\mathscr{H}_{r}^{\text {aff }} \otimes R \overline{\mathfrak{S}}_{t}$-module;

(3) if $\mathscr{B}_{r, t}^{\text {aff }}$ is admissible, then $I=J_{L}+J_{R}$, where $I$ is the two-sided ideal of $\mathscr{B}_{r, t}^{\text {aff }}$ generated by $\boldsymbol{f}\left(x_{1}\right)$ and $\boldsymbol{g}\left(\bar{x}_{1}\right)$ satisfying (2.5) $-(2.7)$.

Proof. Obviously, both $J_{L}$ and $J_{R}$ are $\mathfrak{S}_{r} \times \mathfrak{S}_{\bar{t}}$-bimodules. By Lemma $2.8(3), x_{1} J_{R} \subseteq J_{R}$. Similarly, $J_{L} \bar{x}_{1} \subseteq J_{L}$. This proves (1)-(2). In order to prove (3), it suffices to verify that $J_{L}+J_{R}$ is a two-sided ideal of $\mathscr{B}_{r, t}^{\text {aff }}$. If so, since $\left\{\boldsymbol{f}\left(x_{1}\right), \boldsymbol{g}\left(\bar{x}_{1}\right)\right\} \subset J_{L}+J_{R}, I=J_{L}+J_{R}$, proving the result.

We claim that $e_{1}\left(J_{L}+J_{R}\right) \subseteq J_{L}+J_{R}$ and $\left(J_{L}+J_{R}\right) e_{1} \subseteq J_{L}+J_{R}$. If so, by (2.3), $\left(\bar{x}_{1}+e_{1}\right) \boldsymbol{f}\left(x_{1}\right)=\boldsymbol{f}\left(x_{1}\right)\left(\bar{x}_{1}+e_{1}\right)$ and hence $\bar{x}_{1} \boldsymbol{f}\left(x_{1}\right) \in J_{L}+J_{R}$. By (1)-(2), $\bar{x}_{1} \boldsymbol{f}\left(x_{i}^{\prime}\right)=s_{i, 1} \bar{x}_{1} \boldsymbol{f}\left(x_{1}\right) s_{1, i} \in J_{L}+J_{R}$, and hence $\bar{x}_{1}\left(J_{L}+J_{R}\right) \subseteq J_{L}+J_{R}$. Similarly, $\left(J_{L}+J_{R}\right) x_{1} \subseteq J_{L}+J_{R}$. Thus the claim implies that $J_{L}+J_{R}$ is a two-sided ideal of $\mathscr{B}_{r, t}^{\text {aff }}$. 
By symmetry, it remains to prove $e_{1}\left(J_{L}+J_{R}\right) \subseteq J_{L}+J_{R}$. Obviously, it suffices to verify

$$
e_{1} J_{R} \subset J_{L}+J_{R}
$$

By (2.2),$e_{1} \boldsymbol{f}\left(x_{i}^{\prime}\right)=\boldsymbol{f}\left(x_{i}^{\prime}\right) e_{1}$ for $i \geq 2$. Let $\mathbf{m}$ be a regular monomial of $\mathscr{B}_{r, t}^{\text {aff }}$ defined in (2.12). Then $\mathbf{m}=x^{\alpha} e_{\vec{i}, \vec{j}} w \bar{x}^{\beta}$ for some $w \in \mathfrak{S}_{r} \times \overline{\mathfrak{S}}_{t},(\alpha, \beta) \in \mathbb{N}^{r} \times \mathbb{N}^{t}$ and some $\vec{i}, \vec{j}$. Using induction on $|\alpha|$, we want to prove

$$
e_{1} \boldsymbol{f}\left(x_{1}\right) \mathbf{m} \in J_{L}+J_{R}
$$

If so, then $e_{1} \boldsymbol{f}\left(x_{1}\right) \mathscr{B}_{r, t}^{\text {aff }} \subset J_{L}+J_{R}$ and hence (2.16) follows.

Case 1: $|\alpha|=0$.

If $f=0$, then (2.17) follows from (1) and (2.6). Suppose $1 \leq f \leq \min \{r, t\}$. Since $\mathscr{B}_{r, t}^{\text {aff }}$ is admissible, $e_{1} \boldsymbol{f}\left(x_{1}\right) \mathbf{m}=0$ if $e_{i}$ is a factor of $e_{\vec{i}, \vec{j}}$. Assume that $e_{1}$ is not a factor of $e_{\vec{i}, \vec{j}}$. If there is an $l$ such that $i_{l}=p \neq 1$ and $j_{l}=1$, by (2),

$$
e_{1} \boldsymbol{f}\left(x_{1}\right) e_{p, 1}=s_{p, 2} e_{1} \boldsymbol{f}\left(x_{1}\right) s_{1} e_{1} s_{1, p}=s_{p, 2} e_{1} s_{1} \boldsymbol{f}\left(x_{2}^{\prime}\right) e_{1} s_{1, p}=s_{p, 2} \boldsymbol{f}\left(x_{2}^{\prime}\right) e_{1} s_{1, p} \in J_{R}
$$

Suppose $j_{l} \neq 1$ for all possible $l$. If there is an $l$ such that $e_{i_{l}, j_{l}}=e_{1, p}$ for some $p \neq 1$, then we assume $i_{1}=1$ and $j_{1}=p$ without loss of any generality. In this case,

$$
e_{1} \boldsymbol{f}\left(x_{1}\right) e_{1, p}=(-1)^{k} \bar{s}_{p, 2} e_{1} \boldsymbol{g}\left(\bar{x}_{1}\right) \bar{s}_{1} e_{1} \bar{s}_{1, p}=(-1)^{k} \bar{s}_{p, 2} e_{1} \boldsymbol{g}\left(\bar{x}_{2}^{\prime}\right) \bar{s}_{1, p}=(-1)^{k} \bar{s}_{p, 2} e_{1} \bar{s}_{1, p} \boldsymbol{g}\left(\bar{x}_{1}\right) .
$$

Since $j_{l} \neq 1$ for $1 \leq l \leq f$, by [19, Lemma 4.7(2)], $\bar{x}_{1} e_{i_{l}, j_{l}}=e_{i_{l}, j_{l}} \bar{x}_{1}$ and hence

$$
\boldsymbol{g}\left(\bar{x}_{1}\right) \prod_{l=2}^{f} e_{i_{l}, j_{l}}=\prod_{l=2}^{f} e_{i_{l}, j_{l}} \boldsymbol{g}\left(\bar{x}_{1}\right) \in J_{L} .
$$

Now, (2.17) follows from (1). Finally, if $\left\{i_{l}, j_{l}\right\} \cap\{1\}=\emptyset$ for all possible $l$, then (2.17) follows from (1) and the following fact

$$
e_{1} \boldsymbol{f}\left(x_{1}\right) \prod_{l=1}^{f} e_{i_{f}, j_{f}}=\prod_{l=1}^{f} e_{i_{f}, j_{f}} e_{1} \boldsymbol{f}\left(x_{1}\right)=(-1)^{k} \prod_{l=1}^{f} e_{i_{f}, j_{f}} e_{1} \boldsymbol{g}\left(\bar{x}_{1}\right) \in J_{L} .
$$

Case 2: $|\alpha|>0$.

If $\alpha_{i} \neq 0$ for some $2 \leq i \leq r$, then $e_{1} x_{i}=x_{i}^{\prime} e_{1}-e_{1} \sum_{j=1}^{i}(j, i)$ and $x_{i} \boldsymbol{f}\left(x_{1}\right)=\boldsymbol{f}\left(x_{1}\right) x_{i}$. Let $\mathbf{m}^{\prime}$ be obtained from $\mathbf{m}$ by removing $x_{i}$. Then

$$
e_{1}(1, i) \boldsymbol{f}\left(x_{1}\right) \mathbf{m}^{\prime}=e_{1} \boldsymbol{f}\left(x_{i}^{\prime}\right)(1, i) \mathbf{m}^{\prime}=\boldsymbol{f}\left(x_{i}^{\prime}\right) e_{1}(1, i) \mathbf{m}^{\prime} \in J_{R}
$$

Now, (2.17) follows from inductive assumption on $|\alpha|$. If $\alpha_{i}=0,2 \leq i \leq r$, then $x^{\alpha}=x_{1}^{\alpha_{1}}$ with $\alpha_{1}>0$. Let $v=e_{1} \boldsymbol{f}\left(x_{1}\right) \mathbf{m}$. If $j_{\ell} \neq 1,1 \leq \ell \leq f$, then by (2.18), Lemma 2.8 and inductive assumption,

$$
\begin{aligned}
v & =e_{1} \boldsymbol{f}\left(x_{1}\right) x_{1}^{\alpha_{1}} e_{\vec{i}, \vec{j}} w \bar{x}^{\beta}=(-1)^{k} e_{1} \boldsymbol{g}\left(\bar{x}_{1}\right) x_{1}^{\alpha_{1}} e_{\vec{i}, \vec{j}} w \bar{x}^{\beta} \equiv(-1)^{k} e_{1} x_{1}^{\alpha_{1}} \boldsymbol{g}\left(\bar{x}_{1}\right) e_{\vec{i}, \vec{j}} w \bar{x}^{\beta} \\
& =(-1)^{k} e_{1} x_{1}^{\alpha_{1}} e_{\vec{i}, \vec{j}} \boldsymbol{g}\left(\bar{x}_{1}\right) w \bar{x}^{\beta} \in J_{L} w \bar{x}^{\beta} \subset J_{L}+J_{R},
\end{aligned}
$$

where the " $\equiv$ " is modulo $J_{L}+J_{R}$. Finally, if $j_{\ell}=1$ for some $\ell$, without loss of any generality, we assume $j_{1}=1$. If $i_{1}=1$, by Lemma 2.4, $v=e_{1} \boldsymbol{f}\left(x_{1}\right) x_{1}^{\alpha_{1}} e_{1} e_{\vec{i}^{\prime}, \vec{j}^{\prime}} w \bar{x}^{\beta}=0$, where 
$\vec{i}^{\prime}=\left(i_{2}, \ldots, i_{f}\right)$ and $\vec{j}^{\prime}=\left(j_{2}, \ldots, j_{f}\right)$. Now, we assume $i_{1} \neq 1$. Then

$$
\begin{aligned}
v & =e_{1} \boldsymbol{f}\left(x_{1}\right) x_{1}^{\alpha_{1}} e_{i_{1}, 1} e_{\vec{i}^{\prime}, \vec{j}^{\prime}} w \bar{x}^{\beta}=e_{1} e_{i_{1}, 1} \boldsymbol{f}\left(x_{1}\right) x_{1}^{\alpha_{1}} e_{\vec{i}^{\prime}, \vec{j}^{\prime}} w \bar{x}^{\beta} \\
& =e_{1}\left(1, i_{1}\right) \boldsymbol{f}\left(x_{1}\right) x_{1}^{\alpha_{1}} e_{\vec{i}^{\prime}, \vec{j}^{\prime}} w \bar{x}^{\beta}=e_{1} \boldsymbol{f}\left(x_{i}^{\prime}\right)(1, i) x_{1}^{\alpha_{1}} e_{{\overrightarrow{i^{\prime}}}^{\prime}, \vec{j}^{\prime}} w \bar{x}^{\beta}, \\
& =\boldsymbol{f}\left(x_{i}^{\prime}\right) e_{1}(1, i) x_{1}^{\alpha_{1}} e_{{\overrightarrow{i^{\prime}}}^{\prime}, \vec{j}^{\prime}} w \bar{x}^{\beta} \in J_{R} .
\end{aligned}
$$

This completes the proof of (2.17).

For $(\alpha, \beta) \in \mathbb{N}^{r} \times \mathbb{N}^{t}$, denote $\boldsymbol{f}\left(x^{\prime}\right)^{\alpha}=\boldsymbol{f}\left(x_{1}\right)^{\alpha_{1}} \cdots \boldsymbol{f}\left(x_{r}^{\prime}\right)^{\alpha_{r}}$ and $\boldsymbol{g}\left(\bar{x}^{\prime}\right)^{\beta}=\boldsymbol{g}\left(\bar{x}_{1}\right)^{\beta_{1}} \cdots \boldsymbol{g}\left(\bar{x}_{t}^{\prime}\right)^{\beta_{t}}$. Let $\mathbb{N}_{k}^{r}=\left\{\alpha \in \mathbb{N}^{r} \mid \alpha_{i} \leq k-1,1 \leq i \leq r\right\}$ and $\mathbb{N}_{k}^{t}=\left\{\alpha \in \mathbb{N}^{t} \mid \alpha_{i} \leq k-1,1 \leq i \leq r\right\}$.

Lemma 2.10. The affine walled Brauer algebra $\mathscr{B}_{r, t}^{\text {aff }}$ is a free $R$-module with $\mathcal{N}$ as its $R$-basis, where

$$
\begin{gathered}
\mathcal{N}=\bigcup_{f=0}^{\min \{m, n\}}\left\{\boldsymbol{f}\left(x^{\prime}\right)^{\alpha} x^{\gamma} c^{-1} e^{f} w d \bar{x}^{\delta} \boldsymbol{g}\left(\bar{x}^{\prime}\right)^{\beta} \mid(\alpha, \beta) \in \mathbb{N}^{r} \times \mathbb{N}^{t},(\gamma, \delta) \in \mathbb{N}_{k}^{r} \times \mathbb{N}_{k}^{t},\right. \\
\left.c, d \in \mathscr{D}_{r, t}^{f}, w \in \mathfrak{S}_{r-f} \times \overline{\mathfrak{S}}_{t-f}\right\} .
\end{gathered}
$$

Proof. The result follows from Theorem 2.6 since the transition matrix between $\mathcal{N}$ and $\mathcal{M}$ in (2.12) is invertible.

Lemma 2.11. Let $I$ be the two-sided ideal of $\mathscr{B}_{r, t}^{\text {aff }}$ generated by $\boldsymbol{f}\left(x_{1}\right)$ and $\boldsymbol{g}\left(\bar{x}_{1}\right)$ satisfying (2.5) -(2.7). If $\mathscr{B}_{r, t}^{\text {aff }}$ is admissible, then $S$ is an R-basis of $I$, where

$$
S=\left\{\boldsymbol{f}\left(x^{\prime}\right)^{\alpha} x^{\gamma} c^{-1} e^{f} w d \bar{x}^{\delta} \boldsymbol{g}\left(\bar{x}^{\prime}\right)^{\beta} \in \mathcal{N} \mid \alpha_{i}+\beta_{j} \neq 0 \text { for some } i, j\right\} .
$$

Proof. Let $M=\operatorname{span}_{R} S$. By Lemma 2.10, $\boldsymbol{f}\left(x_{1}\right) \mathscr{B}_{r, t}^{\text {aff }} \subseteq M$. For any positive integer $l$ with $1 \leq l<i$, by Lemma $2.8(2)$,

$$
\boldsymbol{f}\left(x_{i}^{\prime}\right) \boldsymbol{f}\left(x_{l}^{\prime}\right) \in \sum_{j=1}^{i-1} \boldsymbol{f}\left(x_{j}^{\prime}\right) \mathscr{B}_{r, t}^{\mathrm{aff}}+\boldsymbol{f}\left(x_{i}^{\prime}\right) D,
$$

such that $D \in \mathscr{B}_{r, t}^{\text {aff }}$ and the degree of $D$ is strictly less then $k$. Thus, $\boldsymbol{f}\left(x_{i}^{\prime}\right) \mathscr{B}_{r, t}^{\text {aff }} \subseteq M$ which follows from inductive assumption on $j$ with $1 \leq j \leq i-1$ and inductive assumption on degrees. This proves $J_{R} \subseteq M$. One can check $J_{L} \subseteq M$ similarly. By Proposition 2.9](3), $I=M$.

By abuse of notions, a regular monomial $\mathbf{m}$ in Definition 2.5 is also called a regular monomial of $\mathscr{B}_{k, r, t}$ if $0 \leq \alpha_{i}, \beta_{j} \leq k-1$ for all $i, j$ with $1 \leq i \leq r$ and $1 \leq j \leq t$. Obviously, the number of all such regular monomials is $k^{r+t}(r+t)$ !.

Theorem 2.12. The cyclotomic walled Brauer algebra $\mathscr{B}_{k, r, t}$ is free over $R$ with rank $k^{r+t}(r+t)$ ! if and only if $\mathscr{B}_{k, r, t}$ is admissible.

Proof. Let $M$ be the $R$-submodule of $\mathscr{B}_{k, r, t}$ spanned by all regular monomials of $\mathscr{B}_{k, r, t}$. By induction on degrees, it is routine to check that $M$ is left $\mathscr{B}_{k, r, t}$-module (cf. [19, Proposition 4.12] for $\left.\mathscr{B}_{r, t}^{\text {aff }}\right)$. Since $1 \in M$, we have $M=\mathscr{B}_{k, r, t}$. If $\mathscr{B}_{k, r, t}$ is not admissible, by Lemma 2.2, $e_{1}$ is an $R$-torsion element. Since $e_{1} \in M$, either $\mathscr{B}_{k, r, t}$ is not free or the rank of $\mathscr{B}_{k, r, t}$ is strictly less than $k^{r+t}(r+t)$ !. If $\mathscr{B}_{k, r, t}$ is admissible, by Lemmas 2.10 2.11, the set of all regular monomials of $\mathscr{B}_{k, r, t}$ is $R$-linear independent. Thus, $\mathscr{B}_{k, r, t}$ is free over $R$ with rank $k^{r+t}(r+t)$ !. 


\section{A WEAKLY CELlUlaR BASIS OF $\mathscr{B}_{2, r, t}$}

The aim of this section is to construct a weakly cellular basis of $\mathscr{B}_{2, r, t}$ in the sense of [11]. This basis will be used to set up a relationship between $\mathfrak{g l}_{m \mid n}$-Kac-modules and right cell modules of $\mathscr{B}_{2, r, t}$ in section 6 .

Recall that a composition of $r$ is a sequence of non-negative integers $\tau=\left(\tau_{1}, \tau_{2}, \ldots\right)$ such that $|\tau|:=\sum_{i} \tau_{i}=r$. If $\tau_{i} \geq \tau_{i+1}$ for all possible $i$ 's, then $\tau$ is called a partition. Similarly, a $k$-partition of $r$, or simply a multipartition of $r$, is an ordered $k$-tuple $\lambda=\left(\lambda^{(1)}, \lambda^{(2)}, \cdots, \lambda^{(k)}\right)$ of partitions with $|\lambda|:=\sum_{i=1}^{k}\left|\lambda^{(i)}\right|=r$. Let $\Lambda_{k}^{+}(r)$ be the set of all $k$-partitions of $r$. Let $\unlhd$ be the dominant order defined on $\Lambda_{k}^{+}(n)$ in the sense that $\lambda \unlhd \mu$ if and only if

$$
\sum_{h=1}^{\ell-1}\left|\lambda^{(h)}\right|+\sum_{j=1}^{i} \lambda_{j}^{(\ell)} \leq \sum_{k=1}^{\ell-1}\left|\mu^{(h)}\right|+\sum_{j=1}^{i} \mu_{j}^{(\ell)} \text { for } \ell \leq k \text { and all possible } i,
$$

where $\left|\lambda^{(0)}\right|=0$. Then $\Lambda_{k}^{+}(r)$ is a poset with $\unlhd$ as a partial order on it. In this paper, we always assume $k \in\{1,2\}$.

For each $\lambda \in \Lambda_{1}^{+}(r)$, the Young diagram $[\lambda]$ is a collection of boxes arranged in left-justified rows with $\lambda_{i}$ boxes in the $i$-th row of $[\lambda]$. A $\lambda$-tableau $\mathfrak{s}$ is obtained by inserting elements $i, 1 \leq i \leq r$ into $[\lambda]$ without repetition. A $\lambda$-tableau $\mathfrak{s}$ is said to be standard if the entries in $\mathfrak{s}$ increase both from left to right in each row and from top to bottom in each column. Let $\mathcal{T}^{s}(\lambda)$ be the set of all standard $\lambda$-tableaux. Let $\mathfrak{t}^{\lambda} \in \mathcal{T}^{s}(\lambda)$ be obtained from $[\lambda]$ by adding $1,2, \cdots, r$ from left to right along the rows of $[\lambda]$. Let $\mathfrak{t}_{\lambda} \in \mathcal{T}^{s}(\lambda)$ be obtained from $[\lambda]$ by adding $1,2, \cdots, r$ from top to bottom along the columns of $[\lambda]$. For example, if $\lambda=(3,2)$, then

$$
\mathfrak{t}^{\lambda}=\begin{array}{|l|l|l}
\hline 1 & 2 & 3 \\
\hline 4 & 5 &
\end{array}, \quad \text { and } \quad \mathfrak{t}_{\lambda}=\begin{array}{|l|l|l|}
\hline 1 & 3 & 5 \\
\hline 2 & 4 & \\
\hline
\end{array}
$$

If $\lambda \in \Lambda_{2}^{+}(r)$, then the corresponding Young diagram $[\lambda]$ is $\left(\left[\lambda^{(1)}\right],\left[\lambda^{(2)}\right]\right)$. In this case, a $\lambda$ tableau $\mathfrak{s}=\left(\mathfrak{s}_{1}, \mathfrak{s}_{2}\right)$ is obtained by inserting elements $i, 1 \leq i \leq r$ into $[\lambda]$ without repetition. A $\lambda$-tableau $\mathfrak{s}$ is said to be standard if the entries in $\mathfrak{s}_{i}, 1 \leq i \leq 2$ increase both from left to right in each row and from top to bottom in each column. Let $\mathcal{T}^{s}(\lambda)$ be the set of all standard $\lambda$-tableaux. Let $\mathfrak{t}^{\lambda} \in \mathcal{T}^{s}(\lambda)$ be obtained from $[\lambda]$ by adding $1,2, \cdots, r$ from left to right along the rows of $\left[\lambda^{(1)}\right]$ and then $\left[\lambda^{(2)}\right]$. Let $\mathfrak{t}_{\lambda} \in \mathcal{T}^{s}(\lambda)$ be obtained from $[\lambda]$ by adding $1,2, \cdots, r$ from top to bottom along the columns of $\left[\lambda^{(2)}\right]$ and then $\left[\lambda^{(1)}\right]$. For example, if $\lambda=((3,2),(3,1)) \in \Lambda_{2}^{+}(9)$, then

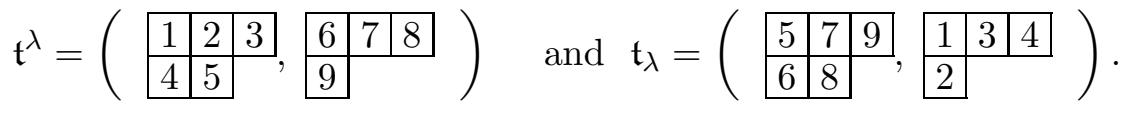

Recall that $\mathfrak{S}_{r}$ acts on the right of $1,2, \cdots, r$. Then $\mathfrak{S}_{r}$ acts on the right of a $\lambda$-tableau $\mathfrak{s}$ by permuting its entries. For example, if $\lambda=((3,2),(3,1)) \in \Lambda_{2}^{+}(9)$, and $w=s_{1} s_{2}$, then

$$
\mathfrak{t}^{\lambda} w=\left(\begin{array}{l|l|l|l|l|l|}
\hline 3 & 1 & 2 \\
\hline 4 & 5 & 6 & 7 & 8 \\
\hline 9 &
\end{array}\right) .
$$

Write $d(\mathfrak{s})=w$ for $w \in \mathfrak{S}_{r}$ if $\mathfrak{t}^{\lambda} w=\mathfrak{s}$. Then $d(\mathfrak{s})$ is uniquely determined by $\mathfrak{s}$. Let $w_{\lambda}=d\left(\mathfrak{t}_{\lambda}\right)$. The row stabilizer $\mathfrak{S}_{\lambda}$ of $\mathfrak{t}^{\lambda}$ for $\lambda \in \Lambda_{k}^{+}(r)$ is known as the Young subgroup of 
$\mathfrak{S}_{r}$ with respect to $\lambda$. It is the same as the Young subgroup $\mathfrak{S}_{\lambda_{\text {comp }}}$ with respect to the composition $\lambda_{\text {comp }}$, which is obtained from $\lambda$ by concatenation. For example, $\lambda_{\text {comp }}=(3,2,3,1)$ if $\lambda=((3,2),(3,1))$.

The level two degenerate Hecke $\mathscr{H}_{2, r}$ with defining parameters $u_{1}$ and $u_{2}$ is $\mathscr{H}_{r}^{\text {aff }} / I$, where $I$ is the two-sided ideal of $\mathscr{H}_{r}^{\text {aff }}$ generated by $\left(y_{1}-u_{1}\right)\left(y_{1}-u_{2}\right), u_{1}, u_{2} \in R$. By definition, $\mathscr{H}_{2, r}$ is an $R$-algebra generated by $s_{i}, 1 \leq i \leq r-1$ and $y_{j}, 1 \leq j \leq r$ such that

(1) $s_{i} s_{j}=s_{j} s_{i}, 1<|i-j|$,

(2) $y_{i} y_{\ell}=y_{\ell} y_{i}, 1 \leq i, \ell \leq r$,

(3) $s_{i} y_{i}-y_{i+1} s_{i}=-1, y_{i} s_{i}-s_{i} y_{i+1}=-1,1 \leq i \leq r-1$,

(4) $s_{j} s_{j+1} s_{j}=s_{j+1} s_{j} s_{j+1}, 1 \leq j \leq r-2$,

(5) $s_{i}^{2}=1,1 \leq i \leq r-1$,

(6) $\left(y_{1}-u_{1}\right)\left(y_{1}-u_{2}\right)=0$.

Following [3], we define $\pi_{\lambda}=\pi_{a}\left(u_{2}\right)$ and $\tilde{\pi}_{\lambda}=\pi_{a}\left(u_{1}\right)$ for $\lambda \in \Lambda_{2}^{+}(r)$ with $\left|\lambda^{(1)}\right|=a$, where for any $u \in R, \pi_{0}(u)=1$ and $\pi_{a}(u)=\prod_{i=1}^{a}\left(y_{i}-u\right)$ if $a>0$. Let

$$
w_{a}=\left(\begin{array}{cccccccc}
1 & 2 & \cdots & a & a+1 & a+2 & \cdots & r \\
r-a+1 & r-a+3 & \cdots & r & 1 & 2 & \cdots & r-a
\end{array}\right) .
$$

It is well-known that

$$
w_{a} s_{j}=s_{(j) w_{a}^{-1}} w_{a}, \quad \text { if } j \neq r-a .
$$

Let $\mathfrak{S}_{a, r-a}$ be the Young subgroup with respect to the composition $(a, r-a)$. Then

$$
R \mathfrak{S}_{a, r-a} w_{a}=w_{a} R \mathfrak{S}_{r-a, a} .
$$

For each composition $\lambda$ of $r$, we denote

$$
x_{\lambda}=\sum_{w \in \mathfrak{S}_{\lambda}} w, \quad y_{\lambda}=\sum_{w \in \mathfrak{S}_{\lambda}}(-1)^{\ell(w)} w,
$$

where $\ell(\cdot)$ is the length function on $\mathfrak{S}_{r}$. Assume $\lambda \in \Lambda_{2}^{+}(r)$ with $\left|\lambda^{(1)}\right|=a$. If we denote $\mu^{(i)}=\left(\lambda^{(i)}\right)^{\prime}$, the conjugate of $\lambda^{(i)}$ for $i=1,2$, then

$$
w_{a} x_{\mu^{(2)}} y_{\mu^{(1)}}=y_{\mu^{(1)}} x_{\mu^{(2)}} w_{a} .
$$

Remark 3.1. When we write $x_{\mu^{(2)}} y_{\mu^{(1)}}$, then $x_{\mu^{(2)}}$ (resp., $\left.y_{\mu^{(1)}}\right)$ is defined via symmetric group on $r-a$ letters $\{1,2, \cdots, r-a\}$ (resp., on $a$ letters $\{r-a+1, \cdots, r\})$. Similarly, when we write $y_{\mu^{(1)}} x_{\mu^{(2)}}$, then $y_{\mu^{(1)}}$ (resp., $\left.x_{\mu^{(2)}}\right)$ is defined via symmetric group on $a$ letters $\{1,2, \cdots, a\}$ (resp., on $r-a$ letters $\{a+1, a+2, \cdots, r\}$ ).

Definition 3.2. For any $\mathfrak{s}, \mathfrak{t} \in \mathcal{T}^{s}(\lambda)$ with $\lambda \in \Lambda_{2}^{+}(r)$, define

(1) $\mathfrak{x}_{\mathfrak{s t}}=d(\mathfrak{s})^{-1} \mathfrak{x}_{\lambda} d(\mathfrak{t})$, where $\mathfrak{x}_{\lambda}=\pi_{\lambda} x_{\lambda^{(1)}} y_{\lambda^{(2)}}$,

(2) $\mathfrak{y}_{\mathfrak{s t}}=d(\mathfrak{s})^{-1} \mathfrak{y}_{\lambda} d(\mathfrak{t})$, where $\mathfrak{y}_{\lambda}=\tilde{\pi}_{\lambda} x_{\lambda^{(1)}} y_{\lambda^{(2)}}$,

(3) $\overline{\mathfrak{x}}_{\mathfrak{s t}}=d(\mathfrak{s})^{-1} \overline{\mathfrak{x}}_{\lambda} d(\mathfrak{t})$, where $\overline{\mathfrak{x}}_{\lambda}=\pi_{\lambda} y_{\lambda^{(1)}} x_{\lambda^{(2)}}$,

(4) $\overline{\mathfrak{y}}_{\mathfrak{s t}}=d(\mathfrak{s})^{-1} \overline{\mathfrak{y}}_{\lambda} d(\mathfrak{t})$, where $\overline{\mathfrak{y}}_{\lambda}=\tilde{\pi}_{\lambda} y_{\lambda^{(1)}} x_{\lambda^{(2)}}$.

It is proven in [3] that $\mathscr{H}_{2, r}$ is a cellular algebra over $R$ in the sense of [12]. In this paper, we need the following cellular basis of $\mathscr{H}_{2, r}$ so as to construct a new weakly cellular basis of $\mathscr{B}_{2, r, t}$. 
Lemma 3.3. The set $S_{i}, i \in\{1,2,3,4\}$, are cellular bases of $\mathscr{H}_{2, r}$ in the sense of [12], where

(1) $S_{1}=\left\{\mathfrak{x}_{\mathfrak{s t}} \mid \lambda \in \Lambda_{2}^{+}(r), \mathfrak{s}, \mathfrak{t} \in \mathcal{T}^{s}(\lambda)\right\}$

(2) $S_{2}=\left\{\mathfrak{y}_{\mathfrak{s t}} \mid \lambda \in \Lambda_{2}^{+}(r), \mathfrak{s}, \mathfrak{t} \in \mathcal{T}^{s}(\lambda)\right\}$,

(3) $S_{3}=\left\{\overline{\mathfrak{x}}_{\mathfrak{s t}} \mid \lambda \in \Lambda_{2}^{+}(r), \mathfrak{s}, \mathfrak{t} \in \mathcal{T}^{s}(\lambda)\right\}$,

(4) $S_{4}=\left\{\overline{\mathfrak{y}}_{\mathfrak{s t}} \mid \lambda \in \Lambda_{2}^{+}(r), \mathfrak{s}, \mathfrak{t} \in \mathcal{T}^{s}(\lambda)\right\}$.

Proof. Let $S=\left\{x_{\mathfrak{s t}} \mid \mathfrak{s}, \mathfrak{t} \in \mathcal{T}^{s}(\lambda), \lambda \in \Lambda_{2}^{+}(r)\right\}$ and $x_{\mathfrak{s t}}=d(\mathfrak{s})^{-1} \pi_{\lambda} x_{\lambda^{(1)}} x_{\lambda^{(2)}} d(\mathfrak{t})$. It is proven in [3] that $S$ is a cellular basis of $\mathscr{H}_{2, r}$. If we use $y_{\lambda^{(2)}}$ instead of $x_{\lambda^{(2)}}$ in $x_{\mathfrak{s t}}$, we will get $\mathfrak{x}_{\mathfrak{s t}}$. However, for any $\mathfrak{s}=\left(\mathfrak{s}_{1}, \mathfrak{s}_{2}\right) \in \mathcal{T}^{s}(\lambda), d(\mathfrak{s})$ can be written uniquely as $d\left(\mathfrak{s}_{1}\right) d\left(\mathfrak{s}_{2}\right) d$ such that $d$ is a distinguished right coset representative of $\mathfrak{S}_{a} \times \mathfrak{S}_{r-a}$ in $\mathfrak{S}_{r}$ and $\mathfrak{s}_{i} \in \mathcal{T}^{s}\left(\lambda^{(i)}\right)$, where $a=\left|\lambda^{(1)}\right|$. So, the transition matrix between $S_{1}$ and $S$ is determined by the transition matrix between the cellular basis $\left\{d\left(\mathfrak{s}_{2}\right)^{-1} x_{\lambda^{(2)}} d\left(\mathfrak{t}_{2}\right) \mid \lambda^{(2)} \in \Lambda^{+}(r-a), \mathfrak{s}_{2}, \mathfrak{t}_{2} \in \mathcal{T}^{s}\left(\lambda^{(2)}\right)\right\}$ and $\left\{d\left(\mathfrak{s}_{2}\right)^{-1} y_{\lambda^{(2)}} d\left(\mathfrak{t}_{2}\right) \mid \lambda^{(2)} \in \Lambda^{+}(r-a), \mathfrak{s}_{2}, \mathfrak{t}_{2} \in \mathcal{T}^{s}\left(\lambda^{(2)}\right)\right\}$ of $R \mathfrak{S}_{r-a}$. Thus, $S_{1}$ is a basis of $\mathscr{H}_{2, r}$. One can check that $S_{1}$ is a cellular basis of $\mathscr{H}_{2, r}$ in the sense of [12] by mimicking Dipper-James-Murphy's arguments in the proof of Murphy basis for Hecke algebras of type $B$ in [8]. We leave the details to the readers. Finally, (2)-(4) can be verified similarly.

By Graham-Lehrer's results on the representation theory of cellular algebras in [12], one can define right cell modules of $\mathscr{H}_{2, r}$ via the cellular bases $S_{i}, i \in\{1,2,3,4\}$ in Lemma 3.3 . The corresponding right cell modules of $\mathscr{H}_{2, r}$ with respect to $S_{2}$ and $S_{4}$ are denoted by $\tilde{\Delta}(\lambda)$, and $\bar{\Delta}(\lambda)$.

For the simplification of discussion, we assume $\mathscr{H}_{2, r}$ is defined over $\mathbb{C}$ in Lemma 3.4.

Lemma 3.4. Suppose $a, b \in \mathbb{N}$. Then

(1) $\pi_{a}\left(u_{2}\right) \mathscr{H}_{2, r} \pi_{b}\left(u_{1}\right)=0$ whenever $a+b>r$ and $a, b \in \mathbb{Z}^{>0}$.

(2) $\pi_{a}\left(u_{2}\right) \mathscr{H}_{2, r} \pi_{r-a}\left(u_{1}\right)=\pi_{a}\left(u_{2}\right) w_{a} \pi_{r-a}\left(u_{1}\right) \mathbb{C S}_{r-a, a}$, where $\mathfrak{S}_{r-a, a}$ is as in (3.7).

(3) $\mathfrak{x}_{\lambda} \mathscr{H}_{2, r} \mathfrak{y}_{\mu^{\prime}}=0$ if $\lambda, \mu \in \Lambda_{2}^{+}(r)$ with $\lambda \triangleright \mu$,

(4) $\mathfrak{x}_{\lambda} \mathscr{H}_{2, r} \mathfrak{y}_{\lambda^{\prime}}=\operatorname{Span}_{\mathbb{C}}\left\{\mathfrak{x}_{\lambda} w_{\lambda} \mathfrak{y}_{\lambda^{\prime}}\right\}$ if $\lambda \in \Lambda_{2}^{+}(r)$.

(5) $\tilde{\Delta}\left(\lambda^{\prime}\right) \cong \mathfrak{x}_{\lambda} w_{\lambda} \mathfrak{y}_{\lambda^{\prime}} \mathscr{H}_{2, r}$.

Proof. (1)-(4) can be proven by arguments similar to those for Hecke algebras of type $B$ in [7. We only give details for (3) and (5).

If $\lambda \triangleright \mu$, then $\left|\lambda^{(1)}\right| \geq\left|\mu^{(1)}\right|$. If $\left|\lambda^{(1)}\right|>\left|\mu^{(1)}\right|$, then $\left|\mu^{(1)}\right| \neq r$ and the result follows from (1). When $\left|\lambda^{(1)}\right|=\left|\mu^{(1)}\right|$, by (2) together with corresponding result for the group algebras of symmetric groups, we have $\lambda^{(i)} \unlhd \mu^{(i)}$ for $i=1,2$ if $\mathfrak{x}_{\lambda} \mathscr{H}_{2, r} \mathfrak{y}_{\mu^{\prime}} \neq 0$. This proves (3).

There is a surjective $\mathscr{H}_{2, r}$-homomorphism from $\phi: \mathfrak{y}_{\lambda^{\prime}} \mathscr{H}_{2, r} \rightarrow \mathfrak{x}_{\lambda} w_{\lambda} \mathfrak{y}_{\lambda^{\prime}} \mathscr{H}_{2, r}$. Let $\mathscr{H}_{2, r}^{\triangleright \lambda^{\prime}}$ be the $\mathbb{C}$-submodule spanned by $\left\{\mathfrak{y}_{\mathfrak{s t}} \mid \mathfrak{s}, \mathfrak{t} \in \mathcal{T}^{s}(\mu), \mu \triangleright \lambda^{\prime}\right\}$. It follows from standard results on cellular algebras that $\mathscr{H}_{2, r}^{\triangleright \lambda^{\prime}}$ is a two-sided ideal of $\mathscr{H}_{2, r}$. So, $\mathfrak{y}_{\lambda^{\prime}} \mathscr{H}_{2, r}+\mathscr{H}_{2, r}^{\triangleright \lambda^{\prime}} / \mathscr{H}_{2, r}^{\triangleright \lambda^{\prime}}$ is isomorphic to a submodule of $\tilde{\Delta}\left(\lambda^{\prime}\right)$. If $\mathfrak{y}_{\mathfrak{s t}} \in \mathscr{H}_{2, r}^{\triangleright \lambda^{\prime}}$, we have $\mu \triangleright \lambda^{\prime}$ which is equivalent to $\lambda \triangleright \mu^{\prime}$. By (3), $x_{\lambda} w_{\lambda} \mathfrak{y}_{\mathfrak{s t}}=0$ and $\mathscr{H}_{2, r}^{\triangleright \lambda^{\prime}} \subset$ ker $\phi$. So, there is an epimorphism from $\mathfrak{y}_{\lambda^{\prime}} \mathscr{H}_{2, r}+\mathscr{H}_{2, r}^{\triangleright \lambda^{\prime}} / \mathscr{H}_{2, r}^{\triangleright \lambda^{\prime}}$ to $\mathfrak{x}_{\lambda} w_{\lambda} \mathfrak{y}_{\lambda^{\prime}} \mathscr{H}_{2, r}$. Mimicking arguments on classical Specht modules for Hecke algebra of type $B$ in [7, we know that $\mathfrak{x}_{\lambda} w_{\lambda} \mathfrak{y}_{\lambda^{\prime}} \mathscr{H}_{2, r}$ has a basis $\left\{\mathfrak{x}_{\lambda} w_{\lambda} \mathfrak{y}_{\lambda^{\prime}} d(\mathfrak{t}) \mid \mathfrak{t} \in \mathcal{T}^{s}\left(\lambda^{\prime}\right)\right\}$. So,

$$
\operatorname{dim}_{\mathbb{C}} \tilde{\Delta}\left(\lambda^{\prime}\right)=\operatorname{dim}_{\mathbb{C}} \mathfrak{x}_{\lambda} w_{\lambda} \mathfrak{y}_{\lambda^{\prime}} \mathscr{H}_{2, r}=\# \mathcal{T}^{s}\left(\lambda^{\prime}\right),
$$


forcing $\mathfrak{y}_{\lambda^{\prime}} \mathscr{H}_{2, r}+\mathscr{H}_{2, r}^{\unrhd \lambda^{\prime}} / \mathscr{H}_{2, r}^{\unrhd \lambda^{\prime}} \cong \mathfrak{x}_{\lambda} w_{\lambda} \mathfrak{y}_{\lambda^{\prime}} \mathscr{H}_{2, r} \cong \tilde{\Delta}\left(\lambda^{\prime}\right)$.

Now, we use cellular bases $S_{i}$ of $\mathscr{H}_{2, r}$ in Lemma 3.3 to construct a weakly cellular basis of $\mathscr{B}_{2, r, t}$ over an arbitrary field in the sense of [11]. We remark that when we use results on level two degenerate Hecke algebra for $\mathscr{B}_{2, r, t}$, we should keep in mind that $x_{1}, \bar{x}_{1} \in \mathscr{B}_{2, r t}$ should be regarded as $-y_{1} \in \mathscr{H}_{2, r}$ and $\mathscr{H}_{2, t}$, respectively. Therefore, we have to use $-u_{i}$ and $-\bar{u}_{i}$ instead of $u_{i}$ and $\bar{u}_{i}$.

Fix $r, t, f \in \mathbb{Z}^{>0}$ with $f \leq \min \{r, t\}$. In contrast to (2.11), we define

$$
\mathcal{D}_{r, t}^{f}=\left\{s_{r-f+1, i_{r-f+1}} \bar{s}_{t-f+1, j_{t-f+1}} \cdots s_{r, i_{r}} \bar{s}_{t, j_{t}} \mid r \geq i_{r}>\cdots>i_{r-f+1}, j_{k} \geq k+f-t\right\} .
$$

For each $c \in \mathcal{D}_{r, t}^{f}$ as in (3.10), let $\kappa_{c}$ be the $r$-tuple

$$
\kappa_{c}=\left(k_{1}, \ldots, k_{r}\right) \in\{0,1\}^{r} \text { such that } k_{i}=0 \text { unless } i=i_{r}, i_{r-1}, \ldots, i_{r-f+1} .
$$

Note that $\kappa_{c}$ may have more than one choice for a fixed $c$, and it may be equal to $\kappa_{d}$ although $c \neq d$ for $c, d \in \mathcal{D}_{r, t}^{f}$. Let $\mathbf{N}_{f}=\left\{\kappa_{c} \mid c \in \mathcal{D}_{r, t}^{f}\right\}$. If $\kappa_{c} \in \mathbf{N}_{f}$, define $x^{\kappa_{c}}=\prod_{i=1}^{r} x_{i}^{k_{i}}$. In [19], we consider poset $\left(\Lambda_{2, r, t}, \unrhd\right)$, where

$$
\Lambda_{2, r, t}=\left\{(f, \lambda, \mu) \mid(\lambda, \mu) \in \Lambda_{2}^{+}(r-f) \times \Lambda_{2}^{+}(t-f), 0 \leq f \leq \min \{r, t\}\right\},
$$

such that $(f, \lambda, \mu) \unrhd(\ell, \alpha, \beta)$ for $(f, \lambda, \mu),(\ell, \alpha, \beta) \in \Lambda_{2, r, t}$ if either $f>\ell$ or $f=\ell$ and $\lambda \unrhd_{1} \alpha$, and $\mu \unrhd_{2} \beta$, and in case $f=\ell$, the orders $\unrhd_{1}$ and $\unrhd_{2}$ are dominant orders on $\Lambda_{2}^{+}(r-f)$ and $\Lambda_{2}^{+}(t-f)$ respectively. For each $(f, \mu, \nu) \in \Lambda_{2, r, t}$, let

$$
\delta(f, \mu, \nu)=\left\{\left(\mathfrak{t}, c, \kappa_{c}\right) \mid \mathfrak{t}=\left(\mathfrak{t}^{(1)}, \mathfrak{t}^{(2)}\right) \in \mathcal{T}^{s}(\mu) \times \mathcal{T}^{s}(\nu), c \in \mathcal{D}_{r, t}^{f} \text { and } \kappa_{c} \in \mathbf{N}_{f}\right\} .
$$

Definition 3.5. For any $\left(\mathfrak{s}, d, \kappa_{d}\right),\left(\mathfrak{t}, c, \kappa_{c}\right) \in \delta(f, \mu, \nu)$ with $(f, \mu, \nu) \in \Lambda_{2, r, t}$, define

$$
C_{\left(\mathfrak{s}, d, \kappa_{d}\right)\left(\mathfrak{t}, c, \kappa_{c}\right)}=x^{\kappa_{d}} d^{-1} \mathfrak{e}^{f} \mathfrak{n}_{\mathfrak{s t}} c x^{\kappa_{c}},
$$

where, in contrast to notation $e^{f}$ in (2.10), we define $\mathfrak{e}^{f}=e_{r, t} e_{r-1, t-1} \cdots e_{r-f+1, t-f+1}$ if $f \geq 1$ and $\mathfrak{e}^{0}=1$, and $\mathfrak{n}_{\mathfrak{s t}}=\mathfrak{y}_{\mathfrak{s}^{(1)} \mathfrak{t}^{(1)}} \overline{\mathfrak{y}}_{\mathfrak{s}^{(2)} \mathfrak{t}^{(2)}}$ if $\mathfrak{s}=\left(\mathfrak{s}^{(1)}, \mathfrak{s}^{(2)}\right)$ and $\mathfrak{t}=\left(\mathfrak{t}^{(1)}, \mathfrak{t}^{(2)}\right)$ are in $\mathcal{T}^{s}(\mu) \times \mathcal{T}^{s}(\nu)$.

Note that $\mathfrak{n}_{\mathfrak{s t}}$ in Definition 3.5 are defined via cellular basis elements of $\mathscr{H}_{2, r-f}$ and $\mathscr{H}_{2, t-f}$ in Lemma $3.3(2)$ (4). Since $x_{i}$ and $\bar{x}_{j}$ do not commute each other, a cellular basis element of $\mathscr{H}_{2, r-f}$ is always put on the left. Further, we need to use $x_{i},-u_{1},-u_{2}$ (resp. $\left.\bar{x}_{i},-\bar{u}_{1},-\bar{u}_{2}\right)$ instead of $-y_{i}, u_{1}, u_{2}$ in Lemma 3.3 .

Theorem 3.6. If $\mathscr{B}_{2, r, t}$ is admissible, then the set

$$
\mathscr{C}=\left\{C_{\left(\mathfrak{s}, \kappa_{c}, c\right)\left(\mathfrak{t}, \kappa_{d}, d\right)} \mid\left(\mathfrak{s}, \kappa_{c}, c\right),\left(\mathfrak{t}, \kappa_{d}, d\right) \in \delta(f, \lambda), \forall(f, \lambda) \in \Lambda_{2, r, t}\right\}
$$

is a weakly cellular basis $\mathscr{B}_{2, r, t}$ over $R$ in the sense of [11].

Proof. Let $S$ be the cellular basis of $\mathscr{H}_{2, r-f}\left(\right.$ resp. $\mathscr{H}_{2, t-f}$ ) for $0 \leq f \leq \min \{r, t\}$ defined in the proof of Lemma 3.3. If we use $S$ instead of the cellular basis $S_{2}$ of $\mathscr{H}_{2, r-f}$ and $S_{4}$ of $\mathscr{H}_{2, t-f}$ in Lemma 3.3, we will obtain the weakly cellular basis of $\mathscr{B}_{2, r, t}$ over $R$ in [19, Theorem 6.12] provided that $R=\mathbb{C}$ and $u_{1}=-p, u_{2}=m-q, \bar{u}_{1}=q$ and $\bar{u}_{2}=p-n$ with $r+t \leq \min \{m, n\}$. Since $\mathscr{B}_{2, r, t}$ is admissible, by Theorem 2.12 , the rank of $\mathscr{B}_{2, r, t}$ is $2^{r+t}(k+t)$ !. As pointed in [19, Remark 6.13], [19, Theorem 6.12] holds over $R$ with arbitrary 
parameters $u_{1}, u_{2}, \bar{u}_{1}, \bar{u}_{2}$ if the rank of $\mathscr{B}_{2, r, t}$ is $2^{r+t}(r+t)$ !. Thus, $\mathscr{C}$ is an $R$-basis of $\mathscr{B}_{2, r, t}$. Further, the weakly cellularity of $\mathscr{B}_{2, r, t}$ depends only on cellular bases of $\mathscr{H}_{2, r-f}$ and $\mathscr{H}_{2, t-f}$ and does not depend on the explicit descriptions of cellular bases of $\mathscr{H}_{2, r-f}$ and $\mathscr{H}_{2, t-f}$. (cf. the proof of [19, Theorem 6.12]). So, all arguments for the proof of [19, Theorem 6.12] can be used smoothly to prove that $\mathscr{C}$ is a weakly cellular basis $\mathscr{B}_{2, r, t}$ over $R$.

Suppose $\mathscr{B}_{2, r, t}$ is defined over a field $F$. By Theorem 3.6, one can define right cell modules $C(f, \mu, \nu)$ with respect to $(f, \mu, \nu) \in \Lambda_{2, r, t}$ for $\mathscr{B}_{2, r, t}$. Let $\phi_{f, \mu, \nu}$ be the corresponding invariant form on $C(f, \mu, \nu)$ and let $D^{f, \mu, \nu}=C(f, \mu, \nu) / \operatorname{Rad} \phi_{f, \mu, \nu}$, where $\operatorname{Rad} \phi_{f, \mu, \nu}$ is the radical of $\phi_{f, \mu, \nu}$. By Graham-Lehrer's results in [12] (a weakly cellular algebra has similar representation theory of a cellular algebra in [12]), $D^{f, \mu, \nu}$ is either 0 or irreducible and all non-zero $D^{f, \mu, \nu}$ consist of a complete set of pair-wise non-isomorphic irreducible $\mathscr{B}_{2, r, t}$-modules. Let $\tilde{\Delta}(\mu)$ (resp. $\bar{\Delta}(\nu)$ ) be the cell module of $\mathscr{H}_{2, r-f}\left(\right.$ resp. $\left.\mathscr{H}_{2, t-f}\right)$ defined via $S_{2}$ and $S_{4}$ in Lemma 3.3 . Similarly, one has the notations $D^{\mu}$ and $\bar{D}^{\nu}$, respectively.

Proposition 3.7. Suppose that $\mathscr{B}_{2, r, t}$ is admissible over $F$. For any $(f, \mu, \nu) \in \Lambda_{2, r, t}$, $D^{f, \mu, \nu} \neq 0$ if and only if

(1) $D^{\mu} \neq 0$ and $\bar{D}^{\nu} \neq 0$,

(2) $f \neq r$ provided $r=t$ and $\omega_{0}=\omega_{1}=0$.

Proof. The result can be proven by arguments similar to those for Lemmas 7.3-7.4 in [19]. $\square$

Remark 3.8. By arguments similar to those for Theorem 3.6, one can lift cellular bases of $\mathscr{H}_{k, r}$ and $\mathscr{H}_{k, t}$ in [3] to obtain a weakly cellular basis of $\mathscr{B}_{k, r, t}$ over $R$, provide that $\mathscr{B}_{k, r, t}$ is admissible. Further, it is not difficult to prove a result which is similar to Proposition 3.7 for $\mathscr{B}_{k, r, t}$ over an arbitrary field $F$ with characteristic char $F$ either zero or positive. Let $\mathbf{u}=\left(u_{1}, \cdots, u_{k}\right) \in F^{k}$ such that $u_{i}=d_{i} \cdot 1_{F}$ and $0 \leq d_{i}<\operatorname{char} F$ for $1 \leq i \leq k$. Kleshchev [15] has shown that the simple $\mathscr{H}_{k, n}(\mathbf{u})$-modules are labeled by a set of multipartitions which gives the same Kashiwara crystal as the set of $\mathbf{u}$-Kleshchev multipartitions of $n$ in [1, 2]. Thus, the simple $\mathscr{B}_{k, r, t}$-modules are labeled by the set $\{(f, \mu, \nu)\}$, where $(1) 0 \leq f \leq \min \{r, t\}$, (2) $\mu$ 's are Kleshchev multipartitions of $r-f$ with respect to $\mathbf{u}$, (3) $\nu$ 's are Kleshchev multipartitions of $t-f$ with respect to $\overline{\mathbf{u}}:=\left(\bar{u}_{1}, \bar{u}_{2}, \cdots, \bar{u}_{k}\right),(4) f \neq r$ if $r=t$ and $\omega_{i}=0$ for $0 \leq i \leq k-1$. It is pointed in [3] that one can modify the proof of [9, Theorem 1.1], or [1, Theorem 1.3], to show that when $\mathscr{B}_{k, r, t}$ is admissible, the simple $\mathscr{B}_{k, r, t}$-modules are always labeled by the $(f, \mu, \nu) \in \Lambda_{k, r, t}$ with $0 \leq f \leq \min \{r, t\}$ and $\mu$ (resp. $\nu$ ) are Kleshchev multipartitons with respect to $\mathbf{u}$ (resp. $\overline{\mathbf{u}}$ ) and $f \neq r$ if $r=t$ and $\omega_{i}=0$ for $1 \leq i \leq r$. However, we are not claiming that $D^{(f, \mu, \nu)} \neq 0$ for the multipartitions $\mu, \nu$ which Kleshchev [15] uses to label the simple $\mathscr{H}_{k, r-f}(\mathbf{u})$-modules (resp. $\mathscr{H}_{k, r-f}(\overline{\mathbf{u}})$-modules).

We recall the definition of Kleshchev bipartitions over $\mathbb{C}$ as follows (see e.g., [25]), which will be used in sections 5-6. Fix $u_{1}, u_{2} \in \mathbb{C}$ with $u_{1}-u_{2} \in \mathbb{N}$. Then $\lambda=\left(\lambda^{(1)}, \lambda^{(2)}\right) \in \Lambda_{2}^{+}(r)$ is called a Kleshchev bipartition [25] with respect to $u_{1}, u_{2}$ if

$$
\lambda_{u_{1}-u_{2}+i}^{(1)} \leq \lambda_{i}^{(2)} \text { for all possible } i .
$$


If $u_{1}-u_{2} \notin \mathbb{Z}$, all bipartitions of $r$ are Kleshchev bipartitions. A pair of bipartitions $(\mu, \nu)$ is Kleshchev if both $\mu$ and $\nu$ are Kleshchev bipartitions in the sense of (3.15) with respect to the parameters $u_{1}, u_{2}$ and $\bar{u}_{1}, \bar{u}_{2}$. The following result will be used in section 6 .

Proposition 3.9. Suppose $\mathscr{B}_{2, r, t}$ is admissible over $\mathbb{C}$. For each $(f, \mu, \nu) \in \Lambda_{2, r, t}$, let

$$
\tilde{C}(f, \mu, \nu):=\mathfrak{e}^{f} \mathfrak{x}_{\mu^{\prime}} \overline{\mathfrak{x}}_{\nu^{\prime}} w_{\mu^{\prime}} w_{\nu^{\prime}} \mathfrak{y}_{\mu} \overline{\mathfrak{y}}_{\nu} \mathscr{B}_{2, r, t} \quad\left(\bmod \mathscr{B}_{2, r, t}^{f+1}\right),
$$

where $\mathscr{B}_{2, r, t}^{f+1}$ is the two-sided ideal of $\mathscr{B}_{2, r, t}$ generated by $\mathfrak{e}^{f+1}$. Then $C(f, \mu, \nu) \cong \tilde{C}(f, \mu, \nu)$.

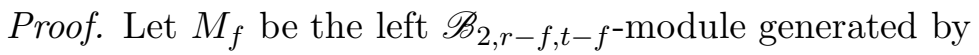

$$
V_{r, t}^{f}=\left\{\mathfrak{e}^{f} d x^{\kappa_{d}} \mid\left(d, \kappa_{d}\right) \in \mathcal{D}_{r, t}^{f} \times \mathbf{N}_{f}\right\} .
$$

By [19, Proposition 6.10], $M_{f}=\mathfrak{e}^{f} \mathscr{B}_{2, r, t}$. By [19, Lemma 6.9], one can use $\mathscr{H}_{2, r-f} \otimes \mathscr{H}_{2, t-f}$ instead of $\mathscr{B}_{2, r-f, t-f}$ in $\mathfrak{x}_{\mu^{\prime}} \overline{\mathfrak{x}}_{\nu^{\prime}} w_{\mu^{\prime}} w_{\nu^{\prime}} \mathfrak{y}_{\mu} \overline{\mathfrak{y}}_{\nu} M_{f}\left(\bmod \mathscr{B}_{2, r, t}^{f+1}\right)$. Now, the required isomorphism follows from Lemma $3.4(5)$.

\section{Super Schur-Weyl DuAlity}

The aim of this section is to generalize super Schur-Weyl duality between general linear Lie superalgebra $\mathfrak{g l}_{m \mid n}$ and $\mathscr{B}_{2, r, t}$ to the case $r+t>\min \{m, n\}$. Throughout, let $I_{0}=\{1, \ldots, m\}$, $I_{1}=\{m+1, \ldots, m+n\}$ and $I=I_{0} \cup I_{1}$.

For any pairs $(i, j) \in I \times I$, let $E_{i j}$ be the matrix unit with parity $\left[E_{i j}\right]=[i]+[j]$, where $[i]=a$ if $i \in I_{a}, a=0,1$. The general linear Lie superalgebra $\mathfrak{g l}_{m \mid n}$ over $\mathbb{C}$, denoted by $\mathfrak{g}$, is $\mathfrak{g}_{-1} \oplus \mathfrak{g}_{0} \oplus \mathfrak{g}_{1}$, where

$$
\begin{aligned}
& \mathfrak{g}_{-1}=\operatorname{span}_{\mathbb{C}}\left\{E_{i, j} \mid i \in I_{1}, j \in I_{0}\right\}, \mathfrak{g}_{1}=\operatorname{span}_{\mathbb{C}}\left\{E_{i, j} \mid i \in I_{0}, j \in I_{1}\right\}, \\
& \mathfrak{g}_{0}=\operatorname{span}_{\mathbb{C}}\left\{E_{i, j} \mid i, j \in I_{0} \text { or } i, j \in I_{1}\right\} .
\end{aligned}
$$

The Cartan subalgebra $\mathfrak{h}$ of $\mathfrak{g}$ is the $\mathbb{C}$-space with basis $\left\{E_{i i} \mid i \in I\right\}$. Let $\mathfrak{h}^{*}$ be the dual space of $\mathfrak{h}$ with dual basis $\left\{\varepsilon_{i} \mid i \in I\right\}$. Then any $\xi \in \mathfrak{h}^{*}$, called a weight of $\mathfrak{g}$, can be written as

$$
\xi=\sum_{i \in I_{0}} \xi_{i}^{L} \varepsilon_{i}+\sum_{i \in I_{1}} \xi_{i-m}^{R} \varepsilon_{i} \text { with } \xi_{i}^{L}, \xi_{j}^{R} \in \mathbb{C} .
$$

Denote $\xi$ by $\left(\xi_{1}^{L}, \ldots, \xi_{m}^{L} \mid \xi_{1}^{R}, \ldots, \xi_{n}^{R}\right)$. If both $\xi_{i}^{L}-\xi_{i+1}^{L} \in \mathbb{N}$ and $\xi_{j}^{R}-\xi_{j+1}^{R} \in \mathbb{N}$ for all possible $i, j$, then $\xi$ is called integral dominant. Let $P^{+}$be the set of integral dominant weights. For any $\xi \in P^{+}$, let

$$
\xi^{\rho}:=\xi+\rho=\left(\xi_{1}^{L, \rho}, \ldots, \xi_{m}^{L, \rho} \mid \xi_{1}^{R, \rho}, \ldots, \xi_{n}^{R, \rho}\right),
$$

where $\rho=(0,-1, \ldots, 1-m \mid m-1, m-2, \ldots, m-n)$. Following [14, let

$$
\ell=\#\left\{(i, j) \mid \xi_{i}^{L, \rho}+\xi_{j}^{R, \rho}=0,1 \leq i \leq m, 1 \leq j \leq n\right\} .
$$

Then $\xi$ is called an $\ell$-fold atypical weight if $\ell>0$. Otherwise, $\xi$ is called a typical weight.

Example 4.1. For any $p, q \in \mathbb{C}$, let $\lambda_{p q}=(p, \ldots, p \mid-q, \ldots,-q)$. Then $\lambda_{p q}$ is a typical weight if and only if

$$
p-q \notin \mathbb{Z} \text { or } p-q \leq-m \text { or } p-q \geq n \text {. }
$$


The current $q$ should be regarded as $q+m$ in [5, IV]. In the remaining part of this paper, $\lambda_{p q}$ is always a typical weight in the sense of (4.4).

Let $V=\mathbb{C}^{m \mid n}$ be the natural $\mathfrak{g}$-module with natural basis $\left\{v_{i} \mid i \in I\right\}$ such that $v_{i}$ has parity $\left[v_{i}\right]=[i]$. Then the dual space $V^{*}$, which has the dual basis $\left\{\bar{v}_{i} \mid i \in I\right\}$, is a left $\mathfrak{g}$-module such that

$$
E_{a b} \bar{v}_{i}=-(-1)^{[a]([a]+[b])} \delta_{i a} \bar{v}_{b} \text { for any }(a, b) \in I \times I .
$$

In particular, the weight of $\bar{v}_{i}$ is $-\epsilon_{i}$. For the simplicity of notation, we set $W=V^{*}$.

Definition 4.2. Fix $r, t \in \mathbb{Z}^{>0}$. Let $V^{r t}=V^{\otimes r} \otimes W^{\otimes t}$ and $M_{p q}^{r t}=V^{\otimes r} \otimes K_{\lambda_{p q}} \otimes W^{\otimes t}$, where $K_{\lambda_{p q}}$ is the Kac-module [14] with respect to the highest weight $\lambda_{p q}$ in Example 4.1.

Let $\pi: M_{p q}^{r t} \rightarrow V^{r t}$ be the projection such that, for any $v \in M_{p q}^{r t}, \pi(v)$ is the vector obtained from $v$ by deleting the tensor factor in $K_{\lambda_{p q}}$. Let $v_{p q}$ be the highest weight vector of $K_{\lambda_{p q}}$ with highest weight $\lambda_{p q}$. Then $v_{p q}$ is unique up to a scalar. It is well-known (e.g. see [5]) that $K_{\lambda_{p q}}$ is $2^{m n}$-dimensional with a basis

$$
B=\left\{b^{\sigma}:=\prod_{i=1}^{n} \prod_{j=1}^{m} E_{m+i, j}^{\sigma_{i j}} v_{p q} \mid \sigma=\left(\sigma_{i j}\right)_{i, j=1}^{n, m} \in\{0,1\}^{n \times m}\right\},
$$

where the products are taken in any fixed order. Define

$$
\begin{aligned}
I(m \mid n, r) & =\left\{\mathbf{i} \mid \mathbf{i}=\left(i_{r}, i_{r-1}, \cdots, i_{1}\right), i_{j} \in I, 1 \leq j \leq r\right\}, \\
\bar{I}(m \mid n, t) & =\left\{\mathbf{j} \mid \mathbf{j}=\left(j_{1}, j_{2}, \cdots, j_{t}\right), j_{i} \in I, 1 \leq i \leq t\right\} .
\end{aligned}
$$

If $(\mathbf{i}, b, \mathbf{j}) \in I(m \mid n, r) \times B \times \bar{I}(m \mid n, t)$, we define

$$
v_{\mathbf{i}, b, \mathbf{j}}=v_{i_{r}} \otimes v_{i_{r-1}} \otimes \cdots \otimes v_{i_{1}} \otimes b \otimes \bar{v}_{j_{1}} \otimes \bar{v}_{j_{2}} \otimes \cdots \otimes \bar{v}_{j_{t}} \in M_{p q}^{r t}
$$

Lemma 4.3. Let $B_{M}=\left\{v_{\mathbf{i}} \otimes b \otimes \bar{v}_{\mathbf{j}} \mid(\mathbf{i}, b, \mathbf{j}) \in I(m \mid n, r) \times B \times \bar{I}(m \mid n, t)\right\}$. Then $B_{M}$ is $a$ basis of $M_{p q}^{r t}$.

Denote by $U(\mathfrak{g})$ the universal enveloping algebra of $\mathfrak{g}$. Then $M_{p q}^{r t}$ is a left $U(\mathfrak{g})$-module. Let $J=J_{1} \cup\{0\} \cup J_{2}$ with $J_{1}=\{r, \ldots, 2,1\}$ and $J_{2}=\{\overline{1}, \overline{2}, \ldots, \bar{t}\}$. Then $(J, \prec)$ is a total ordered set with

$$
r \prec r-1 \prec \cdots \prec 1 \prec 0 \prec \overline{1} \prec \cdots \prec \bar{t} .
$$

For any $a, b \in J$ with $a \prec b$, define $\pi_{a b}: U(\mathfrak{g})^{\otimes 2} \rightarrow U(\mathfrak{g})^{\otimes(r+t+1)}$ by

$$
\pi_{a b}(x \otimes y)=1 \otimes \cdots \otimes 1 \otimes \stackrel{a-\text { th }}{x} \otimes 1 \otimes \cdots \otimes 1 \otimes \stackrel{b-\text { th }}{y} \otimes 1 \otimes \cdots \otimes 1 .
$$

Let $\Omega$ be a Casimir element in $\mathfrak{g}^{\otimes 2}$ given by

$$
\Omega=\sum_{i, j \in I}(-1)^{[j]} E_{i j} \otimes E_{j i} .
$$

In [19], we define operators $s_{i}, \bar{s}_{j}, x_{1}, \bar{x}_{1}$ and $e_{1}$ acting on the right of $M_{p q}^{r t}$ via the following formulae:

$$
\begin{aligned}
& s_{i}=\left.\pi_{i+1, i}(\Omega)\right|_{M_{p q}^{r t}}(1 \leq i<r), \quad \bar{s}_{j}=\left.\pi_{\bar{j}, \overline{j+1}}(\Omega)\right|_{M_{p q}^{r t}}(1 \leq j<t), \\
& x_{1}=-\left.\pi_{10}(\Omega)\right|_{M_{p q}^{r t}}, \quad \bar{x}_{1}=-\left.\pi_{0 \overline{1}}(\Omega)\right|_{M_{p q}^{r t}}, \quad e_{1}=-\left.\pi_{1 \overline{1}}(\Omega)\right|_{M_{p q}^{r t}} .
\end{aligned}
$$


Then there is an algebra homomorphism $\phi: \mathscr{B}_{2, r, t} \rightarrow \operatorname{End}_{U(\mathfrak{g})}\left(M_{p q}^{r t}\right)$ sending the generators $s_{i}, \bar{s}_{j}, x_{1}, \bar{x}_{1}$ and $e_{1}$ to the operators $s_{i}, \bar{s}_{j}, x_{1}, \bar{x}_{1}$ and $e_{1}$ as above [19]. In this case, we need to use $-p, m-q$, and $q, p-n$ instead of $u_{1}, u_{2}, \bar{u}_{1}$ and $\bar{u}_{2}$ respectively in Definition 2.1 for $k=2$. Further, $\omega_{0}=m-n, \omega_{1}=n q-m p$ and $\omega_{a}=(m-p-q) \omega_{a-1}-p(q-m) \omega_{a-2}$ for $a \geq 2$ and $\bar{\omega}_{a}$ 's are determined by [19, Corollary 4.3]. Thus, $\mathscr{B}_{2, r, t}$ is admissible in the sense of Definition 2.3. By Theorem 2.12, $\operatorname{dim}_{\mathbb{C}} \mathscr{B}_{2, r, t}=2^{r+t}(r+t)$ !. We will always consider $\mathscr{B}_{2, r, t}$ as above in the remaining part of this paper.

Theorem 4.4. [19, Theorem 5.16] Fix $r, t \in \mathbb{Z}^{>0}$ with $r+t \leq \min \{m, n\}$. Then $\operatorname{End}_{\mathfrak{g}}\left(M_{p q}^{r t}\right) \cong \mathscr{B}_{2, r, t}$.

Theorem 4.5. [5, IV, Theorem 3.13] If $0<r \leq \min \{m, n\}$, then $\operatorname{End}_{U(\mathfrak{g})}\left(M_{p q}^{r 0}\right) \cong \mathscr{H}_{2, r}$, the level two Hecke algebra with defining parameters $u_{1}=-p$ and $u_{2}=m-q$.

Theorem 4.6. (Super Schur-Weyl duality) Keep the condition (4.4). The algebra homomorphism $\phi_{1}: \mathscr{B}_{2, r, t} \rightarrow \operatorname{End}_{\mathfrak{g}}\left(M_{p q}^{r t}\right)$ is surjective. It it injective if and only $r+t \leq \min \{m, n\}$.

Proof. By Theorem 4.4, it suffices to prove that $\phi_{1}$ is surjective and is not injective if $r+t>\min \{m, n\}$. As in [6, (7.16)], the map flip $p_{r, t}$ defined by the following commutative diagram is a $\mathfrak{g}$-module isomorphism

$$
\begin{aligned}
& \operatorname{End}_{\mathbb{C}}\left(V^{\otimes r} \otimes K_{\lambda_{p q}} \otimes\left(V^{*}\right)^{\otimes t}\right) \quad \stackrel{\text { flip }_{r, t}}{\longrightarrow} \quad \operatorname{End}_{\mathbb{C}}\left(V_{\|}^{\otimes r} \otimes K_{\lambda_{p q}} \otimes V^{\otimes t}\right) \\
& \operatorname{End}_{\mathbb{C}}\left(V^{\otimes r} \otimes K_{\lambda_{p q}}\right) \otimes \operatorname{End}_{\mathbb{C}}\left(\left(V^{*}\right)^{\otimes t}\right) \stackrel{f \otimes g^{*} \mapsto f \otimes g}{\longrightarrow} \operatorname{End}_{\mathbb{C}}\left(V^{\otimes r} \otimes K_{\lambda_{p q}}\right) \otimes \operatorname{End}_{\mathbb{C}}\left(V^{\otimes t}\right) .
\end{aligned}
$$

Note that $\mathscr{H}_{2, r+t}\left(\right.$ denoted as $H_{r+t}^{p, q}$ in [5, IV]) is a subspace of $\operatorname{End}_{\mathbb{C}}\left(V^{\otimes r} \otimes K_{\lambda_{p q}} \otimes V^{\otimes t}\right)$, thus (4.12) induces the following commutative diagram

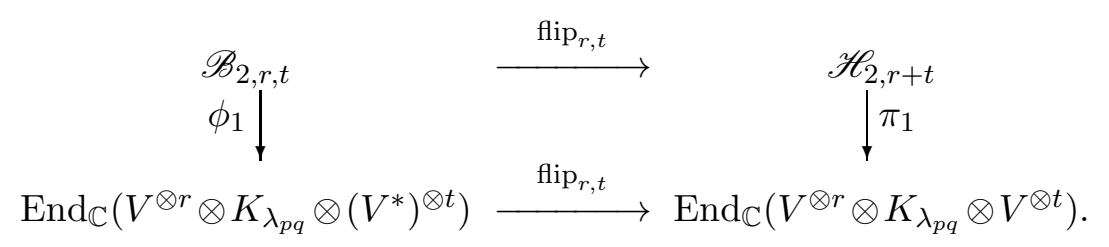

By Theorem 2.12 for $k=2, \operatorname{dim}_{\mathbb{C}} \mathscr{B}_{2, r, t}=2^{r+t}(r+t)$ !. This implies that the top map is a bijection, and the bottom map is a $\mathfrak{g}$-module isomorphism, which induces an isomorphism between two subspaces $\operatorname{End}_{\mathfrak{g}}\left(V^{\otimes r} \otimes K_{\lambda_{p q}} \otimes\left(V^{*}\right)^{\otimes t}\right)$ and $\operatorname{End}_{\mathfrak{g}}\left(V^{\otimes r} \otimes K_{\lambda_{p q}} \otimes V^{\otimes t}\right)$. Since $\pi_{1}$ is surjectively mapped to $\operatorname{End}_{\mathfrak{g}}\left(V^{\otimes r} \otimes K_{\lambda_{p q}} \otimes V^{\otimes t}\right)$ by [5, IV, Theorem 3.21], we see that $\phi_{1}: \mathscr{B}_{2, r, t} \rightarrow \operatorname{End}_{\mathfrak{g}}\left(M_{p q}^{r t}\right)$ is surjective. Finally, the second assertion follows from the corresponding result for $t=0$ in [5, IV, Theorem 3.21].

\section{Highest Weight VeCtors in $V^{\otimes r} \otimes K_{\lambda_{p q}}$}

The aim of this section is to give a classification of highest weight vectors of $M_{p q}^{r 0}:=V^{\otimes r} \otimes K_{\lambda_{p q}}$ when $r \leq \min \{m, n\}$, where $V$ is the natural representation of $\mathfrak{g}:=\mathfrak{g l}_{m \mid n}$ and $K_{\lambda_{p q}}$ is the Kac-module with highest weight $\lambda_{p q}$ in Example 4.1. This will be done in a few steps. First, by noting that $\mathfrak{g}$-highest weight vectors of $M_{p q}^{r 0}$ is in one to one correspondence with the $\mathfrak{g}_{0}$-highest weight vectors of $V^{\otimes r}$ (cf. [21, Lemmas 5.1-5.2]), we are able to 
reduce the problem to the Lie algebra case. Secondly, since $\mathfrak{g}_{0}=\mathfrak{g l}_{m} \oplus \mathfrak{g l}_{n}$, and $V^{\otimes r}$ can be decomposed as a direct sum of tensor products of natural representations of $\mathfrak{g l}_{m}$ and $\mathfrak{g l}_{n}$, we are able to further simplify the problem to the $\mathfrak{g l}_{m}$ case.

To begin with, we briefly recall the results on a classification of $\mathfrak{g l}_{m}$-highest weight vectors of $V^{\otimes r}$, where $V$ temporarily denotes the natural representation of $\mathfrak{g l}_{m}$ over $\mathbb{C}$. Let $\left\{v_{i} \mid 1 \leq i \leq m\right\}$ be a basis of $V$. Obviously, $V^{\otimes r}$ has a basis $\left\{v_{\mathbf{i}} \mid \mathbf{i} \in I(m \mid 0, r)\right\}$, where

$$
v_{\mathbf{i}}=v_{i_{r}} \otimes v_{i_{r-1}} \otimes \cdots \otimes v_{i_{1}} .
$$

We consider a Cashmir element $\Omega$ in $\mathfrak{g l}_{m}^{\otimes 2}$ with

$$
\Omega=\sum_{1 \leq i, j \leq m} E_{i j} \otimes E_{j i} \in \mathfrak{g l}_{m}^{\otimes 2},
$$

which is a special case of (4.10). Define $\mathbf{s}_{i}=\pi_{i, i+1}(\Omega), 1 \leq i \leq r-1$. Then $(i, i+1) \in \mathfrak{S}_{r}$ acts on $V^{\otimes r}$ via $\mathbf{s}_{i}$. Thus, $V^{\otimes r}$ is a $\left(\mathfrak{g l}_{m}, \mathbb{C S}_{r}\right)$-bimodule such that

$$
v_{\mathbf{i}} w=v_{i_{(r) w^{-1}}} \otimes v_{i_{(r-1) w^{-1}}} \otimes \cdots \otimes v_{i_{(1) w^{-1}}} \text { for any } w \in \mathfrak{S}_{r} .
$$

For example, $v_{i_{3}} \otimes v_{i_{2}} \otimes v_{i_{1}} s_{1} s_{2}=v_{i_{1}} \otimes v_{i_{3}} \otimes v_{i_{2}}$. If $r \leq m$, it is well-known that

$$
\operatorname{End}_{U\left(\mathfrak{g l}_{m}\right)}\left(V^{\otimes r}\right) \cong \mathbb{C S}_{r} .
$$

Definition 5.1. If $\lambda \in \Lambda^{+}(r, m)$, the set of partitions of $r$ with at most $m$ parts, we define $v_{\lambda}=v_{\mathbf{i}_{\lambda}} \in V^{\otimes r}$, where $\mathbf{i}_{\lambda}=\left(1^{\lambda_{1}}, 2^{\lambda_{2}}, \cdots, m^{\lambda_{m}}\right)$ and $k^{\lambda_{k}}$ denotes the sequence $k, k, \cdots, k$ with multiplicity $\lambda_{k}$.

The following result is well-known, and Lemma 5.3 follows from Lemma 5.2

Lemma 5.2. Suppose $\lambda$ and $\mu$ are two compositions of $r$ and $\mu^{\prime}$ is the conjugate of $\mu$, and $x_{\lambda}, y_{\mu^{\prime}}$ are defined in (3.8). Then $x_{\lambda} \mathbb{C S}_{r} y_{\mu^{\prime}}=0$ unless $\lambda \unlhd \mu$.

Lemma 5.3. There is a bijection between the set of dominant weights of $V^{\otimes r}$ and $\Lambda^{+}(r, m)$, the set of partitions of $r$ with at most $m$ parts. Further, the $\mathbb{C}$-space of $\mathfrak{g l}_{\mathfrak{m}}$-highest weight vectors with highest weight $\lambda$ has a basis $\left\{v_{\lambda} w_{\lambda} y_{\lambda^{\prime}} d(\mathfrak{t}) \mid \mathfrak{t} \in \mathcal{T}^{s}\left(\lambda^{\prime}\right)\right\}$.

Now, we turn to construct $\mathfrak{g}$-highest weight vectors of $M_{p q}^{r 0}$. Since $r \leq \min \{m, n\}$, there is a bijection between the set of dominant weights of $M_{p q}^{r 0}$ and $\Lambda_{2}^{+}(r)$. Further, if $\lambda=\left(\lambda^{(1)}, \lambda^{(2)}\right) \in \Lambda_{2}^{+}(r)$, the corresponding dominant weight of $M_{p q}^{r 0}$ is

$$
\bar{\lambda}:=\lambda_{p q}+\tilde{\lambda},
$$

where

$$
\tilde{\lambda}=\left(\lambda_{1}^{(1)}, \cdots, \lambda_{m}^{(1)} \mid \lambda_{1}^{(2)}, \cdots, \lambda_{n}^{(2)}\right) .
$$

For instance, if $\lambda=((3,1),(2,1))$, then $\tilde{\lambda}=(3,1,0, \cdots, 0 \mid 2,1,0, \cdots, 0)$. Recall that $\Omega$ is a Casimir element in $\mathfrak{g}^{\otimes 2}$ given in (4.10). Define operators $s_{i}, x_{1}$ acting on the right of $M_{p q}^{r 0}$ via the following formulae: $s_{i}=\pi_{i+1, i}(\Omega), 1 \leq i \leq r-1$ and $x_{1}=-\pi_{10}(\Omega)$. In this case, $u_{1}=-p$ and $u_{2}=m-q$. We remind that Brundan-Stroppel [5] defined $x_{1}$ via $\pi_{10}(\Omega)$. So, the current $x_{1}$ is $-x_{1}$ in [5]. Recall that $v_{\mathbf{i}} \otimes v_{p q}=v_{i_{r}} \otimes \cdots \otimes v_{i_{2}} \otimes v_{i_{1}} \otimes v_{p q}$ for any $\mathbf{i} \in I(m \mid n, r)$ (cf. (4.7)), and $x_{k}^{\prime}=x_{k}+L_{k}$ with $L_{k}=\sum_{i=1}^{k-1}(i, k)$ (see Lemma 2.7). 
Lemma 5.4. [5, Lemma 3.1] Suppose $\mathbf{i} \in I(m \mid n, r)$, and $1 \leq k \leq r$.

(1) $v_{\mathbf{i}} \otimes v_{p q} x_{k}^{\prime}=-p v_{\mathbf{i}} \otimes v_{p q}$ if $1 \leq i_{k} \leq m$.

(2) $v_{\mathbf{i}} \otimes v_{p q} x_{k}^{\prime}=-q v_{\mathbf{i}} \otimes v_{p q}+\sum_{j=1}^{m}(-1)^{\sum_{l=1}^{k-1}\left[i_{l}\right]} v_{\mathbf{j}} \otimes\left(E_{i_{k}, j} v_{p q}\right)$ if $m+1 \leq i_{k} \leq m+n$, where $\mathbf{j} \in I(m \mid n, r)$ which is obtained from $\mathbf{i}$ by using $j$ instead of $i_{k}$ in $\mathbf{i}$. In particular, the weight of $v_{\mathbf{j}}$ is strictly bigger than that of $v_{\mathbf{i}}$.

Definition 5.5. For $\lambda=\left(\lambda^{(1)}, \lambda^{(2)}\right) \in \Lambda_{2}^{+}(r)$, define $v_{\tilde{\lambda}}=v_{\mathbf{i}}$ with $\mathbf{i}=\left(\mathbf{i}_{\lambda^{(1)}}, \mathbf{i}_{\lambda^{(2)}}\right) \in I(m \mid n, r)$.

For instance, $v_{\tilde{\lambda}}=v_{\mathbf{i}}$ if $\lambda=((3,1),(2,1))$, where $\mathbf{i}=\left(1^{3}, 2,(m+1)^{2}, m+2\right)$.

Definition 5.6. For any $\mathfrak{t} \in \mathcal{T}^{s}\left(\lambda^{\prime}\right)$, we define $v_{\mathfrak{t}}=v_{\tilde{\lambda}} \otimes v_{p q} w_{\lambda} \mathfrak{y}_{\lambda^{\prime}} d(\mathfrak{t})$, where $\mathfrak{y}_{\lambda^{\prime}}$ is given in Definition $3.2(2)$.

Theorem 5.7. Suppose $r \leq \min \{m, n\}$. There is a bijection between the set of dominant weights of $M_{p q}^{r 0}$ and $\Lambda_{2}^{+}(r)$. Further, the $\mathbb{C}$-space $V_{\bar{\lambda}}$ of $\mathfrak{g}$-highest weight vectors of $M_{p q}^{r 0}$ with highest weight $\bar{\lambda}$ has a basis $\left\{v_{\mathfrak{t}} \mid \mathfrak{t} \in \mathcal{T}^{s}\left(\lambda^{\prime}\right)\right\}$.

Proof. The required bijection between $\Lambda_{2}^{+}(r)$ and the set of dominant weights of $M_{p q}^{r 0}$ is the map sending $\lambda$ to $\bar{\lambda}$ defined in (5.3). We claim that each $v_{\mathfrak{t}}$ is killed by $E_{m, m+1}$ and $E_{i, j}$ with $i<j$ and either $i, j \in I_{0}$ or $i, j \in I_{1}$. Since $M_{p q}^{r 0}$ is $\left(\mathfrak{g}, \mathscr{H}_{2, r}\right)$-bimodule, we need only consider the case $d(\mathfrak{t})=1$. In this case, $\mathfrak{t}=\mathfrak{t}^{\lambda^{\prime}}$.

Denote $\left|\lambda^{(1)}\right|=a$. Recall that $w_{\lambda^{(1)}} \in \mathfrak{S}_{a}$ and $w_{\lambda^{(2)}} \in \mathfrak{S}_{r-a}$ such that $\mathfrak{t}^{\lambda^{(i)}} w_{\lambda^{(i)}}=\mathfrak{t}_{\lambda^{(i)}}$ for $i=1,2$. Then

$$
w_{\lambda}=w_{\lambda^{(1)}} w_{\lambda^{(2)}} w_{a}=w_{a} w_{\lambda^{(2)}} w_{\lambda^{(1)}}
$$

By (3.6) and (5.5),

$$
v_{\mathrm{t}}=v_{\tilde{\lambda}} \otimes v_{p q} w_{\lambda^{(1)}} w_{\lambda^{(2)}} y_{\mu^{(1)}} x_{\mu^{(2)}} w_{a} \pi_{r-a}(-p),
$$

where $\mu^{(i)}$ is the conjugate of $\lambda^{(i)}$ for $i=1,2$. By Lemmas 5.25 .3 , $v_{\mathfrak{t}}$ is killed by $E_{i, j}$ with $i<j$ and either $i, j \in I_{0}$ or $i, j \in I_{1}$. Since $E_{m, m+1}$ acts on $M_{p q}^{r 0}$ via $\sum_{i=1}^{r+1} 1^{\otimes i-1} \otimes E_{m, m+1} \otimes 1^{\otimes r+1-i}$, we have $E_{m, m+1} v_{\tilde{\lambda}} \otimes v_{p q}=0$ if $v_{m+1}$ does not occur in $v_{\tilde{\lambda}}$. Otherwise, $\lambda^{(2)} \neq \emptyset$ and $r-a \neq 0$. In this case, up to a sign, $E_{m, m+1} v_{\tilde{\lambda}} \otimes v_{p q}$ is equal to

$$
v_{\mathbf{j}} \otimes v_{p q}\left(1-s_{a+1}+s_{a+1, a+3}+\cdots+(-1)^{b-a} s_{a+1, b+1}\right),
$$

where $b=a+\lambda_{1}^{(2)}-1$ and $v_{\mathbf{j}}$ is obtained from $v_{\tilde{\lambda}}$ by replacing $v_{m+1}$ by $v_{m}$ at the $(a+1)$-th position. Thus, $j_{a+1}=m$. Let

$$
h=\left(1-s_{a+1}+s_{a+1, a+3}+\cdots+(-1)^{b-a} s_{a+1, b+1}\right) w_{\lambda^{(1)}} w_{\lambda^{(2)}} y_{\mu^{(1)}} x_{\mu^{(2)}} .
$$

Then $h \in \mathbb{C S}_{a} \otimes \mathbb{C S}_{r-a}$. By (3.6),$h w_{a}=w_{a} h_{1}$ for some $h_{1} \in \mathbb{C S}_{r-a} \otimes \mathbb{C S}_{a}$. Since $h_{1} \pi_{r-a}(-p)=\pi_{r-a}(-p) h_{1}$, it is enough to prove $v_{\mathbf{j}} \otimes v_{p q} w_{a} \pi_{r-a}(-p)=0$. Up to a sign, $v_{\mathbf{j}} \otimes v_{p q} w_{a}=v_{\mathbf{k}} \otimes v_{p q}$ for some $\mathbf{k}$ such that $v_{k_{1}}=v_{m} \in V_{0}$. Since $r-a \neq 0, x_{1}+p$ is a factor of $\pi_{r-a}(-p)$. By Lemma 5.4 $(1), v_{\mathbf{j}} \otimes v_{p q} w_{a} \pi_{r-a}(-p)=0$. Thus, $v_{\mathfrak{t}}$ is a highest weight vector of $M_{p q}^{r 0}$ if $v_{\mathfrak{t}} \neq 0$.

Note that any vector of $M_{p q}^{r 0}$ can be written as $v=\sum_{b \in B} v_{b} \otimes b$, where $B$ is a basis of $K_{\lambda_{p q}}$ defined in (4.6) and $v_{b} \in V^{\otimes r}$. Following [5], $v_{b}$ is called the b-component of $v$. By Lemma 5.4 (2) (or the arguments in the proof of [6, Corollary 3.3]), the $v_{p q}$-component of 
$v_{\tilde{\lambda}} \otimes v_{p q} w_{a} \pi_{r-a}(-p)$ is $v_{\tilde{\lambda}} w_{a} \prod_{i=1}^{r-a}\left(p-q-L_{i}\right)$. By Lemma 2.7 (3), $\prod_{i=1}^{r-a}\left(p-q-L_{i}\right)$ is a central element in $\mathbb{C S}_{r-a}$, which acts on $v_{\tilde{\lambda}} \otimes v_{p q} w_{\lambda^{(2)}} x_{\mu^{(2)}}$ as scalar $\prod_{i=1}^{r-a}\left(p-q-\operatorname{res}_{t^{\mu}}(2)(i)\right)$, where $\mu=\lambda^{\prime}$ and $\operatorname{res}_{t^{\mu}(2)}(i)$ is $j-l$ if $i$ is in the $l$-th row and $j$-th column of $\mathfrak{t}^{\mu^{(2)}}$. Since $\lambda_{p q}$ is typical (cf. (4.4)), and $r \leq \min \{m, n\}, \prod_{i=1}^{r-a}\left(p-q-\operatorname{res}_{t^{\mu}(2)}(i)\right) \neq 0$. So,

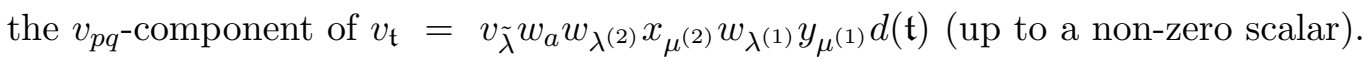

By Lemma 5.3. it is a $\mathfrak{g}_{0}$-highest vector of $V^{\otimes r}$ with highest weight $\tilde{\lambda}$ (cf. (15.4) ), forcing $v_{\mathrm{t}} \neq 0$.

Now, we prove that $\left\{v_{\mathfrak{t}} \mid \mathfrak{t} \in \mathcal{T}^{s}\left(\lambda^{\prime}\right)\right\}$ is $\mathbb{C}$-linear independent. First, consider $V=V_{0} \oplus V_{1}$ as a module for $\mathfrak{g}_{0}=\mathfrak{g l}_{m} \oplus \mathfrak{g l}_{n}$. Then $V^{\otimes r}$ can be decomposed as a direct sum of $V_{i_{1}} \otimes V_{i_{2}} \otimes \cdots \otimes V_{i_{r}}$, where $i_{j} \in\{0,1\}$. As $\mathfrak{g}_{0}$-modules, $V_{i_{1}} \otimes V_{i_{2}} \otimes \cdots \otimes V_{i_{r}} \cong V_{1}^{\otimes r-a} \otimes V_{0}^{a}$ for some non-negative integer $a \leq r$ with $a=\#\left\{i_{j} \mid i_{j}=0\right\}$. The corresponding isomorphism is given by acting a unique element $w$ on the right hand side of $V_{1}^{\otimes r-a} \otimes V_{0}^{a}$, where $w$ is a distinguished right coset representatives of $\mathfrak{S}_{a} \times \mathfrak{S}_{r-a}$ in $\mathfrak{S}_{r}$. By Lemma 5.3, all $\mathfrak{g}_{0}$-highest weight vectors of $V_{i_{1}} \otimes V_{i_{2}} \otimes \cdots \otimes V_{i_{r}}$ with highest weight $\tilde{\lambda}$ are $v_{\tilde{\lambda}} w_{\lambda^{(1)}} y_{\mu^{(1)}} w_{\lambda^{(2)}} x_{\mu^{(2)}} d\left(\mathfrak{t}_{1}\right) d\left(\mathfrak{t}_{2}\right) w$ for all $\mathfrak{t}_{1} \in \mathcal{T}^{s}\left(\mu^{(1)}\right)$ and $\mathfrak{t}_{2} \in \mathcal{T}^{s}\left(\mu^{(2)}\right)$. Therefore, the $\mathbb{C}$-space $V_{\tilde{\lambda}}$ of all $\mathfrak{g}_{0}$-highest weight vectors of $V^{\otimes r}$ with highest weight $\tilde{\lambda}$ has a basis $\left\{v_{\tilde{\lambda}} w_{\lambda^{(2)}} x_{\mu^{(2)}} w_{\lambda^{(1)}} y_{\mu^{(1)}} d(\mathfrak{t}) \mid \mathfrak{t} \in \mathcal{T}^{s}\left(\lambda^{\prime}\right)\right\}$, where $\mu=\lambda^{\prime}$. By (5.6),$\left\{v_{\mathfrak{t}} \mid \mathfrak{t} \in \mathcal{T}^{s}\left(\lambda^{\prime}\right)\right\}$ is $\mathbb{C}$-linear independent. Finally, since there is a one to one correspondence between $\mathfrak{g}$-highest weight vectors of $M_{p q}^{r 0}$ and $\mathfrak{g}_{0}$-highest weight vectors of $V^{\otimes r}$ (cf. [21, Lemmas 5.1-5.2]), and $\operatorname{dim} V_{\tilde{\lambda}}=\#\left\{v_{\mathfrak{t}} \mid \mathfrak{t} \in \mathcal{T}^{s}\left(\lambda^{\prime}\right)\right\}$, one obtains that $\left\{v_{\mathfrak{t}} \mid \mathfrak{t} \in \mathcal{T}^{s}\left(\lambda^{\prime}\right)\right\}$ is a basis of $V_{\bar{\lambda}}$.

In the remaining part of this section, we want to establish the relationship between $V_{\bar{\lambda}}$ with a special cell module of $\mathscr{H}_{2, r}$ with respect to $\lambda \in \Lambda_{2}^{+}(r)$. This result will be needed in section 6 . We go on using $-x_{1}$ instead of $x_{1}$ in [5]. In this case, the current $-p$ and $m-q$ are the same as $p$ and $q$ in [5].

Proposition 5.8. For any $\lambda \in \Lambda_{2}^{+}(r), V_{\bar{\lambda}} \cong \mathfrak{x}_{\lambda} w_{\lambda} \mathfrak{y}_{\lambda^{\prime}} \mathscr{H}_{2, r}$ as right $\mathscr{H}_{2, r}$-modules, where $V_{\bar{\lambda}}$ is defined in Theorem 5.7 .

Proof. By Lemma $3.4(2), S^{\lambda}:=\mathfrak{x}_{\lambda} w_{\lambda} \mathfrak{y}_{\lambda^{\prime}} \mathscr{H}_{2, r}$ has a basis $M=\left\{\mathfrak{x}_{\lambda} w_{\lambda} \mathfrak{y}_{\lambda^{\prime}} d(\mathfrak{t}) \mid \mathfrak{t} \in \mathcal{T}^{s}\left(\lambda^{\prime}\right)\right\}$. It follows from Theorem 5.7 that there is a linear isomorphism $\phi: V_{\bar{\lambda}} \rightarrow S^{\lambda}$ sending $v_{\mathfrak{t}}$ to $\mathfrak{x}_{\lambda} w_{\lambda} \mathfrak{y}_{\lambda^{\prime}} d(\mathfrak{t})$. Obviously, $\phi$ is a right $\mathfrak{S}_{r}$-homomorphism. In order to show that $\phi$ is a right $\mathscr{H}_{2, r}$-homomorphism, it suffices to prove that

$$
\phi\left(v_{\mathfrak{t}} x_{k}\right)=\phi\left(v_{\mathfrak{t}}\right) x_{k} \text { for } 1 \leq k \leq r .
$$

Denote $a=\left|\lambda^{(1)}\right|$. If $1 \leq k \leq r-a$, then $\tilde{\pi}_{\lambda^{\prime}} x_{k}=\pi_{r-a}(m-q) x_{k}=\pi_{r-a}(m-q)\left(-p-L_{k}\right)$. Since $\phi$ is a right $\mathfrak{S}_{r}$-homomorphism, (5.7) holds for $1 \leq k \leq r-a$. If $r-a+1 \leq k \leq r$, then $x_{k}=s_{k, r-a+1} x_{r-a+1} s_{r-a+1, k}-\sum_{j=r-a+1}^{k-1}(j, k)$. By Lemma 3.4(1),

$$
\pi_{\lambda} w_{a} \tilde{\pi}_{\lambda^{\prime}} x_{k}=\pi_{\lambda} w_{a} \tilde{\pi}_{\lambda^{\prime}}\left(-p-\sum_{j=r-a+1}^{k-1}(j, k)\right) .
$$

On the other hand, $\tilde{\pi}_{\lambda^{\prime}} x_{k}=x_{k} \tilde{\pi}_{\lambda^{\prime}}$ and $v_{\tilde{\lambda}} \otimes v_{p q} w_{\lambda^{(1)}} w_{\lambda^{(2)}} y_{\mu^{(1)}} x_{\mu^{(2)}} w_{a}$ is a linear combination of elements $v_{\mathbf{i}} \otimes v_{p q}$, for some $\mathbf{i} \in I(m \mid n, r)$ such that $v_{i_{j}} \in V_{0}$ for all $r-a+1 \leq j \leq r$. By 
Lemma $5.4(1), x_{k}$ acts on $v_{\mathbf{i}} \otimes v_{p q}$ as $-p-L_{k}$. In order to verify (5.7) for $k \geq r-a+1$, by (5.8), it remains to show that

$$
v_{\mathbf{i}} \otimes v_{p q}(i, k) \tilde{\pi}_{\lambda^{\prime}}=0 \text { for all } i, 1 \leq i \leq r-a .
$$

Write $v_{\mathbf{i}} \otimes v_{p q}(i, k)=v_{\mathbf{j}}$ up to a sign. Then $v_{j_{i}} \in V_{0}$ and $v_{\mathbf{j}}(1, i) \tilde{\pi}_{\lambda^{\prime}}=0$ by Lemma 5.4 (1). Since $(1, i) \tilde{\pi}_{\lambda^{\prime}}=\tilde{\pi}_{\lambda^{\prime}}(1, i)$, and $(1, i)$ is invertible, $v_{\mathbf{j}} \tilde{\pi}_{\lambda^{\prime}}=0$, proving (5.9).

Corollary 5.9. Suppose $\lambda \in \Lambda_{2}^{+}(r)$. As right $\mathscr{H}_{2, r}$-modules,

$$
\operatorname{Hom}_{U(\mathfrak{g})}\left(K_{\bar{\lambda}}, M_{p q}^{r 0}\right) \cong \tilde{\Delta}\left(\lambda^{\prime}\right)
$$

where $\tilde{\Delta}\left(\lambda^{\prime}\right)$ is the right cell module defined via the cellular basis of $\mathscr{H}_{2, r}$ in Lemma $3.3(2)$.

Proof. For any $\mathfrak{g}$-highest weight vector $v$ of $M_{p q}^{r 0}$ with highest weight $\bar{\lambda}$, there is a unique $U(\mathfrak{g})$-homomorphism $f_{v}: K_{\bar{\lambda}} \rightarrow \mathbf{U}(\mathfrak{g}) v \subset M_{p q}^{r 0}$ sending $v_{\bar{\lambda}}$ to $v$, where $v_{\bar{\lambda}}$ is the highest weight vector of $K_{\bar{\lambda}}$. Further $f_{v}$ can be considered as a homomorphism in $\operatorname{Hom}_{U(\mathfrak{g})}\left(K_{\bar{\lambda}}, M_{p q}^{r 0}\right)$ by composing an embedding homomorphism.

For any $0 \neq f \in \operatorname{Hom}_{U(\mathfrak{g})}\left(K_{\bar{\lambda}}, M_{p q}^{r 0}\right), f\left(v_{\bar{\lambda}}\right)$ is a highest weight vector of $M_{p q}^{r 0}$. By Theorem 5.7, $f\left(v_{\bar{\lambda}}\right)$ is a linear combination of $v_{\mathfrak{t}}^{\prime}$ 's, for $\mathfrak{t} \in \mathcal{T}^{s}\left(\lambda^{\prime}\right)$. So, $f$ can be written as a linear combination of $f_{v_{\mathfrak{t}}}$ 's. Thus, $\left\{f_{v_{\mathfrak{t}}} \mid \mathfrak{t} \in \mathcal{T}^{s}\left(\lambda^{\prime}\right)\right\}$ is a basis of $\operatorname{Hom}_{U(\mathfrak{g})}\left(K_{\bar{\lambda}}, M_{p q}^{r 0}\right)$. Let $V_{\bar{\lambda}}$ be defined in Theorem 5.7. Then the linear isomorphism $\phi: \operatorname{Hom}_{U(\mathfrak{g})}\left(K_{\bar{\lambda}}, M_{p q}^{r 0}\right) \rightarrow V_{\bar{\lambda}}$ sending $f_{v_{\mathfrak{t}}}$ to $v_{\mathfrak{t}}$ for any $\mathfrak{t} \in \mathcal{T}^{s}\left(\lambda^{\prime}\right)$ is a right $\mathscr{H}_{2, r}$-homomorphism. By Lemma 3.4(5) and Proposition 5.8, $V_{\bar{\lambda}} \cong \tilde{\Delta}\left(\lambda^{\prime}\right)$, proving (5.10).

In the remaining part of this section, we always assume $p-q \leq-m$. If $p-q \geq n$, one can switch roles between $p$ and $q$ (or consider the dual module of $M_{p q}^{r 0}$ ). Without loss of any generality, we assume $p, q \in \mathbb{Z}$.

Let $\lambda \in \Lambda_{2}^{+}(r)$ with $r \leq \min \{m, n\}$. Then $\lambda$ corresponds to a dominant weight $\bar{\lambda}$ defined in (5.3). In particular, $\bar{\emptyset}=\lambda_{p q}$. Following [5, 13, 22], we are going to represent a dominant weight $\bar{\lambda}$ in a unique way by a weight diagram $D_{\lambda}$. First we write (cf. (4.3))

$$
\bar{\lambda}^{\rho}=\bar{\lambda}+\rho=\left(\bar{\lambda}_{1}^{L, \rho}, \ldots, \bar{\lambda}_{m}^{L, \rho} \mid \bar{\lambda}_{1}^{R, \rho}, \ldots, \bar{\lambda}_{n}^{R, \rho}\right) .
$$

Denote

$$
\begin{aligned}
S(\lambda)_{\mathrm{L}} & =\left\{\bar{\lambda}_{i}^{L, \rho} \mid i=1, \ldots, m\right\}, & & S(\lambda)_{\mathrm{R}}=\left\{-\bar{\lambda}_{j}^{R, \rho} \mid j=1, \ldots, n\right\}, \\
S(\lambda) & =S(\lambda)_{\mathrm{L}} \cup S(\lambda)_{\mathrm{R}}, & & S(\lambda)_{\mathrm{B}}=S(\lambda)_{\mathrm{L}} \cap S(\lambda)_{\mathrm{R}} .
\end{aligned}
$$

Definition 5.10. The weight diagram $D_{\lambda}$ associated with the dominant weight $\bar{\lambda}$ is a line with vertices indexed by $\mathbb{Z}$ such that each vertex $i$ is associated with a symbol $D_{\lambda}^{i}=\emptyset,<,>$ or $\times$ according to whether $i \notin S(\lambda), i \in S(\lambda)_{\mathrm{R}} \backslash S(\lambda)_{\mathrm{B}}, i \in S(\lambda)_{\mathrm{L}} \backslash S(\lambda)_{\mathrm{B}}$ or $i \in S(\lambda)_{\mathrm{B}}$.

For example, if $p, q \in \mathbb{Z}$ with $p \leq q-m$, then the weight diagram $D_{\emptyset}$ of $\bar{\emptyset}=\lambda_{p q}$ is given by

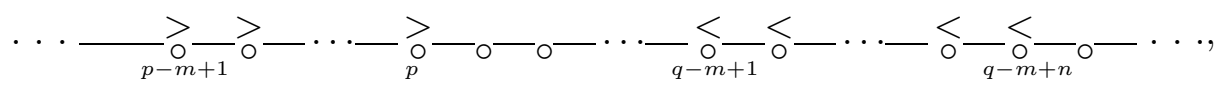

where, for simplicity, we have associated vertex $i$ with nothing if $D_{\lambda}^{i}=\emptyset$. Note that $\sharp S(\emptyset)_{\mathrm{B}}=0$, i.e., $\lambda_{p q}$ is typical. 
Definition 5.11. Let $\bar{\lambda}$ be as in (5.3), where $\lambda \in \Lambda_{2}^{+}(r)$.

(1) Let $\bar{\lambda}^{\text {top }}$ be the unique dominant weight such that $L_{\bar{\lambda}}$ is the simple submodule of the Kac-module $K_{\bar{\lambda}^{\text {top }}}$. Then $\bar{\lambda}^{\text {top }}$ is obtained from $\bar{\lambda}$ via the unique longest right path (cf. [22, Definition 5.2], 24, Conjecture 4.4]) or via a raising operator (cf. 4]). For example, if $D_{\lambda}$ is given by

$$
\cdots-0-\stackrel{\times}{1}-\stackrel{\times}{2}-3-\stackrel{\times}{4}-\underset{5}{5}-6-\stackrel{\times}{7}-\stackrel{<}{8}-9-\underset{<}{10}-11-\cdots
$$

then the weight diagram $D_{\lambda^{\text {top }}}$ of $\bar{\lambda}^{\text {top }}$ is given by

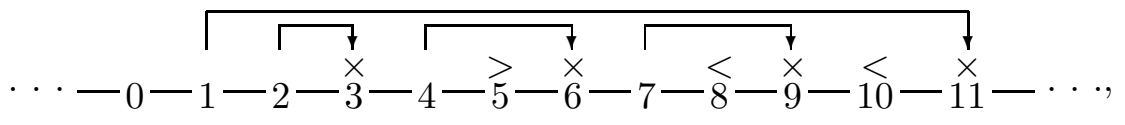

where the $\times$ 's at vertices $9,6,3,11$ in (5.14) are respectively obtained from the $\times$ 's at vertices $7,4,2,1$ in (5.13) (thus every symbol " $x$ " is always moved to the unique empty place at its right side which is closest to it, under the rule that the rightmost " $\times$ " should be moved first, as indicated in (5.14)). Alternatively, $\bar{\lambda}$ is obtained from $\bar{\lambda}^{\text {top }}$ via the unique longest left path.

(2) Let $\lambda^{\text {top }} \in \Lambda_{2}^{+}(r)$ be the unique bipartition such that $\bar{\lambda}^{\text {top }}=\lambda_{p q}+\tilde{\lambda}^{\text {top }}$ (cf. (5.4) and (5.3) ).

Write $p=q-m-k$ for some $k \in \mathbb{N}$. If $\mu=\left(\left(\mu_{1}^{L}, \ldots, \mu_{m}^{L}\right),\left(\mu_{1}^{R}, \ldots, \mu_{n}^{R}\right)\right) \in \Lambda_{2}^{+}(r)$, then $\mu^{\prime}$ is Kleshchev with respect to $u_{1}=-p, u_{2}=m-q$ (cf. (3.15) $)$ if and only if

$$
\mu_{i}^{L} \geq \mu_{i}^{R}-k \text { for all possible } i \text {. }
$$

Following [5, IV], we denote $I_{p q}^{+}=\{p-m+1, p-m+2, \ldots, q-m+n\}$. For any $\lambda \in \Lambda_{2}^{+}(r)$ and any $j \in I_{p q}^{+}$, set

$$
\begin{aligned}
& I_{\geq j}^{\emptyset}(\lambda)=\mathbb{Z}^{\geq j} \cap\left(I_{p q}^{+} \backslash S(\lambda) \cap I_{p q}^{+}\right), \\
& I_{\leq j}^{\emptyset}(\lambda)=\mathbb{Z}^{\leq j} \cap\left(I_{p q}^{+} \backslash S(\lambda) \cap I_{p q}^{+}\right), \\
& I_{\geq j}^{\times}(\lambda)=\mathbb{Z}^{\geq j} \cap\left(I_{p q}^{+} \cap S(\lambda)_{\mathrm{B}},\right. \\
& I_{\leq j}^{\times}(\lambda)=\mathbb{Z}^{\leq j} \cap\left(I_{p q}^{+} \cap S(\lambda)_{\mathrm{B}} .\right.
\end{aligned}
$$

In terms of the above notations, Brundan and Stroppel [5, IV, Lemma 2.6] have proved that the indecomposable tilting module $T_{\bar{\lambda}}$ is a direct summand of $M_{p q}^{r 0}$ if

$$
S(\lambda) \subset I_{p q}^{+} \text {and } \# I_{\geq j}^{\emptyset}(\lambda) \geq \# I_{\geq j}^{\times}(\lambda) \text { for all } j \in I_{p q}^{+} .
$$

These two conditions on bipartition $\lambda$ (or weight $\bar{\lambda}$ ) are equivalent to the following conditions on $\lambda^{\text {top }}$ (which can be seen from (5.13)-(5.14) in case $I_{p q}^{+}=\{1,2, \ldots, 11\}$ ):

$$
S\left(\lambda^{\text {top }}\right) \subset I_{p q}^{+} \text {and } \# I_{\leq j}^{\emptyset}\left(\lambda^{\text {top }}\right) \geq \# I_{\leq j}^{\times}\left(\lambda^{\text {top }}\right) \text { for all } j \in I_{p q}^{+} \text {. }
$$

Lemma 5.12. Let $\mu \in \Lambda_{2}^{+}(r)$ such that $\mu^{\prime}$ is Kleshchev with respect to $u_{1}=-p, u_{2}=m-q$, where $p=q-m-k$ with $k \in \mathbb{N}$. Then

$$
S(\mu) \subset I_{p q}^{+} \text {and } \# I_{\leq j}^{\emptyset}(\mu) \geq \# I_{\leq j}^{\times}(\mu) \text { for all } j \in I_{p q}^{+} \text {. }
$$


Proof. We have (cf. (4.3))

$$
\lambda_{p q}+\rho=(q-m-k, \ldots, q-2 m-k+1 \mid-q+m-1, \ldots,-q+m-n) .
$$

Thus for $i=1, \ldots, m$, we have (cf. (5.11) ) $\bar{\mu}_{i}^{L, \rho}=\mu_{i}+q-m-k \geq q-2 m-k+1$ and $\bar{\mu}_{i}^{L, \rho} \leq q+n-m\left(\right.$ as $\left.\mu_{i} \leq r \leq n\right)$, i.e., $\bar{\mu}_{i}^{L, \rho} \in I_{p q}^{+}$. Similarly, $-\bar{\mu}_{j}^{R, \rho} \in I_{p q}^{+}$for $j=1, \ldots, n$. Hence, $S(\mu) \subset I_{p q}^{+}$.

To prove the other assertion of (5.22), note that the weight diagram $D_{\mu}$ of $\bar{\mu}$ is obtained from $D_{\emptyset}$ (cf. (5.12) ) by moving the ">" at vertex $p-i$ for all $i$ with $0 \leq i \leq m-1$ to its right side to vertex $p-i+\mu_{i+1}^{L}$, and moving the "<" at vertex $q-m+j$ for all $j$ with $1 \leq j \leq n$ to its left side to vertex $q-m+j-\mu_{j}^{R}$ (if " $<$ " meets " $>$ " at the destination vertex, then two symbols " $<$ " and " $>$ " are combined to become the symbol " $\times$ "). Since $\mu^{\prime}$ is Kleshchev, condition (5.15) shows that in order to produce a " $\times$ " at some vertex $i$ of $D_{\mu}$, a " $>$ " at some vertex $j$ with $j<i$ must be moved to vertex $i$, i.e., an " $\emptyset$ " must appear in some vertex $j^{\prime}$ with $j^{\prime} \leq j<i$, i.e., (5.21) holds.

Corollary 5.13. Suppose $\lambda \in \Lambda_{2}^{+}(r)$ such that $\left(\lambda^{\text {top }}\right)^{\prime}$ is Kleshchev, where $\left(\lambda^{\text {top }}\right)^{\prime}$ is the conjugate of $\lambda^{\text {top }} \in \Lambda_{2}^{+}(r)$. Then $T_{\bar{\lambda}}$ is a direct summand of $M_{p q}^{r 0}$. Further, any indecomposable direct summand of $M_{p q}^{r 0}$ is of form $T_{\bar{\lambda}}$ for some $\lambda \in \Lambda_{2}^{+}(r)$ such that $\left(\lambda^{\text {top }}\right)^{\prime}$ is Kleshchev.

Proof. The first assertion follows from [5, IV, Lemma 2.6] and Lemma [5.12. To prove the last assertion, since $r \leq \min \{m, n\}$, by Theorem 4.5, $\operatorname{End}_{U\left(\mathfrak{g l}_{m \mid n}\right)}\left(M_{p q}^{r 0}\right) \cong \mathscr{H}_{2, r}$. So, the number of non-isomorphic indecomposable direct summands of $\mathfrak{g l}_{m \mid n}$-module $M_{p q}^{r 0}$ is equal to that of non-isomorphic irreducible $\mathscr{H}_{2, r}$-modules, which is equal to the number of Kleshchev bipartitions in $\Lambda_{2}^{+}(r)$. Now, everything is clear.

Corollary 5.14. Suppose $\lambda \in \Lambda_{2}^{+}(r)$ such that $\left(\lambda^{\text {top }}\right)^{\prime}$ is Kleshchev. As right $\mathscr{H}_{2, r}$-modules,

$$
\operatorname{Hom}_{U(\mathfrak{g})}\left(T_{\bar{\lambda}}, M_{p q}^{r 0}\right) \cong P^{\left(\lambda^{\mathrm{top}}\right)^{\prime}},
$$

where $P^{\left(\lambda^{\mathrm{top}}\right)^{\prime}}$ is the projective cover of $D^{\left(\lambda^{\mathrm{top}}\right)^{\prime}}$ which is the simple head of $\tilde{\Delta}\left(\left(\lambda^{\mathrm{top}}\right)^{\prime}\right)$.

Proof. Since $r \leq \min \{m, n\}$ and $\left(\lambda^{\text {top }}\right)^{\prime}$ is Kleshchev, by Corollary 5.13 , $T_{\bar{\lambda}}$ is a direct summand of $M_{p q}^{r 0}$, forcing $0 \neq \operatorname{Hom}_{U(\mathfrak{g})}\left(T_{\bar{\lambda}}, M_{p q}^{r 0}\right)$ to be a direct summand of $\mathscr{H}_{2, r}$. We claim that $\operatorname{Hom}_{U(\mathfrak{g})}\left(T_{\bar{\lambda}}, M_{p q}^{r 0}\right)$ is indecomposable. If not, then the number of indecomposable direct summands of the right $\mathscr{H}_{2, r}$-module $\mathscr{H}_{2, r}$ is strictly bigger than $\sum_{\bar{\lambda}} \ell_{\bar{\lambda}}$ if we write $M_{p q}^{r 0}$ as $M_{p q}^{r 0}=\oplus_{\bar{\lambda}} T_{\bar{\lambda}}^{\oplus \ell_{\bar{\lambda}}}$ with $\ell_{\bar{\lambda}} \neq 0$.

On the other hand, since $M_{p q}^{r 0}$ is a right $\mathscr{H}_{2, r}$-module, we can consider the right exact functor $\mathfrak{F}:=M_{p q}^{r 0} \otimes_{\mathscr{H}_{2, r}}$ ? from the category of left $\mathscr{H}_{2, r}$-modules to the category of left $U(\mathfrak{g})$ modules. We have an epimorphism from $\mathfrak{F}\left(P^{\mu}\right)$ to $\mathfrak{F}(\tilde{\Delta}(\mu))$, where $P^{\mu}$ is any principal indecomposable left $\mathscr{H}_{2, r}$-module and $\tilde{\Delta}(\mu)$ temporally denotes the left cell module of $\mathscr{H}_{2, r}$ defined via the cellular basis of $\mathscr{H}_{2, r}$ in Lemma $3.3(1)$ with the simple head $D^{\mu}$. By Lemma 3.4(5) and Theorem 4.5, $\mathfrak{F}(\tilde{\Delta}(\mu)) \neq 0$, forcing $\mathfrak{F}\left(P^{\mu}\right) \neq 0$. So, $\mathfrak{F}\left(P^{\mu}\right)$ is a direct sum of indecomposable direct summand of $U(\mathfrak{g})$-module $M_{p q}^{r 0}$. In particular, $\sum_{\bar{\lambda}} \ell_{\bar{\lambda}}$ is no less than the number of indecomposable direct summands of left $\mathscr{H}_{2, r}$-module $\mathscr{H}_{2, r}$. This is a contradiction since the 
number of indecomposable direct summands of left $\mathscr{H}_{2, r}$-module $\mathscr{H}_{2, r}$ is equal to that of indecomposable direct summands of right $\mathscr{H}_{2, r}$-module $\mathscr{H}_{2, r}$. So, $\mathfrak{F}\left(T_{\bar{\lambda}}\right)$ is a principal indecomposable right $\mathscr{H}_{2, r}$-module. Since $K_{\lambda^{\text {top }}} \hookrightarrow T_{\bar{\lambda}}, \operatorname{Hom}_{U(\mathfrak{g})}\left(T_{\bar{\lambda}}, M_{p q}^{r 0}\right) \rightarrow \operatorname{Hom}_{U(\mathfrak{g})}\left(K_{\bar{\lambda}^{\text {top }}}, M_{p q}^{r 0}\right)$. By Corollary 5.9, $\operatorname{Hom}_{U(\mathfrak{g})}\left(K_{\bar{\lambda}^{\text {top }}}, M_{p q}^{r 0}\right) \cong \tilde{\Delta}\left(\left(\lambda^{\text {top }}\right)^{\prime}\right)$. Since $\operatorname{Hom}_{U(\mathfrak{g})}\left(T_{\bar{\lambda}}, M_{p q}^{r 0}\right)$ is a principal indecomposable right $\mathscr{H}_{2, r}$-module, it implies that $\tilde{\Delta}\left(\left(\lambda^{\text {top }}\right)^{\prime}\right)$ has the unique simple head, denoted by $D^{\left(\lambda^{\mathrm{top}}\right)^{\prime}}$. Thus, $\operatorname{Hom}_{\mathfrak{g}}\left(T_{\bar{\lambda}}, M_{p q}^{r 0}\right) \cong P^{\left(\lambda^{\mathrm{top}}\right)^{\prime}}$.

Brundan-Stroppel have already proved that decomposition numbers of $\mathscr{H}_{2, r}$ arising from super Schur-Weyl duality in [5] can be determined by the multiplicity of Kac-modules in indecomposable tilting modules appearing in $M_{p q}^{r 0}$. This result can also be seen via the exact functor $\operatorname{Hom}_{U(\mathfrak{g})}\left(?, M_{p q}^{r 0}\right)$.

\section{Highest weight vectors in $M_{p q}^{r t}$}

In this section, we classify $\mathfrak{g}$-highest weight vectors of $\mathfrak{g l}_{m \mid n}$-module $M_{p q}^{r t}$ over $\mathbb{C}$. As an application, we set up explicit relationship between Kac (resp. indecomposable tilting) modules of $\mathfrak{g}$ and cell (resp. principal indecomposable) modules of $\mathscr{B}_{2, r, t}$. This gives us an efficient way to calculate decomposition numbers of $\mathscr{B}_{2, r, t}$. Throughout, assume $r, t \in \mathbb{Z}^{>0}$ such that $r+t \leq \min \{m, n\}$. The case $t=0$ has been dealt with in section 5 . By symmetry, one can also classify highest weight vectors of $M_{p q}^{0 t}$ via those in section 5 . The following result, which is the counterpart of Lemma 5.4, can be verified directly.

Lemma 6.1. Suppose $\mathbf{i} \in I(m \mid n, r), \mathbf{j} \in \bar{I}(m \mid n, t)$ (cf. (4.7)) and $1 \leq k \leq t$.

(1) $v_{\mathbf{i}} \otimes v_{p q} \otimes \bar{v}_{\mathbf{j}} \bar{x}_{k}^{\prime}=q v_{\mathbf{i}} \otimes v_{p q} \otimes \bar{v}_{\mathbf{j}}$ if $1+m \leq j_{k} \leq m+n$.

(2) $v_{\mathbf{i}} \otimes v_{p q} \otimes \bar{v}_{\mathbf{j}} \bar{x}_{k}^{\prime}=p v_{\mathbf{i}} \otimes v_{p q} \otimes \bar{v}_{\mathbf{j}}+\sum_{j=m+1}^{m+n}(-1)^{\sum_{l=1}^{k-1}\left[j_{l}\right]} v_{\mathbf{i}} \otimes\left(E_{j j_{k}} v_{p q}\right) \otimes \bar{v}_{\ell}$ if $1 \leq j_{k} \leq m$, where $\ell \in \bar{I}(m \mid n, t)$ which is obtained from $\mathbf{j}$ by using $j$ instead of $j_{k}$ in $\mathbf{j}$. In particular, the weight of $\bar{v}_{\ell}$ is strictly bigger than that of $\bar{v}_{\mathbf{j}}$.

For any integral weight $\xi=\left(\xi_{1}, \ldots, \xi_{m} \mid \xi_{m+1}, \ldots, \xi_{m+n}\right)$ of $\mathfrak{g}$, let

$$
\xi^{L}=\left(\xi_{1}^{L}, \ldots, \xi_{m}^{L}\right)=\left(\xi_{1}, \ldots, \xi_{m}\right), \text { and } \xi^{R}=\left(\xi_{1}^{R}, \ldots, \xi_{m}^{R}\right)=\left(\xi_{m+1}, \ldots, \xi_{m+n}\right) .
$$

We define two bicompositions $\mu, \nu$ such that all $\mu_{i}^{(1)}, \mu_{j}^{(2)}, \nu_{i}^{(1)}, \nu_{j}^{(2)}$ are zero except that

(1) for $1 \leq i \leq m, \mu_{i}^{(1)}=\xi_{i}^{L}$ if $\xi_{i}^{L}>0$ or $\nu_{m-i+1}^{(1)}=-\xi_{i}^{L}$ if $\xi_{i}^{L}<0$.

(2) for $1 \leq j \leq n, \mu_{j}^{(2)}=\xi_{j}^{R}$ if $\xi_{j}^{R}>0$ or $\nu_{n-j+1}^{(2)}=-\xi_{j}^{R}$ if $\xi_{j}^{R}<0$.

Then both $\mu$ and $\nu$ correspond to integral weights of $\mathfrak{g}$. In particular, $\xi=\mu-\hat{\nu}$ with

$$
\hat{\nu}=\left(\nu_{m}^{(1)}, \ldots, \nu_{1}^{(1)} \mid \nu_{n}^{(2)}, \ldots, \nu_{1}^{(2)}\right) \in \mathfrak{h}^{*} .
$$

Conversely, if $\mu$ and $\nu$ are two bicompositions, then $\xi=\mu-\hat{\nu}$ is a integral weight of $\mathfrak{g}$. For instance, if $\xi=(r-4,1,0, \cdots, 0,-1,-(t-5) \mid 2,1,0, \cdots, 0,-1,-3)$, then $\mu=((r-4,1),(2,1))$ and $\nu=((t-5,1),(3,1))$ such that $\xi=\mu-\hat{\nu}$.

Definition 6.2. For any $\lambda=(f, \mu, \nu) \in \Lambda_{2, r, t}$, let $\bar{\lambda}:=\lambda_{p q}+\mu-\hat{\nu}$ and $\tilde{\lambda}:=\mu-\hat{\nu}$. Since $r+t \leq \min \{m, n\}$, both $\mu$ and $\nu$ correspond to integral weights of $\mathfrak{g}$ as above such that

$$
\mu_{i} \nu_{m+1-i}=0 \text { for } 1 \leq i \leq m \text { and } \mu_{m+j} \nu_{m+n+1-j}=0 \text { for } 1 \leq j \leq n,
$$


Lemma 6.3. For any $\mathfrak{g}$-highest weight $\Lambda$ of $M_{p q}^{r t}$, there is a unique triple $\lambda=(f, \mu, \nu) \in \Lambda_{2, r, t}$ such that $\Lambda=\bar{\lambda}$.

Proof. By [19, Lemma 5.20], $\Lambda=\lambda_{p q}+\eta-\zeta$ for some bicompositions $\eta$ and $\zeta$ of sizes $r$ and $t$ respectively. For $i \in I$, let $\xi_{i}=\min \left\{\eta_{i}, \zeta_{i}\right\}$ and $f=\sum_{i \in I} \xi_{i}$. Then we obtain a weight $\xi$, and two bicompositions $\mu:=\eta-\xi$ and $\gamma:=\zeta-\xi$ such that $|\mu|=r-f,|\gamma|=t-f$ and $\Lambda=\lambda_{p q}+\mu-\gamma$. Set $\nu=\hat{\gamma}$, then $\Lambda=\bar{\lambda}$ and (6.2) is satisfied by definition of $\xi$. Since $\Lambda$ is dominant, $\mu, \nu$ must be bipartitions. Thus $\Lambda$ corresponds to $\lambda=(f, \mu, \nu) \in \Lambda_{2, r, t}$. Such a $\lambda$ is unique.

Definition 6.4. For each $\lambda=(f, \mu, \nu) \in \Lambda_{2, r, t}$, denote $v_{\lambda}=v_{\mathbf{i}} \otimes v_{p q} \otimes v_{\mathbf{j}}$, where such that

$$
\mathbf{i}=(\mathbf{i}_{\mu^{(1)}}, \mathbf{i}_{\mu^{(2)}}, \underbrace{1, \cdots, 1}_{f}) \in I(m \mid n, r) \text {, and } \mathbf{j}=(\mathbf{j}_{\nu^{(2)}}, \mathbf{j}_{\nu^{(1)}}, \underbrace{1, \cdots, 1}_{f}) \in \bar{I}(m \mid n, t),
$$

(1) $\mathbf{j}_{\nu^{(2)}}$ is obtained from $\mathbf{i}_{\nu^{(2)}}$ by using $m+n-i+1$ instead of $i$ for $1 \leq i \leq n$,

(2) $\mathbf{j}_{\nu^{(1)}}$ is obtained from $\mathbf{i}_{\nu^{(1)}}$ by using $m-i+1$ instead of $i$ for $1 \leq i \leq m$.

For instance, if $\lambda=(1, \mu, \nu) \in \Lambda_{2,8,10}$ with $\mu=((3,1),(2,1))$ and $\nu=((4,1),(3,1))$, then $\mathbf{i}=\left(1^{3}, 2,(m+1)^{2},(m+2), 1\right)$ and $\mathbf{j}=\left((m+n)^{3},(m+n-1), m^{4},(m-1), 1\right)$. Thus,

$$
v_{\lambda}=v_{1} \otimes v_{m+2} \otimes v_{m+1}^{\otimes 2} \otimes v_{2} \otimes v_{1}^{\otimes 3} \otimes v_{p q} \otimes \bar{v}_{m+n}^{\otimes 3} \otimes \bar{v}_{m+n-1} \otimes \bar{v}_{m}^{\otimes 4} \otimes \bar{v}_{m-1} \otimes \bar{v}_{1} .
$$

Definition 6.5. For any $(f, \mu, \nu) \in \Lambda_{2, r, t}$, define

(1) $w_{\mu, \nu}=w_{\mu} w_{\nu^{o}}$, with $\nu^{o}=\left(\nu^{(2)}, \nu^{(1)}\right), w_{\mu}=d\left(\mathfrak{t}_{\mu}\right) \in \mathfrak{S}_{r-f}$ and $w_{\nu^{o}}=d\left(\mathfrak{t}_{\nu^{o}}\right) \in \overline{\mathfrak{S}}_{t-f}$,

(2) $v_{\lambda, \mathfrak{t}, d, \kappa_{d}}=v_{\lambda} \mathfrak{e}^{f} w_{\mu, \nu} \mathfrak{y}_{\mu^{\prime}} \overline{\mathfrak{y}}_{\left(\nu^{o}\right)^{\prime}} d(\mathfrak{t}) d x^{\kappa_{d}}, \mathfrak{t} \in \mathcal{T}^{s}\left(\mu^{\prime}\right) \times \mathcal{T}^{s}\left(\left(\nu^{o}\right)^{\prime}\right), d \in \mathcal{D}_{r, t}^{f}$ and $\kappa_{d} \in \mathbf{N}_{f}$.

Theorem 6.6. Suppose $r+t \leq \min \{m, n\}$.

(1) There is a bijection between the set of dominant weights of $M_{p q}^{r t}$ and $\Lambda_{2, r, t}$.

(2) If $\lambda=(f, \mu, \nu) \in \Lambda_{2, r, t}$, then $V_{\bar{\lambda}}$, the $\mathbb{C}$-space of all $\mathfrak{g}$-highest weight vectors of $M_{p q}^{r t}$ with highest weight $\bar{\lambda}$, has a basis $S:=\left\{v_{\lambda, \mathfrak{t}, d, \kappa_{d}} \mid \mathfrak{t} \in \mathcal{T}^{s}\left(\mu^{\prime}\right) \times \mathcal{T}^{s}\left(\left(\nu^{o}\right)^{\prime}\right), d \in \mathcal{D}_{r, t}^{f}, \kappa_{d} \in \mathbf{N}_{f}\right\}$.

Proof. Obviously, (1) follows from Lemma 6.2. To obtain (2), we prove that for each $\lambda=(f, \mu, \nu) \in \Lambda_{2, r, t}, V_{\bar{\lambda}}$ has the required basis in the case either $f=0$ or $f>0$.

Case 1: $f=0$.

By Definition 6.5, $v_{\lambda, \mathfrak{t}, d, \kappa_{d}}=v_{\mathbf{i}} \otimes v_{p q} \otimes \bar{v}_{\mathbf{j}} w_{\mu} \mathfrak{y}_{\mu^{\prime}} d\left(\mathfrak{t}_{1}\right) w_{\nu^{\circ}} \overline{\mathfrak{y}}_{\left(\nu^{o}\right)^{\prime}} d\left(\mathfrak{t}_{2}\right)$, where $\mathbf{i}, \mathbf{j}$ are defined in Definition 6.4. By Theorem 5.7, $v_{\mathbf{i}} \otimes v_{p q} \otimes \bar{v}_{\mathbf{j}} w_{\mu} \mathfrak{y}_{\mu^{\prime}} d\left(\mathfrak{t}_{1}\right)$ can be regarded as a $\mathfrak{g}$-highest weight vector of $M_{p q}^{r 0}$. Similarly, $v_{\mathbf{i}} \otimes v_{p q} \otimes \overline{\mathbf{v}}_{\mathbf{j}} w_{\nu^{o}} \overline{\mathfrak{y}}_{\left(\nu^{o}\right)^{\prime}} d\left(\mathfrak{t}_{2}\right)$ can be regarded as a $\mathfrak{g}$-highest weight vector of $M_{p q}^{0 t}$. Thus, $v_{\lambda, \mathfrak{t}, d, \kappa_{d}}$ is a $\mathfrak{g}$-highest weight vector of $M_{p q}^{r t}$. The last assertion follows from arguments on counting the dimensions of $V_{\bar{\lambda}}$ and that of $\mathfrak{g}_{0}$-highest weight vectors of $V^{r t}:=V^{\otimes r} \otimes W^{\otimes t}$ with highest weight $\mu-\hat{\nu}$.

Case 2: $f>0$.

For any $i \in I$,

$$
v_{i} \otimes \bar{v}_{i} e_{1}=(-1)^{[i]} \sum_{j \in I} v_{j} \otimes \bar{v}_{j}
$$


Thus $v_{i} \otimes \bar{v}_{i} e_{1}$ is unique up to a sign for different $i$ 's. Since $M_{p q}^{r t}$ is a $\left(\mathfrak{g}, \mathscr{B}_{2, r, t}\right)$-bimodule, we can switch $v_{i_{r-k}}$ and $\bar{v}_{j_{t-k}}$ in $v_{\lambda}$ with $i_{r-k}=j_{t-k}$ to $v_{o}$ and $\bar{v}_{o}$ for any fixed $o, 1 \leq o \leq m+n$ simultaneously when we consider the action of $E_{j, \ell}$ on $i_{r-k}$-th (resp. $j_{t-k}$-th) tensor factor of $v_{\lambda, t, d, \kappa_{d}}$ for $0 \leq k \leq f-1$. Let

$$
v_{\mathfrak{t}}:=v_{i_{r-f}} \otimes \cdots v_{i_{1}} \otimes v_{p q} \otimes \bar{v}_{j_{1}} \otimes \cdots \bar{v}_{j_{t-f}} w_{\mu, \nu} x_{\alpha^{(2)}} y_{\alpha^{(1)}} y_{\beta^{(1)}} x_{\beta^{(2)}} \pi_{r-f-a}(-p) \pi_{b}(q) d(\mathfrak{t}),
$$

where $\alpha^{(i)}\left(\operatorname{resp} . \beta^{(i)}\right)$ is the conjugate of $\mu^{(i)}\left(\operatorname{resp} . \nu^{(i)}\right), i=1,2$. Applying Theorem 5.7 to both $V^{\otimes r-f} \otimes K_{\lambda_{p q}}$ and $K_{\lambda_{p q}} \otimes W^{\otimes t-f}$ yields $E_{j, \ell} v_{\mathfrak{t}}=0$. So, $E_{j, \ell} v_{\lambda, t, d, \kappa_{d}}=0$ for any $j<\ell$.

We claim that $S$ is linear independent, where $S$ is given in (2). If so, each $v_{\lambda, \mathfrak{t}, d, \kappa_{d}} \neq 0$, forcing $v_{\lambda, \mathfrak{t}, d, \kappa_{d}}$ to be a $\mathfrak{g}$-highest weight vector of $M_{p q}^{r t}$ with highest weight $\bar{\lambda}$.

Suppose $\mathbf{i} \in I\left(m \mid n, r_{1}-1\right)$ and $\mathbf{j} \in \bar{I}\left(m \mid n, t_{1}-1\right)$ with $r_{1} \leq r$ and $t_{1} \leq t$ such that there are at least some $k_{0} \in I_{0}$ and $\ell_{0} \in I_{1}$ satisfying $k_{0}, \ell_{0} \notin\left\{i_{l}, j_{o}\right\}$ for all possible $i, o$ 's. We consider $\sum_{k \in I} v_{k} \otimes v_{\mathbf{i}} \otimes v \otimes v_{\mathbf{j}} \otimes \bar{v}_{k} \in M_{p q}^{r_{1}, t_{1}}$, where $v \in B$ is a basis element of $K_{\lambda_{p q}}$ in (4.6). Since $x_{r_{1}}^{\prime}=x_{r_{1}}+L_{r_{1}}$ and $x_{r_{1}}^{\prime}$ acts on $M_{p q}^{r_{1}, t_{1}}$ as $-\pi_{r_{1}, 0}(\Omega)$, where $\Omega$ is given in (4.10), we have

$$
\begin{gathered}
\sum_{k \in I} v_{k} \otimes v_{\mathbf{i}} \otimes v \otimes v_{\mathbf{j}} \otimes \bar{v}_{k}\left(x_{r_{1}}+L_{r_{1}}\right)=-\pi_{r_{1}, 0}(\Omega) \sum_{k \in I} v_{k} \otimes v_{\mathbf{i}} \otimes v \otimes v_{\mathbf{j}} \otimes \bar{v}_{k} \\
=-\sum_{k, i \in I}(-1)^{[k]+([k]+[i])([k]+[\mathbf{i}])} v_{i} \otimes v_{\mathbf{i}} \otimes E_{k, i} v \otimes \bar{v}_{\mathbf{j}} \otimes \bar{v}_{k},
\end{gathered}
$$

where $[\mathbf{i}]=\sum_{j=1}^{r_{1}-1}\left[i_{j}\right]$. So, up to some scalar $a, \sum_{k=1}^{m+n} v_{k} \otimes v_{\mathbf{i}} \otimes v \otimes v_{\mathbf{j}} \otimes \bar{v}_{k} x_{r_{1}}$ contains the unique term $v_{k_{0}} \otimes v_{\mathbf{i}} \otimes v \otimes \bar{v}_{\mathbf{j}} \otimes \bar{v}_{k_{0}}$. In particular, if $v \neq v_{p q}, \sum_{k \in I} v_{k} \otimes v_{\mathbf{i}} \otimes v \otimes v_{\mathbf{j}} \otimes \bar{v}_{k} x_{r_{1}}$ does not contribute terms with form $v_{k_{0}} \otimes v_{\mathbf{i}^{\prime}} \otimes v_{p q} \otimes v_{\mathbf{j}^{\prime}} \otimes \bar{v}_{k_{0}}$ for all possible $\mathbf{i}^{\prime}$ and $\mathbf{j}^{\prime}$. If $v=v_{p q}$, by Lemma 5.4, the previous scalar is $-p$. Similarly, the coefficient of $v_{\ell_{0}} \otimes v_{\mathbf{i}} \otimes v_{p q} \otimes v_{\mathbf{j}} \otimes \bar{v}_{\ell_{0}}$ in the expression of $\sum_{k \in I} v_{k} \otimes v_{\mathbf{i}} \otimes v \otimes v_{\mathbf{j}} \otimes \bar{v}_{k} x_{r_{1}}$ is $-q$. Assume

$$
c \sum_{k \in I} v_{k} \otimes v_{\mathbf{i}} \otimes v_{p q} \otimes v_{\mathbf{j}} \otimes \bar{v}_{k} x_{r_{1}}+d \sum_{k \in I} v_{k} \otimes v_{\mathbf{i}} \otimes v_{p q} \otimes v_{\mathbf{j}} \otimes \bar{v}_{k}=0
$$

for some $c, d \in \mathbb{C}$. Then $d=c p=c q$ by considering the coefficients of $v_{k} \otimes v_{\mathbf{i}} \otimes v_{p q} \otimes v_{\mathbf{j}} \otimes \bar{v}_{k}$, $k \in\left\{k_{0}, \ell_{0}\right\}$ in the expression of LHS of (6.4). If $c \neq 0$, then $p-q=0$. This is a contradiction since $\lambda_{p q}$ is typical in the sense of (4.4). So, $c=d=0$ and hence $\sum_{k \in I} v_{k} \otimes v_{\mathbf{i}} \otimes v_{p q} \otimes v_{\mathbf{j}} \otimes \bar{v}_{k} x_{r_{1}}$ and $\sum_{k \in I} v_{k} \otimes v_{\mathbf{i}} \otimes v_{p q} \otimes v_{\mathbf{j}} \otimes \bar{v}_{k}$ are linear independent. Now, we assume

$$
\sum_{\mathfrak{t}, d, \kappa_{d}} r_{\mathfrak{t}, d, \kappa_{d}} v_{\lambda, \mathfrak{t}, d, \kappa_{d}}=0 \text { for some } r_{\mathfrak{t}, d, \kappa_{d}} \in \mathbb{C} .
$$

We claim that $r_{\mathfrak{t}, d, \kappa_{d}}=0$ for all possible $\mathfrak{t}, d, \kappa_{d}$. If not, then we pick up a $d \in \mathcal{D}_{r, t}^{f}$ such that

(1) $r_{\mathrm{t}, d, \kappa_{d}} \neq 0$

(2) $d=s_{r-f+1, i_{r-f+1}} \bar{s}_{t-f+1, j_{t-f+1}} \cdots s_{r, i_{r}} \bar{s}_{t, j_{t}}$ and $i_{r}>i_{r-1}>\cdots>i_{r-f+1}$,

(3) $\left(i_{r}, \cdots, i_{r-f+1}\right)$ is maximal with respect to lexicographic order.

Since $r+t \leq \min \{m, n\}$ and $0<f \leq \min \{r, t\}$, we can pick $f$ pairs $\left(k_{i}, \ell_{i}\right), r-f+1 \leq i \leq r$ such that

(1) $k_{i} \in I_{0}, \ell_{i} \in I_{1}, k_{i}>k_{j}$ and $\ell_{i}>\ell_{j}$ if $i>j$;

(2) both $v_{k_{i}}$ and $v_{\ell_{i}}$ are not a tensor factor of $v_{\mathbf{i}_{\mu}}$,

(3) both $\bar{v}_{k_{i}}$ and $\bar{v}_{\ell_{i}}$ are not a tensor factor of $\bar{v}_{\mathbf{j}}$. 
We consider the terms $v_{\mathbf{a}} \otimes v_{p q} \otimes \bar{v}_{\mathbf{b}}$ 's in the expressions of $v_{\lambda, t, d, \kappa_{d}}$ 's in LHS of (6.5) with $r_{\mathrm{t}, d, \kappa_{d}} \neq 0$ such that either $v_{a_{i_{h}}}=v_{k_{h}}$ and $\bar{v}_{b_{i_{t-r+h}}}=\bar{v}_{k_{h}}$ or $v_{a_{i_{h}}}=v_{\ell_{h}}$ and $\bar{v}_{b_{i_{t-r+h}}}=\bar{v}_{\ell_{h}}$ for $r-f+1 \leq h \leq r$. Such terms occur in the expression of $v_{1}^{\otimes f} \otimes \tilde{v}_{\mathfrak{t}} \otimes \bar{v}_{1}^{\otimes f} \mathfrak{e}^{f} d x^{\kappa_{d}}$, where $\tilde{v}_{\mathfrak{t}}$ is a linear combination of the terms in $v_{\mathbf{t}}$ 's (cf. (6.3)) with forms $v_{\mathbf{i}^{\prime}} \otimes v_{p q} \otimes \bar{v}_{\mathbf{j}^{\prime}}$. If $v_{a_{h}}=v_{k_{h}}$ and $\bar{v}_{b_{i_{t-r+h}}}=\bar{v}_{k_{h}}$, by previous arguments, the coefficient of $v_{\mathbf{a}} \otimes v_{p q} \otimes \bar{v}_{\mathbf{b}}$ in $v_{1}^{\otimes f} \otimes v_{\mathfrak{t}} \otimes \bar{v}_{1}^{\otimes f} e^{f} d x^{\kappa_{d}}$ is $\prod_{h=r}^{r-f+1}(-p)^{\epsilon}$, where $\epsilon_{h}=1$ if $\kappa_{h}=1$ and 0 if $\kappa_{h}=0$. If $v_{a_{h}}=v_{\ell_{h}}$ and $\bar{v}_{b_{i_{t-r+h}}}=\bar{v}_{\ell_{h}}$, then the coefficient of $v_{\mathbf{a}} \otimes v_{p q} \otimes \bar{v}_{\mathbf{b}}$ in $v_{1}^{\otimes f} \otimes \tilde{v}_{\mathfrak{t}} \otimes \bar{v}_{1}^{\otimes f} \mathfrak{e}^{f} d x^{\kappa_{d}}$ is $\prod_{h=r}^{r-f+1}(-q)^{\epsilon_{h}}$, where $\epsilon_{h}=1$ if $\kappa_{h}=1$ and 0 if $\kappa_{h}=0$. By (6.5), $\sum_{\mathfrak{t}} r_{\mathfrak{t}, d, \kappa_{d}} \tilde{v}_{\mathfrak{t}}=0$ for any fixed $\kappa_{d}$. Thus, we can assume that $\kappa_{d}=(0, \cdots, 0) \in \mathbf{N}_{f}$. If we identify $\tilde{v}_{\mathfrak{t}}$ with its $v_{p q}$-component, then $\tilde{v}_{\mathfrak{t}}$ can be considered as $\mathfrak{g}_{0}$-highest weight vectors of $V^{\otimes r-f} \otimes W^{\otimes t-f}$ (cf. arguments in the proof of Theorem 5.7) of the form

$$
\tilde{v}_{\mathfrak{t}}=v_{i_{r-f}} \otimes \cdots v_{i_{1}} \otimes \bar{v}_{j_{1}} \otimes \cdots \bar{v}_{j_{t-f}} w_{\mu, \nu} x_{\alpha^{(2)}} y_{\alpha^{(1)}} \bar{y}_{\beta^{(1)}} \bar{x}_{\beta^{(2)}} d(\mathfrak{t}) .
$$

So, $r_{\mathfrak{t}, d, \kappa_{d}}=0$, a contradiction. This proves that $S$ is $\mathbb{C}$-linear independent. Further, $S$ is a basis of $V_{\bar{\lambda}}$ since the cardinality of $S$ is $2^{f}\left|\mathcal{D}_{r, t}^{f}\right| \cdot\left|\mathcal{T}^{s}\left(\mu^{\prime}\right)\right| \cdot\left|\mathcal{T}^{s}\left(\nu^{\prime}\right)\right|$, which is the dimension of space consisting of $\mathfrak{g}_{0}$-highest weight vectors of $V^{r t}$ with highest weight $\mu-\hat{\nu}$.

Definition 6.7. Let $\mathfrak{F}=\operatorname{Hom}_{U(\mathfrak{g})}\left(?, M_{p q}^{r t}\right)$ be the functor from the category of finite dimensional left $\mathfrak{g}$-modules to the category of right $\mathscr{B}_{2, r, t}$-modules over $\mathbb{C}$.

Lemma 6.8. The functor $\mathfrak{F}$ is exact.

Proof. Since $\lambda_{p q}$ is typical, $M^{r t}$ is projective, injective and tilting as left $\mathfrak{g}$-module (e.g., [5, IV]). So, $\mathfrak{F}$ is exact.

Proposition 6.9. Suppose $\lambda=(f, \mu, \nu) \in \Lambda_{2, r, t}$. Then $\mathfrak{F}\left(K_{\bar{\lambda}}\right) \cong C\left(f, \mu^{\prime},\left(\nu^{o}\right)^{\prime}\right)$, where $\nu^{o}=\left(\nu^{(2)}, \nu^{(1)}\right)$.

Proof. By Proposition 3.9, there is an explicit linear isomorphism between $C\left(f, \mu^{\prime},\left(\nu^{o}\right)^{\prime}\right)$ and $V_{\bar{\lambda}}$, where $V_{\bar{\lambda}}$ is given in Theorem 6.6. By Proposition [5.8 and [19, Proposition 6.10], this linear isomorphism is a $\mathscr{B}_{2, r, t}$-homomorphism. Thus, $C\left(f, \mu^{\prime},\left(\nu^{o}\right)^{\prime}\right) \cong V_{\bar{\lambda}}$ as right $\mathscr{B}_{2, r, t^{-}}$ modules. Using the universal property of Kac-modules yields $\operatorname{Hom}_{U(\mathfrak{g})}\left(K_{\bar{\lambda}}, M_{p q}^{r t}\right) \cong V_{\bar{\lambda}}$ as $\mathscr{B}_{2, r, t}-$ modules (cf. the proof of Corollary [5.9). Now, everything is clear.

In the remaining part of this section, we calculate decomposition matrices of $\mathscr{B}_{2, r, t}$. We always assume that $p \in \mathbb{Z}$. Otherwise, one can use $x_{1}+p_{1}$ instead of $x_{1}$ for any $p_{1} \in \mathbb{C}$ with $p-p_{1} \in \mathbb{Z}$. Since $\lambda$ is typical, we have $p-q \notin \mathbb{Z}$ or $p-q \leq-m$ or $p-q \geq n$. In the first case, by [19, Theorem 5.21], $\mathscr{B}_{2, r, t}$ is semisimple and hence its decomposition matrix is the identity matrix. We assume that $p-q \leq-m$. If $p-q \geq n$, one can switch the roles between $p$ and $q$ (or by considering the dual module of $M_{p q}^{r t}$ ) in the following arguments.

Suppose $\lambda=(f, \mu, \nu) \in \Lambda_{2, r, t}$. Let $T_{\bar{\lambda}}$ be the indecomposable tilting module, where $\bar{\lambda}=\lambda_{p q}+\tilde{\lambda}=\lambda_{p q}+\mu-\hat{\nu}$ (cf. Definition 6.2). It is the projective cover of $L_{\bar{\lambda}}$, where $L_{\bar{\lambda}}$ is the simple $\mathfrak{g}$-module with highest weight $\bar{\lambda}$. It is known that $T_{\bar{\lambda}}$ has filtrations of Kacmodules. Let $K_{\bar{\lambda} \text { top }}$ be the unique bottom of $T_{\bar{\lambda}}$. Then $L_{\bar{\lambda}}$ is the simple $\mathfrak{g}$-module of $K_{\bar{\lambda}^{\text {top }}}$. 
Further, $\bar{\lambda}^{\text {top }}$ is the dominant weight defined in Definition 5.11(1). Since $M_{p q}^{r t}$ is a tilting module, it can be decomposed into the direct sum of indecomposable tilting modules

$$
M_{p q}^{r t}=\bigoplus_{\mu \in P^{+}} T_{\mu}^{\oplus \ell_{\mu}} \text { for some } \ell_{\mu} \in \mathbb{N} .
$$

In the remaining part of this paper, we denote $S$ to be the following finite subset of $P^{+}$,

$$
S:=\left\{\mu \in P^{+} \mid \ell_{\mu} \neq 0\right\} .
$$

Parallel to Corollary 5.13, we have the following.

Lemma 6.10. Let $\lambda=(f, \mu, \nu) \in \Lambda_{2, r, t}$ such that $\left(\lambda^{\text {top }}\right)^{\prime}$ is Kleshchev, where $\lambda^{\text {top }}$ is defined in Definition $5.11(2)$. Then $T_{\bar{\lambda}}$ is a direct summand of $M_{p q}^{r t}$.

Proof. We claim that $T_{\bar{\lambda}}$ is a direct summand in $M_{p q}^{r-f, t-f}$. If so, then

$$
v_{1}^{f} \otimes T_{\bar{\lambda}} \otimes \bar{v}_{1}^{f} \mathfrak{e}^{f}
$$

is obviously a tilting submodule in $M_{p q}^{r t}$ which is isomorphic to $T_{\bar{\lambda}}$. Thus the claim implies the result. Therefore, it suffices to consider the case $f=0$.

Denote $\bar{\nu}=\lambda_{p q}-\hat{\nu}$. Since $p \leq q-m$, the weight diagram $D_{\nu}$ (cf. Definition [5.10) of $\bar{\nu}$ is obtained from that of $\lambda_{p q}$ in (5.12) by moving the "> " at vertex $p-i+1$ to its left side at vertex $p-i+1-\nu_{m-i+1}^{(1)}$ for each $i$ with $1 \leq i \leq m$, and moving the " $<$ " at vertex $q-m+j$ to its right side at vertex $q-m+j+\nu_{n-j+1}^{(2)}$ for each $j$ with $1 \leq j \leq n$ (cf. (6.1)). Thus no " $\times$ " can be produced, i.e., $\bar{\nu}$ is typical. Hence $K_{\bar{\nu}}$ is a direct summand in $M_{p q}^{0 t}$. Thus, it suffices to prove that $T_{\bar{\lambda}}$ is a direct summand in $V^{\otimes r} \otimes K_{\bar{\nu}} \ltimes M_{p q}^{r t}$, here $\ltimes$ means direct summand of $M_{p q}^{r t}$. For this, we can apply [5, IV, Lemmas 2.4 and 2.6]. Note from [5. IV, Lemma 2.4] that the action of the functor $F_{i}$ on $K_{\bar{\nu}}$ defined in [5, IV] only depends on symbols at vertices $i$ and $i+1$ of the weight diagram $D_{\nu}$ of $\bar{\nu}$ (we remark that symbols $\circ, \wedge, \vee, \times$ in [5, IV] are respectively symbols $<, \times, \emptyset,>$ in this paper). Due to condition (6.2), for any $i \in I_{p q}:=I_{p q}^{+} \backslash\{q-m+n\}$ such that $i$ is involved in a path in the crystal graph in [5. IV, Lemma 2.6], the symbols at vertex $i$ and $i+1$ in the weight diagram $D_{\nu}$ of $\bar{\nu}$ are the same as that in the weight diagram $D_{\emptyset}$ of $\lambda_{p q}$. This shows that $T_{\bar{\lambda}}$ is a direct summand in $V^{\otimes r} \otimes K_{\bar{\nu}}$ if and only if $T_{\lambda_{p q}+\tilde{\mu}}$ is a direct summand in $V^{\otimes r} \otimes K_{\lambda_{p q}}$, more precisely, [5, IV, Lemma 2.6] implies

$$
F_{i_{r}} \cdots F_{i_{1}} K_{\bar{\nu}} \cong T_{\bar{\lambda}}^{\otimes 2^{\ell}} \Longleftrightarrow F_{i_{r}} \cdots F_{i_{1}} K_{\lambda_{p q}} \cong T_{\lambda_{p q}+\tilde{\mu}}^{\otimes 2^{\ell}},
$$

where $\ell$ is the number of edges in the given path of the form $\emptyset \times \rightarrow<>$. Thus the result follows from Corollary 5.13 .

We remark that there is a bijection between $S$ defined in (6.7) and the set of pair-wise nonisomorphic simple $\mathscr{B}_{2, r, t}$-modules. See [19, Theorem 7.5]. For any $\xi \in S$ as above, parallel to Definition 5.11, we define $\xi^{\text {top }}$ to be the unique dominant weight such that $L_{\xi}$ is the simple submodule of $K_{\xi^{\text {top }}}$.

Proposition 6.11. For any $\xi \in S$, there is a unique $(f, \mu, \nu) \in \Lambda_{2, r, t}$ such that $\xi^{\text {top }}=\lambda_{p q}+\mu-\hat{\nu}$. Further, $\mathfrak{F}\left(T_{\xi}\right)$ is isomorphic to the projective cover of $D^{f, \mu^{\prime},\left(\nu^{0}\right)^{\prime}}$, where $D^{f, \mu^{\prime},\left(\nu^{0}\right)^{\prime}}$ is the simple head of $C\left(f, \mu^{\prime},\left(\nu^{o}\right)^{\prime}\right)$. 
Proof. If $\xi \in S$, then $T_{\xi}$ is an indecomposable tilting module with $\ell_{\xi}>0$. By Theorem 4.4, $\mathfrak{F}\left(T_{\xi}\right)$ is a direct sum of certain principle indecomposable right $\mathscr{B}_{2, r, t}$-modules. We claim that $\mathfrak{F}\left(T_{\xi}\right)$ is indecomposable for any $\xi \in S$. Otherwise, $\sum_{\xi \in S} \ell_{\xi}$ is strictly less than the number of principal indecomposable direct summands of right $\mathscr{B}_{2, r, t}$-module $\mathscr{B}_{2, r, t}$. However, for each principal indecomposable direct summand $P$ of left $\mathscr{B}_{2, r, t}$-module $\mathscr{B}_{2, r, t}, P$ has to be a projective cover of irreducible left $\mathscr{B}_{2, r, t}$-module, say $D$, which is the simple head of a left cell module, say $\Delta(\ell, \alpha, \beta)$ for some $(\ell, \alpha, \beta) \in \Lambda_{2, r, t}$, where $\Delta(\ell, \alpha, \beta)$ is defined via a weakly cellular basis of $\mathscr{B}_{2, r, t}$. So, there is an epimorphism from $P$ to $\Delta(\ell, \alpha, \beta)$. Since $\mathfrak{G}:=M_{p q}^{r t} \otimes_{\mathscr{B}_{2, r, t}}$ ? is right exact, there is an epimorphism from $\mathfrak{G}(P)$ to $\mathfrak{G}(\Delta(\ell, \alpha, \beta))$. If $\mathfrak{G}(\Delta(\ell, \alpha, \beta)) \neq 0$, then $\mathfrak{G}(P)$ is a non-zero direct summand of $M_{p q}^{r t}$. This implies that the number of indecomposable direct summands of left $\mathscr{B}_{2, r, t}$-module $\mathscr{B}_{2, r, t}$ is strictly less than $\sum_{\xi \in S} \ell_{\xi}$. This is a contradiction since the number of principal indecomposable direct summands of left $\mathscr{B}_{2, r, t}$-module $\mathscr{B}_{2, r, t}$ is equal to that of right $\mathscr{B}_{2, r, t}$-module $\mathscr{B}_{2, r, t}$. So, $\mathfrak{F}\left(T_{\xi}\right)$ is indecomposable. Since $K_{\xi^{\text {top }}} \hookrightarrow T_{\xi}$, we have $\mathfrak{F}\left(T_{\xi}\right) \rightarrow \mathfrak{F}\left(K_{\xi^{\text {top }}}\right)$. By Proposition 6.9. $\mathfrak{F}\left(K_{\xi_{\text {top }}}\right) \cong C\left(f, \mu^{\prime},\left(\nu^{o}\right)^{\prime}\right)$. Thus, $C\left(f, \mu^{\prime},\left(\nu^{o}\right)^{\prime}\right)$ has the simple head, denoted by $D^{f, \mu^{\prime},\left(\nu^{o}\right)^{\prime}}$, and hence $\mathfrak{F}\left(T_{\xi}\right)=P^{f, \mu^{\prime},\left(\nu^{o}\right)^{\prime}}$. Since $\xi \in S$, by Lemma 6.10, both $\mu^{\prime}$ and $\left(\nu^{o}\right)^{\prime}$ are Kleshchev in the sense of (3.15) with respect to $-p, m-q$ and $q, p-n$.

It remains to prove $\mathfrak{G}\left(\Delta\left(\ell, \alpha, \beta^{o}\right)\right) \neq 0$ for any $\delta:=(\ell, \alpha, \beta) \in \Lambda_{2, r, t}$. By Theorem 6.6. $V_{\bar{\delta}}$ contains a non-zero vector $v:=v_{1}^{\otimes \ell} \otimes v_{\mathbf{i}} \otimes v_{p q} \otimes v_{\mathbf{j}} \otimes \bar{v}_{1}^{\otimes \ell} \mathfrak{e}^{f} w_{\alpha, \beta} \mathfrak{y}_{\alpha^{\prime}} \overline{\mathfrak{y}}_{\left(\beta^{o}\right)^{\prime}}$, where $\mathbf{i}$ and $\mathbf{j}$ are defined as in Definition 6.4. So, it is enough to show $v \in \mathfrak{G}\left(\Delta\left(\ell, \alpha, \beta^{o}\right)\right)$, where $\Delta\left(\ell, \alpha, \beta^{o}\right)$ is defined via a suitable weakly cellular basis of $\mathscr{B}_{2, r, t}$. We use cellular bases of $\mathscr{H}_{2, r-f}$ and $\mathscr{H}_{2, t-f}$ in Lemma 3.3 (1) (3) to construct a weakly cellular basis of $\mathscr{B}_{2, r, t}$, which is similar to that in Theorem 3.6. Let $\Delta\left(\ell, \alpha, \beta^{\circ}\right)$ be the corresponding left cell module with respect to $\left(\ell, \alpha, \beta^{o}\right) \in \Lambda_{2, r, t}$. By arguments similar to those for the proof of Proposition [3.9, one can verify

$$
\Delta\left(\ell, \alpha, \beta^{o}\right) \cong \mathscr{B}_{2, r, t} \mathfrak{e}^{f} \mathfrak{x}_{\alpha} \overline{\mathfrak{x}}_{\beta^{o}} w_{\alpha, \beta} \mathfrak{y}_{\alpha^{\prime}} \overline{\mathfrak{y}}_{\left(\beta^{o}\right)^{\prime}} \quad\left(\bmod \mathscr{B}_{2, r, t}^{\ell+1}\right) .
$$

Let $M=\tilde{v} \mathscr{B}_{2, r, t}$ be the cyclic $\mathscr{B}_{2, r, t}$-module generated by $\tilde{v}:=v_{1}^{\otimes \ell} \otimes v_{\mathbf{i}} \otimes v_{p q} \otimes v_{\mathbf{j}} \otimes \bar{v}_{1}^{\otimes \ell}$. Then $M \otimes \mathscr{B}_{2, r, t} \Delta\left(\ell, \alpha, \beta^{o}\right)$ is a subspace of $\mathfrak{G}\left(\Delta\left(\ell, \alpha, \beta^{o}\right)\right)$. Since $\mathscr{B}_{2, r, t}^{\ell+1}$ acts on $M$ trivially, there is a $\mathbb{C}$-linear map $\phi: M \otimes_{\mathscr{B}_{2, r, t}} \Delta\left(\ell, \alpha, \beta^{o}\right) \rightarrow M$ such that $\phi(m \otimes \bar{h})=m h$ for any $\bar{h} \in \mathscr{B}_{2, r, t} \mathfrak{e}^{f} \mathfrak{x}_{\alpha} \overline{\mathfrak{x}}_{\beta^{o}} w_{\alpha, \beta} \mathfrak{y}_{\alpha^{\prime}} \overline{\mathfrak{y}}_{\left(\beta^{\circ}\right)^{\prime}}\left(\bmod \mathscr{B}_{2, r, t}^{\ell+1}\right)$. Since $\lambda_{p q}$ is typical and the ground filed is $\mathbb{C}$, up to a non-zero scalar, we have $v=\phi(\tilde{v} \otimes \bar{h})$, where $h \equiv \mathfrak{e}^{f} w_{\alpha, \beta} \mathfrak{y}_{\alpha^{\prime}} \overline{\mathfrak{y}}_{\left(\beta^{o}\right)^{\prime}}\left(\bmod \mathscr{B}_{2, r, t}^{\ell+1}\right)$. Thus, $\mathfrak{G}\left(\Delta\left(\ell, \alpha, \beta^{o}\right)\right) \neq 0$.

Remark 6.12. Proposition 6.11 implies that $C(f, \mu, \nu)$ has the simple head if $\mu$ and $\nu$ are Kleshchev bipartitions with respect to $-p, m-q$ and $q, p-n$ in the sense of (3.15). Further, all non-isomorphic simple $\mathscr{B}_{2, r, t}$-modules can be realized in this way.

Proposition 6.13. Suppose $\xi \in P^{+}$. Then $\mathfrak{F}\left(L_{\xi}\right)=0$ if $\xi \notin S($ cf. (6.7)) and $\mathfrak{F}\left(L_{\xi}\right) \cong D^{f, \mu^{\prime},\left(\nu^{o}\right)^{\prime}}$ if $\xi \in S$, where $\xi^{\text {top }}=\lambda_{p q}+\mu-\hat{\nu}$ with $(f, \mu, \nu) \in \Lambda_{2, r, t}$.

Proof. By (6.6), $\mathfrak{F}\left(L_{\xi}\right)=\oplus_{\zeta \in S} \operatorname{Hom}_{\mathfrak{g}}\left(L_{\xi}, T_{\zeta}^{\otimes \ell_{\zeta}}\right)$. Suppose $0 \neq f \in \operatorname{Hom}_{U(\mathfrak{g})}\left(L_{\xi}, T_{\zeta}^{\oplus \ell_{\zeta}}\right)$. Then $L_{\xi} \cong f\left(L_{\xi}\right)$ is a simple submodule of $T_{\zeta}^{\oplus \ell_{\zeta}}$. Since $T_{\zeta}$ has the unique simple submodule $L_{\zeta}$, 
$\mathfrak{F}\left(L_{\xi}\right)=0$ if $\xi \notin S$. If $\xi \in S$, then

$$
\mathfrak{F}\left(L_{\xi}\right)=\operatorname{Hom}_{U(\mathfrak{g})}\left(L_{\xi}, T_{\xi}^{\oplus \ell_{\xi}}\right),
$$

which is obviously $\ell_{\xi}$-dimensional. Let $v_{\xi}^{1}, \ldots, v_{\xi}^{\ell_{\xi}} \in T_{\xi}^{\oplus \ell_{\xi}}$ be the generators of the tilting module $T_{\xi}^{\oplus \ell_{\xi}}$ (then $v_{\xi}^{1}, \ldots, v_{\xi}^{\ell_{\xi}}$ span the generating space, denoted $\boldsymbol{V}$, of $T_{\xi}^{\oplus \ell_{\xi}}$ ), and $v_{\xi}^{\prime 1}, \ldots, v_{\xi}^{\prime \ell_{\xi}} \in L_{\xi}^{\oplus \ell_{\xi}}$, the corresponding generators of the submodule $L_{\xi}^{\oplus \ell_{\xi}}$ of $T_{\xi}^{\oplus \ell_{\xi}}$. Thus, there exists a unique $u \in U(\mathfrak{g})$ such that

$$
v_{\xi}^{\prime i}=u v_{\xi}^{i} \text { for } i=1, \ldots, \ell_{\xi} .
$$

Let $\tilde{v}_{\xi} \in L_{\xi}$ be the generator of the simple module $L_{\xi}$. As in the proof of Corollary 5.14, we can define $f^{i}: L_{\xi} \rightarrow T_{\xi}^{\oplus \ell_{\xi}}$ to be the $U(\mathfrak{g})$-homomorphism sending $\tilde{v}_{\xi}$ to $v_{\xi}^{\prime i}$ for $i=1, \ldots, \ell_{\xi}$. Then $\left(f^{1}, \ldots, f^{\ell_{\xi}}\right)$ is obviously a basis of $\mathfrak{F}\left(L_{\xi}\right)$ (cf. (6.8) $)$.

For any $A \in M_{\ell_{\xi}}$ (the algebra of $\ell_{\xi} \times \ell_{\xi}$ complex matrices), we can define an element $\phi_{A} \in \operatorname{End}_{U(\mathfrak{g})}\left(M_{p q}^{r t}\right)=\mathscr{B}_{2, r, t}$ as follows: $\left.\phi_{A}\right|_{T_{\zeta}^{\oplus \ell_{\zeta}}}=0$ if $\zeta \neq \xi$ and

$$
\left.\phi_{A}\right|_{T_{\xi}^{\oplus \ell_{\xi}}}:\left(v_{\xi}^{1}, \ldots, v_{\xi}^{\ell_{\xi}}\right) \mapsto\left(v_{\xi}^{1}, \ldots, v_{\xi}^{\ell_{\xi}}\right) A \text { (regarded as vector-matrix multiplication), }
$$

i.e., the transition matrix of the action of $\left.\phi_{A}\right|_{T_{\xi}^{\oplus \ell_{\xi}}}$ on the generating space $\boldsymbol{V}$ of $T_{\xi}^{\oplus \ell_{\xi}}$ under the basis $\left(v_{\xi}^{1}, \ldots, v_{\xi}^{\ell_{\xi}}\right)$ is $A$. By the universal property of projective modules, this uniquely defines an element $\phi_{A} \in \mathscr{B}_{2, r, t}$. Thus we have the embedding $\phi: M_{\ell_{\xi}} \rightarrow \mathscr{B}_{2, r, t}$ sending $A$ to $\phi_{A}$. Write $A$ as $A=\left(a_{i j}\right)_{i, j=1}^{\ell_{\xi}}$. Then by (6.10) and definition of the right action of $\mathscr{B}_{2, r, t}$ on $M_{p q}^{r t}$, we have

$$
f^{i}\left(\tilde{v}_{\xi}\right) \phi_{A}=v_{\xi}^{\prime i} \phi_{A}=\left(u v_{\xi}^{i}\right) \phi_{A}=u\left(v_{\xi}^{i} \phi_{A}\right)=u \sum_{j=1}^{\ell_{\xi}} a_{j i} v_{\xi}^{j}=\sum_{j=1}^{\ell_{\xi}} a_{j i} v_{\xi}^{\prime j}=\left(\sum_{j=1}^{\ell_{\xi}} a_{j i} f^{j}\right)\left(\tilde{v}_{\xi}\right),
$$

i.e., the transition matrix of the action of $\phi_{A}$ on $\mathfrak{F}\left(L_{\xi}\right)$ under the basis $\left(f^{1}, \ldots, f^{\ell_{\xi}}\right)$ is $A$. Thus $\phi\left(M_{\ell_{\xi}}\right)$ acts transitively on the $\ell_{\xi}$-dimensional space $\mathfrak{F}\left(L_{\xi}\right)$ and hence $\mathfrak{F}\left(L_{\xi}\right)$ is a simple $\mathscr{B}_{2, r, t}$-module. Finally, since $L_{\xi} \hookrightarrow K_{\xi^{\text {top }}}$, we have $\mathfrak{F}\left(K_{\xi^{\text {top }}}\right) \rightarrow \mathfrak{F}\left(L_{\xi}\right)$. Note that $D^{f, \mu^{\prime},\left(\nu^{o}\right)^{\prime}}$ is the simple head of $\mathfrak{F}\left(K_{\xi^{\text {top }}}\right)$. Thus, $\mathfrak{F}\left(L_{\xi}\right) \cong D^{f, \mu^{\prime},\left(\nu^{o}\right)^{\prime}}$.

Theorem 6.14. Suppose $(f, \alpha, \beta) \in \Lambda_{2, r, t}$ such that there is a $\lambda \in S$ (cf. (6.7)) satisfying $\lambda^{\text {top }}=\lambda_{p q}+\alpha-\hat{\beta}$. If $\mu:=(\ell, \gamma, \delta) \in \Lambda_{2, r, t}$, then $\left[C\left(\ell, \gamma^{\prime},\left(\delta^{o}\right)^{\prime}\right): D^{\left.f, \alpha^{\prime},\left(\beta^{o}\right)^{\prime}\right]}=\left(T_{\lambda}: K_{\bar{\mu}}\right)\right.$.

Proof. The result follows from Lemma 6.8, Propositions 6.9 and 6.13, together with the BGG reciprocity formula for $\mathfrak{g}$.

\section{REFERENCES}

[1] S. Ariki and A. Mathas, "The number of simple modules of the Hecke algebras of type $G(r, 1, n)$ ", Math. Z. 233 (2000), 601-623.

[2] S. Ariki and A. Mathas, "On the classification of simple modules for cyclotomic Hecke algebras of type $G(m, 1, n)$ and Kleshchev multipartitions", Osaka J. Math. 38 (2001), 827-837.

[3] S. Ariki, A. Mathas and H. Rui, "Cyclotomic Nazarov-Wenzl algebras", Nagoya Math. J., Special issue in honor of Prof. G. Lusztig's sixty birthday, 182 (2006), 47-134.

[4] J. BRundAn, "Kazhdan-Lusztig polynomials and character formulae for the Lie superalgebra $\mathfrak{g l}_{m \mid n} "$, J. Amer. Math. Soc. 16 (2002), 185-231. 
[5] J. BRUNDAN and C. Stroppel, "Highest weight categories arising from Khovanov's diagram algebra I, II, III, IV", Moscow Math. J. 11, (2011), 685-722; Transform. Groups 15, (2010), 1-45; Represent. Theory 15, (2011), 170-243; J. Eur. Math. Soc. 14, (2012), 373-419.

[6] J. Brundan and C. Stroppel, "Gradings on walled Brauer algebras and Khovanov's arc algebra, Adv. Math. 231 (2012), no. 2, 709-773.

[7] R. Dipper and G. G. James "Representations of Hecke algebras of type $B_{n}$ ", J. Algebra 146 (1992), 454-481.

[8] R. Dipper and G. James and E. Murphy "Hecke algebras of type $B_{n}$ at roots of unity", Proc. London Math. Soc. (3) 70 (1995), no. 3, 505-528.

[9] R. Dipper and A. Mathas, "Morita equivalences of Ariki-Koike algebras", Math. Z. 240 (2002), $579-610$

[10] V.G. Drinfeld, "Degenerate affine Hecke algebras and Yangians", Func. Anal. Appl., 20 (1986), $62-64$.

[11] F. Goodman and J. Graber, "Cellularity and the Jones basic construction ", Adv. in Appl. Math. 46 (2011), 312-362

[12] J. J. Graham and G. I. Lehrer, "Cellular algebras", Invent. Math. 123 (1996), 1-34.

[13] C. Groson and V. Serganova, "Cohomology of generalized supergrassmannians and character formulae for basic classical Lie superalgebras", Proc. London Math. Soc. 101 (2010), 852-892.

[14] V.G. KaC, "Lie superalgebras", Adv. Math. 26 (1977), 8-96.

[15] A. Kleshchev, "Linear and projective representations of symmetric groups", Cambridge Tracts in Mathematics, 163. Cambridge University Press, Cambridge, 2005.

[16] K. KoIke, "On the decomposition of tensor products of the representations of classical groups: By means of universal characters", Adv. Math. 74 (1989) 57-86.

[17] P. Nikitin, "The centralizer algebra of the diagonal action of the group GLn(C) in a mixed tensor space", J. Math. Sci. 141 (2007), 1479-1493.

[18] H. Rui and L. Song, "Decomposition numbers of quantized walled Brauer algebras", preprint, 2011.

[19] H. Rui and Y. Su, "Affine walled Brauer algebras and super Schur-Weyl duality", arXiv:1305.0450.

[20] A. SARTORI, "The degenerate affine walled Brauer algebra", arXiv: 1305.2347

[21] Y. Su, J.W.B. Hughes and R.C. KIng, "Primitive vectors in the Kac-module of the Lie superalgebra $\operatorname{sl}(m \mid n) "$, J. Math. Phys. 41 (2000), 5044-5087.

[22] Y. Su and R.B. Zhang, "Generalised Jantzen filtration of Lie superalgebras I", J. Eur. Math. Soc. 14 (2012), 1103-1133.

[23] V. Turaev, "Operator invariants of tangles and R-matrices", Izv. Akad. Nauk SSSR Ser. Math. 53 (1989) 1073-1107 (in Russian).

[24] J. van DER JEugt and R.B. Zhang, "Characters and composition factor multiplicities for the Lie superalgebra $g l(m \mid n) "$, Lett. Math. Phys. 47 (1999), 49-61.

[25] M. VAzIRANi, "Parameterizing Hecke algebra modules: Bernstein-Zelevinsky multisegments, Kleshchev multipartitions, and crystal graph", Transformation Groups 7 (2002), no. 3, 267-303.

H. Rui: Department of Mathematics, Shanghai Key Laboratory of PMMP, East China Normal University, Shanghai, 200241, China

E-mail address: hbrui@math.ecnu.edu.cn

Y. Su: Department of Mathematics, Tongui University, Shanghai, 200092, China

E-mail address: ycsu@tongji.edu.cn 